\title{
Wirkung von Forschungsausgaben
}

\section{Christian KEUSCHNIGG}

Wirtschaftspolitisches Zentrum WPZ, Universität St. Gallen (FGN-HSG)

Gerald GOGOLA, Julian JOHS, Mara KRITZINGER und Sascha SARDADVAR

WPZ Research, Wien

Studie im Auftrag des Bundesministeriums für Digitalisierung und Wirtschaftsstandort 


\title{
Wirkung von Forschungsausgaben
}

\author{
Christian KEUSCHNIGG \\ Professor für Nationalökonomie und Leiter des Wirtschaftspolitischen Zentrums \\ WPZ der Universität St. Gallen (FGN-HSG). \\ Kontakt: Christian.Keuschnigg@unisg.ch
}

\section{Gerald GOGOLA, Julian JOHS, Mara KRITZINGER und Sascha SARDADVAR}

Wissenschaftliche Mitarbeiterinnen und Mitarbeiter des WPZ Research in Wien

31. Mai 2021

\section{In Kürze:}

Wie groß sind die Wirkungen der Ausgaben für Forschung und Entwicklung (F\&E)? Mit F\&E bauen die Unternehmen ihre Wettbewerbsfähigkeit aus und sichern die künftigen Gewinne. Die Forschungsförderung regt die F\&E-Investitionen an und verhilft innovativen Unternehmen zu mehr Wachstum. Mit ökonometrischen Matching-Methoden vergleichen wir die Entwicklung von Unternehmen, die durch die FFG gefördert wurden, relativ zu nicht-geförderten, aber sonst ganz ähnlichen Unternehmen. Unternehmen, die eine FFG-Förderung erhalten, konnten im Zeitraum 2016-2019 ihre Beschäftigung um 7,1\% steigern, andere vergleichbare Unternehmen nur um 0,4\%. Das Umsatzwachstum beträgt 10,8\% statt 2,5\%. Mit einem detaillierten Innovationsmodell der österreichischen Wirtschaft berechnen wir die makroökonomischen Effekte von F\&E-Ausgaben. In einem Wachstumsszenario nach dem Muster der letzten zehn Jahre lassen sich etwa $28 \%$ des Wachstums ursächlich auf die Wirkungen des Innovationssystems zurückführen. Dieser Effekt wird mit F\&E-Ausgaben von nur 3,18\% des BIPs erzielt. Eine Erhöhung der öffentlichen F\&E-Ausgaben um 1 Euro könnte langfristig das BIP um bis zu 6 Euro erhöhen. Allerdings haben F\&E-Ausgaben lange Vorlaufzeiten, so dass die positiven Wirkungen nur allmählich eintreten.

Studie im Auftrag des Bundesministeriums für Digitalisierung und Wirtschaftsstandort ${ }^{1}$

\footnotetext{
Wir danken dem Bundesministerium für Digitalisierung und Wirtschaftsstandort für die Beauftragung der Studie, dem Begleitteam für viele wertvolle Kommentare und der FFG für die Bereitstellung der Förderdaten und die hilfreichen Anregungen.
} 


\section{Inhalt}

Inhalt

Kurzzusammenfassung ............................................................................................................................ II

Executive Summary ………………………………………….................................................................

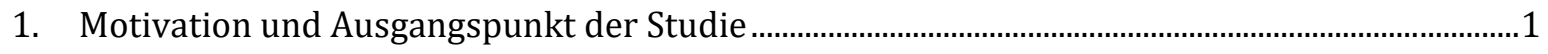

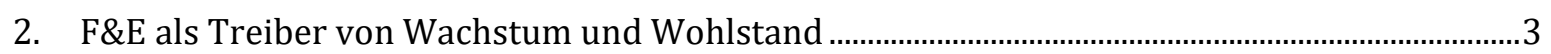

$2.1 \quad$ Theoretische Fundierung .....................................................................................................

$2.2 \quad$ Empirische Ergebnisse rezenter Studien................................................................................

3. Wirtschaftspolitische Rahmenbedingungen ..................................................................................... 10

3.1 Entwicklungen und FTI-Ziele auf europäischer Ebene........................................................... 10

3.2 Entwicklungen und FTI-Ziele auf nationaler Ebene................................................................. 15

4. Wirkungen von Forschungsausgaben auf Makroebene ..................................................................... 21

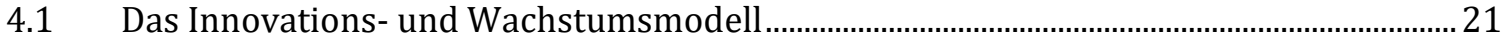

4.2 Langfristige Wirkungen öffentlicher Forschungsausgaben .................................................... 23

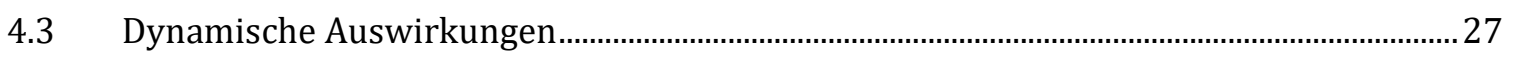

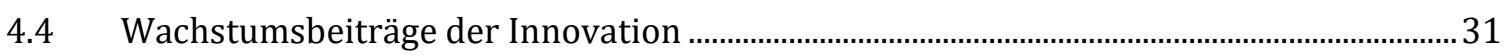

5. Wirkungen von Forschungsausgaben auf Mikroebene ....................................................................36

$5.1 \quad$ Datenbasis ...........................................................................................................................

5.2 FFG Förderung und Unternehmensentwicklung.................................................................. 40

5.3 Die Charakteristika geförderter Unternehmen ................................................................... 44

5.4 F\&E-Ausgaben, FFG-Förderung und Unternehmenserfolg ..................................................... 48

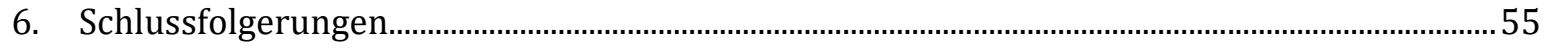

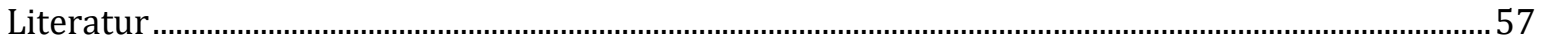

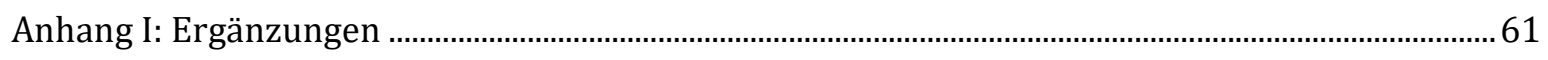

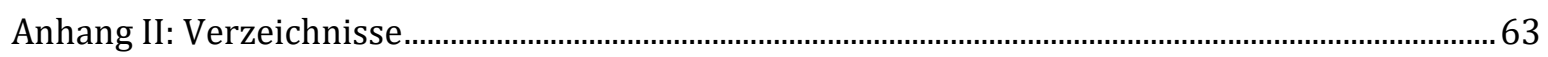




\section{Kurzzusammenfassung}

Wie groß sind die Wirkungen der öffentlichen F\&E-Ausgaben? Der Einsatz des WPZ Innovationsmodells liefert für die makroökonomischen Auswirkungen folgende Ergebnisse: Ein Euro an öffentlichen Mehrausgaben für die Forschung, Entwicklung und Innovation führt zu einem langfristigen BIP-Zuwachs von 6 Euro. In einem Wachstumsszenario nach dem Muster der vergangenen zehn Jahre sind daher etwa $28 \%$ des kumulativen Wachstums auf die Effekte des heimischen Innovationssystems zurückzuführen. Mit öffentlichen Forschungsausgaben, also den Ausgaben für öffentliche Grundlagenforschung, für die Forschungsprämie und für die direkten Förderungen der FFG, kann der Staat wichtige Impulse für mehr private F\&E-Investitionen setzen. Damit hebt er die Fähigkeit des Innovationssystems, Wohlstand zu schaffen und neue Lösungen für die großen gesellschaftlichen Herausforderungen wie Digitalisierung, Klimakrise oder Gesundheitskrise zu finden.

Die Unternehmen investieren in F\&E, weil sie ihre Wettbewerbsfähigkeit ausbauen, Marktanteile gewinnen und so die künftigen Gewinne steigern wollen. Um die Auswirkungen auf Unternehmensebene festzumachen, vergleichen wir mit ökonometrischen Methoden die Entwicklung von FFG-geförderten Unternehmen relativ zu nicht-geförderten, aber sonst ganz ähnlichen Unternehmen. Das Ergebnis ist, dass die FFG-geförderten Unternehmen ihre Beschäftigung im Zeitraum 2016-2019 um 7,1\% steigern konnten, andere vergleichbare Unternehmen dagegen nur um 0,4 \%. Das Umsatzwachstum beträgt 10,8 \% statt 2,5\%. Auch in anderen Dimensionen des Unternehmenserfolgs sind F\&E-intensive Unternehmen den Konkurrenten deutlich überlegen.

F\&E-Ausgaben haben eine außerordentlich hohe volkswirtschaftliche Rendite, welche die private Rendite der Unternehmen bei weitem übersteigt. Die Investitionen der einzelnen Unternehmen zeitigen eine zusätzliche Rendite für die gesamte Volkswirtschaft. Die F\&E-Anstrengungen eines Unternehmens bauen auf den Erfahrungen und Erkenntnissen der anderen auf. In der Innovation ist daher das Ganze mehr als die Summe der Teile. Um die Überschussrendite der privaten Innovation zu realisieren, sollte der Staat mit steuerlichen Anreizen und direkten Subventionen mehr private F\&E-Investitionen anstoßen (Korrektur eines Marktversagens). Die Ausgaben für öffentliche Grundlagenforschung sind eine zentrale Vorleistung für private Innovationen. Wie bei anderen öffentlichen Gütern muss der Staat den Löwenanteil finanzieren, um die Voraussetzungen für erfolgreiche private Innovation zu schaffen. Ein investiver Staat muss die Budgetmittel dorthin lenken, wo sie für die Gesellschaft den höchsten Ertrag erwirtschaften. Wie die Ergebnisse der Studie zeigen, kann der Staat mit Mehrausgaben für F\&E die Voraussetzungen für nachhaltiges Wachstum wesentlich verbessern.

Angesichts großer gesellschaftlicher und externer Herausforderungen wie der Klimakrise oder der digitalen Transformation gilt es, sowohl die Grundlagenforschung auszubauen als auch strategische Prioritäten in der FTI-Politik zu setzen, damit das heimische Innovationssystem zur Bewältigung und Lösung dieser Herausforderungen beitragen und damit Wachstum und künftigen Wohlstand sichern kann. Eine besondere Situation schafft die aktuelle COVID-19Pandemie. Es ist absehbar, dass die F\&E-Ausgaben des Unternehmenssektors, der für knapp die Hälfte $(47,6 \%)$ der österreichischen F\&E-Ausgaben verantwortlich ist, drastisch zurückgehen werden. Umso kritischer ist es, dass der öffentliche Sektor durch langfristige öffentliche Finanzierung einen Rückfall in der Innovationsperformance verhindert. Einsparungen bei den öffentlichen Forschungsausgaben im Zuge des Abbaus der COVID-19-Schulden würde der Wettbewerbsfähigkeit der Wirtschaft und dem Wachstumspotential Österreichs nachhaltig schaden, das Aufholen nach der Krise wesentlich verzögern, und damit die Budgetkonsolidierung zusätzlich erschweren. 
Die Innovationspolitik braucht allerdings einen langen Atem. Zwar steigen die Wachstumsraten relativ rasch an, aber es braucht viel Zeit, bis die kumulativen Effekte auf den mit F\&E geschaffenen Wissensbestand und damit die möglichen Produktivitätssteigerungen vollständig eintreten. Das ist für die Wirtschaftspolitik eine Herausforderung. Die höheren F\&E-Ausgaben sind sofort da, die positiven volkswirtschaftlichen Wirkungen treten dagegen nur langsam ein. Auch im Unternehmenssektor haben F\&E-Investitionen eine lange Vorlaufzeit. Es dauert, bis die höheren Gewinne fließen. In der Grundlagenforschung sind die Vorlaufzeiten noch viel länger. Deshalb gilt in der Innovationspolitik: Wer Technologieführerschaft erreichen will, muss zuerst investieren. 


\section{Executive Summary}

How large are the effects of public R\&D spending? Using the WPZ innovation model for Austria, we evaluate the macroeconomic effects of public R\&D spending and report the following results: One Euro of additional public R\&D spending yields long-run GDP gains of about 6 Euros. In a growth scenario that captures the stylized facts of the past ten years, we compute that about $28 \%$ of cumulative growth is due to the impact of the innovation system. Our findings imply that public research spending, including spending on basic research and the budget costs of tax credits ("Forschungsprämie") and direct R\&D subsidies (FFG funds), provides powerful stimuli for more private R\&D investment. The government thereby raises the capacity of the innovation system to generate growth and welfare and to find new solutions to current challenges such as digital transformation, climate crisis and health problems.

Private companies invest in R\&D to improve their competitive position, to capture additional market shares, and to boost future profits. To quantify the effects of private R\&D at the firm level, we use econometric matching techniques and compare the performance of firms with FFG research grants relative to other, comparable firms that receive no such subsidies. The result is that firms which attracted competitive R\&D grants in 2016, were able to expand employment by $7.1 \%$ in the subsequent period of 2016-2019. In contrast, other comparable firms without such grants were able to increase employment by merely $0.4 \%$. Firms with R\&D grants were able to boost sales by $10.8 \%$ over the same period, compared to $2.5 \%$ by other firms. Innovative firms also outperform their rivals in other dimensions of company performance.

R\&D spending yields a high social return to investment, much higher than the return recorded by private innovators. The R\&D investments of individual companies yield an additional "external return" that allows other firms to build on the results and experiences of the innovator and to be more successful in their own R\&D. The social value created by innovation is more than the sum of the individual parts. Since external returns are non-rival and not excludable, firms cannot get compensated for that part of the social return and therefore tend to invest too little in R\&D. To allow society to reap the full social returns to innovation, governments typically stimulate more R\&D activity by using tax credits, direct R\&D subsidies and by investing in basic public research. Basic research is a critical input into private R\&D. As with other public goods, the government must finance the lion's share of basic research and thereby improves the preconditions for successful private R\&D. The government should allocate scarce tax money to those uses where the social returns are highest. The findings of the study imply that the government could substantially improve Austria's potential for more growth and welfare by raising R\&D expenditure.

In confronting the great current challenges to society such as climate change, digitization and health problems, public research policy must strengthen basic research as well as set strategic priorities. It thereby improves the potential of the innovation system to develop new solutions to current challenges and to promote growth and welfare. The COVID-19-pandemic creates a special problem. Given the procyclical nature of R\&D spending, one must expect that private R\&D, which accounts for almost half (47.6 \%) of total Austrian R\&D spending, will take a severe hit as a result of the current recession. It is thus even more critical that the public sector sets a reliable growth path for public research spending to prevent lasting damage to national innovation performance. Although the government will be forced to consolidate the large additional COVID-19 debt, budget cuts on R\&D spending would be counterproductive. They would impair competitiveness and growth of the Austrian economy, thereby much delay the recovery from the COVID recession, and make budget consolidation even more difficult. 
Innovation policy requires patience. When allocating additional resources for more R\&D, growth rates increase relatively fast, but it takes a long time until the cumulative effects on knowledge stocks and knowhow fully materialize. In consequence, productivity gains set in with rather slow speed. Such a pattern creates a policy challenge. While the budget costs of higher public R\&D spending are immediate, the income gains created by induced innovation set in very slowly. However, the problem is no different from the need to pre-finance private R\&D while additional profits arrive only with substantial delay. The gestation period of basic research is even longer. The conclusion for innovation policy is: before achieving technology leadership, one must first invest. 


\section{Motivation und Ausgangspunkt der Studie}

F\&E wird - neben Bildung - als zentrale Voraussetzung für Wachstum und Wohlstand angesehen. Die Gesellschaft soll so weit als möglich Nutzen aus dem technischen Fortschritt (wie z.B. der Digitalisierung) ziehen, damit sie die großen Herausforderungen wie Klimakrise oder COVID-19Pandemie mit innovativen Lösungen besser bewältigen kann. Der verstärkte Fokus auf die Entwicklung und Anwendung von partizipativen Ansätzen, wie Open Science, Citizen Science und CoCreation, in den vergangenen Jahren soll zudem den Erfolg von F\&E steigern und damit einen höheren Impact mit mehr (Breiten-)Wirkung erzielen. Österreich zählt zu jenen Ländern, die sehr hohe Summen an öffentlichen und privaten Mitteln für F\&E aufbringen. Im den vergangenen zwei Jahrzehnten sind die F\&E-Aufwendungen in Österreich stark gestiegen. Die F\&E-Quote, die 1999 noch 1,85 \% (F\&E-Ausgaben von 3,76 Mrd. €) betrug, lag 2019 bereits bei 3,18 \% (F\&E-Ausgaben von 12,69 Mrd. €). Nach Schweden verzeichnet Österreich heute die zweihöchsten F\&E-Ausgaben im europäischen Vergleich. Diese Position hat Österreich seit 2015 inne.

Insgesamt ist Österreichs Volkswirtschaft über die Jahre wissens- und forschungsintensiver geworden. Die Sachgütererzeugung, deren Dominanz vor allem in den mitteltechnologischen Branchen liegt, ist zunehmend forschungsintensiver geworden. Zudem ist der Anteil an hochtechnologischen, wissensintensiven Dienstleistungen in den vergangenen Jahren gestiegen. Es sind immer mehr Personen in F\&E beschäftigt. Die Hochschulen mit ihrem Expansionspfad - nicht nur bezüglich der Studierenden- und Abschlusszahlen, sondern auch der Verankerung des Wissensund Technologietransfers - haben wesentlich zur Entwicklung des Innovationslandes Österreich beigetragen. Ein besonderer Fokus der FTI-Politik lag seit den 90er-Jahren auf dem Auf- und Ausbau von Wissenschaft-Wirtschafts-Kooperationen, so dass auch der außeruniversitäre Sektor eine zunehmend bedeutsame Rolle einnehmen konnte.

Der Ausbruch der COVID-19-Pandemie im März 2020 hat erhebliche negative Effekte auf F\&E. Aufgrund der wirtschaftlichen Folgen der Pandemie ist absehbar, dass der Unternehmenssektor - der für knapp die Hälfte $(47,6 \%)$ aller F\&E-Ausgaben in Österreich verantwortlich ist - seine F\&EAktivitäten stark zurückfahren wird. Da die F\&E-Ausgaben der Unternehmen typischerweise prozyklisch verlaufen, besteht die Gefahr, dass Innovationen als Treiber von Wachstum und Wohlstand teilweise ausfallen. Umso entscheidender ist eine nachhaltige Entwicklung der öffentlichen F\&E-Ausgaben, um einen Rückfall der Innovationsperformance zu verhindern und ein rasches Aufholen nach der Krise zu ermöglichen.

Die Politik widmet dem Thema „F\&E“ stets eine hohe Aufmerksamkeit. So war und ist das Thema nicht nur Bestandteil sämtlicher Regierungsübereinkommen. Im Jahr 2011 wurde die „FTI-Strategie 2020" entworfen und das Ziel vorgegeben, bis 2020 die F\&E-Ausgaben auf 3,76\% des BIPs zu steigern. Österreich konnte dieses ambitionierte Ziel bislang nicht erreichen. Allerdings liegt die Forschungsquote bereits seit 2014 über dem von der EU bis 2020 angestrebten 3 \%-Ziel.

Die neue nationale FTI-Strategie und die neuen Vorgaben des Europäischen Forschungsraums setzen ambitionierte Ausgabenziele. Aber nicht nur das Ausgabenvolumen, sondern auch die Wirksamkeit der eingesetzten Mittel bestimmt, wie sehr Innovation das Wachstum und den Wohlstand steigern kann. Das Ziel der vorliegenden Studie ist es daher, die Wirkungen von Forschungsausgaben in Österreich systematisch zu analysieren und eine Quantifizierung vorzunehmen. Die Analyse soll die Wirksamkeit des Innovationssystems auf der Mikro- und Makroebene untersuchen, d.h. die Auswirkungen von Innovation auf die Leistungsfähigkeit der Unternehmen und auf das gesamtwirtschaftliche Wachstum. Folgende Fragen stehen im Mittelpunkt der Studie: 


\section{Makroebene:}

- Welche gesamtwirtschaftlichen Wirkungen auf BIP, Wertschöpfung, Produktivität, Beschäftigung und andere makroökonomische Kennzahlen gehen von Forschung und Entwicklung aus?

- Inwieweit unterscheiden sich die kurz- und langfristen Effekte?

\section{Mikroebene:}

- Wie wirkt Forschung und Entwicklung auf die Unternehmen?

- Entwickeln sich forschungsintensive Unternehmen im Hinblick auf Wachstum, Beschäftigung, Export und Überlebenschancen besser als andere, weniger F\&E intensive Unternehmen?

Darüber hinaus werden im Speziellen noch die Wirkungen der Forschungsförderung analysiert. Zu den Fragestellungen, die hier adressiert werden, zählen:

- Wie wirkt die Forschungsförderung auf private Innovation? Wie sehr kann sie zusätzliche F\&EAusgaben anregen?

- Wieviel zusätzliche Wertschöpfung und Arbeitsplätze schafft die Forschungsförderung?

- Welche gesamtwirtschaftlichen Folgen einschließlich budgetärer Kosten ergeben sich?

Der Bericht umfasst sechs Kapitel. Kapitel 2 erörtert zunächst den aktuellen Stand der theoretischen und empirischen Forschung zu den Effekten von F\&E auf Wachstum und Wohlstand und präsentiert zentrale quantitative Ergebnisse internationaler Studien. Kapitel 3 nimmt eine Bestandsaufnahme der aktuellen wirtschaftspolitischen Rahmenbedingungen auf nationaler und europäischer Ebene vor. Kapitel 4 präsentiert die Ergebnisse zu den makroökonomischen Wirkungen von Forschungsausgaben anhand eines detaillierten Wachstumsmodells der österreichischen Wirtschaft, dem WPZ Innovationsmodell. Konkret quantifiziert die Studie die Wirkungen von öffentlichen Forschungsausgaben und den Wachstumsbeitrag der Innovation, also wieviel des Wachstums ursächlich auf Innovation zurückzuführen ist. Kapitel 5 untersucht mit ökonometrischen Methoden, wie stark F\&E die Unternehmensentwicklung treibt. Die Analyse nutzt Daten der FFG zusammen mit Informationen aus der WPZ Unternehmensdatenbank. Kapitel 6 fasst die wichtigsten Ergebnisse kurz zusammen und zieht Schlussfolgerungen. 


\section{F\&E als Treiber von Wachstum und Wohlstand}

Kapitel 2 dient der Fundierung der Analysen zu Wirkungen von F\&E. Dabei wird zum einen auf zentrale Theorien und Ansätze in der wissenschaftlichen Forschung eingegangen, zum anderen werden Ergebnisse aktueller empirischer Studien zu Innovation und Wachstum aufgezeigt.

\subsection{Theoretische Fundierung}

Die Auswirkungen von F\&E stehen seit langem im Zentrum der ökonomischen Forschung. Kaum eine These ist so wenig umstritten wie jene, wonach das langfristige Wirtschaftswachstum vom technologischen Fortschritt abhängt. Bereits in den 1950er Jahren beschrieb der österreichische Ökonom Joseph Schumpeter die Innovation als Prozess „kreativer Zerstörung“, der wirtschaftlichen Strukturwandel auslöst, zur ständigen Erneuerung der Wirtschaft beiträgt und das Wachstum speist. Ausgangspunkt und Impuls dieses Wandels ist die Entstehung neuer Märkte, Produkte, Dienstleistungen, Prozesse oder Organisationsformen. Innovationen sind die Quelle für Wettbewerbsfähigkeit, Wirtschaftswachstum und Beschäftigung (Schumpeter, 1950).

Die ökonomische Forschung hat diese zentrale Rolle von Innovation sowohl in theoretischen Modellen als auch in empirischen Studien aufgegriffen. Die moderne Wachstumstheorie hat ihren Ursprung im Modell von Robert Solow (1956), der neben den Produktionsfaktoren Arbeit und Kapital den technologischen Fortschritt berücksichtigt, und zeigt, dass langfristiges Wachstum nicht allein durch Kapitalakkumulation entstehen kann. In seinem Modell und in der darauffolgenden empirischen Untersuchung wird deutlich, dass technologischer Fortschritt für jenen Teil des Wirtschaftswachstums verantwortlich ist, der nicht durch die Ausstattung einer Ökonomie mit Sachkapital erklärt werden kann (Solow, 1957). Dieses „Solow-Residuum“ wird auf technologischen Fortschritt zurückgeführt und ist damit alles andere als eine „Restgröße“.

Der technologische Fortschritt, der im Solow-Residuum sichtbar wird, ist keine exogene Größe, sondern das Resultat von Forschungsanstrengungen und Investitionsentscheidungen. In den 1980erJahren entstand daher eine umfangreiche Forschung, die das Entstehen von Wachstum endogen in Abhängigkeit von Anreizen und Rahmenbedingungen erklärt („endogene Wachstumstheorie“). Die einflussreiche Arbeit von Paul Romer (1986) berücksichtigt die Wissens-Spill-Overs im Prozess der Wissensakkumulation. Der allgemein verfügbare Wissensbestand entsteht nicht nur aus öffentlicher Grundlagenforschung, sondern auch aus den Erkenntnissen der privaten F\&E-Anstrengungen. Er hat den Charakter eines öffentlichen Guts, das für alle Unternehmen nutzbar ist. Die Unternehmen investieren in F\&E, um ihren eigenen Wettbewerbsvorteil auszubauen. Aber ein Teil des neu geschaffenen Wissens "schwappt“ auf andere Unternehmen über (daher "Spill-Overs“) und steigert deren Erfolg in ihrer eigenen F\&E. Die Entwicklung in einem Unternehmen baut auf den Erkenntnissen der anderen auf. Dadurch summieren sich die privaten F\&E-Investitionen in der Gesamtwirtschaft zu mehr als der Summe ihrer Einzelteile auf. Aus demselben Grund ist die soziale Ertragsrate der Innovation wesentlich höher als die private Rendite. Nachdem die soziale Rendite der F\&E die marktübliche Rendite deutlich übersteigt, kann die Gesamtwirtschaft stark davon profitieren, mehr private F\&E anzuregen. Das ist die zentrale Begründung für die öffentliche Forschungsförderung. Die Ressourcen sollen schließlich dorthin fließen, wo sie den höchsten Ertrag für alle zusammen erzielen. Romers (1986) Leistung liegt u.a. darin, dass er aufgezeigt hat, wie die sogenannten Wissens-Spill-Overs die Wirkungen privater F\&E-Investitionen verstärken und das Wachstum stützen.

Die Schlussfolgerung lautet: Technologischer Fortschritt ist die wichtigste Determinante des Wirtschaftswachstums. Er entsteht aus den privaten F\&E-Investitionen der Unternehmen, die in Form von Wissens-Spill-Overs positive externe Effekte auf andere Unternehmen haben, die im privaten Investitionskalkül nicht berücksichtigt sind. Diese zusätzliche soziale Rendite rechtfertigt die öffentliche Forschungsförderung. 
Mit Innovationen steigern die Unternehmen ihre Wettbewerbsfähigkeit und bauen ihre Marktanteile aus, zu Lasten der weniger erfolgreichen Konkurrenten. In einem Prozess „kreativer Zerstörung“ im Sinne Schumpeters verdrängen innovative Unternehmen die weniger leistungsfähigen. Dieser wachstumssteigernde Strukturwandel ist der zentrale Mechanismus in der „Schumpeterianischen Wachstumstheorie", die wesentlich von Aghion und Howitt (1992, 1998, 2009), Grossmann und Helpman (1991a, 1991b), und Acemoglu (2009) geprägt wurde. Demnach geht der technologische Fortschritt mit dem Verdrängen etablierter Anbieter durch neue Wettbewerber einher, die mit erfolgreicher Innovation bessere Produkte und Dienstleistungen anbieten. Daraus entsteht ein großer Anreiz für neue Anbieter, F\&E zu betreiben, um mit besseren Produkten und Diensten Marktanteile zu gewinnen und weniger leistungsfähige Anbieter zu verdrängen. Der Wettbewerb um Marktanteile zwingt auch die großen Konzerne, in F\&E investieren, um ihre Produktpalette laufend zu erneuern und erfolgreich zu bleiben.

Das Innovationssystem eines Landes besteht aus mehreren zusammenhängenden Pfeilern, wie Abbildung 1 schematisch darstellt: Im Zentrum stehen die Akteure, die unmittelbar und mittelbar an der Schaffung, Verbreitung und Anwendung von neuem Wissen beteiligt sind. Das sind neben den Unternehmen die Hochschulen und andere Forschungseinrichtungen, die unmittelbar durch Forschung und Entwicklung neue Produkte und Prozesse entwickeln. Die Grundlagenforschung und tertiäre Ausbildung erbringen zwei zentrale Vorleistungen für die Unternehmen, nämlich neues, patentierbares Wissen als Input in die kommerzielle F\&E, und die Ausbildung von wissenschaftlichem Nachwuchs und Forschungspersonal, welches etablierte Unternehmen und innovative Unternehmensgründungen für ihre F\&E benötigen. Die private F\&E treibt das Wachstum der Unternehmen. Daneben spielen auch das Finanzsystem mit Banken und Risikokapitalgebern eine zentrale Rolle, denn ohne Finanzierung gibt es kein Wachstum der Unternehmen. Das Risiko ist allerdings groß, sodass es vordringlich Risikokapital, d.h. risikotragendes Eigenkapital, zur Finanzierung von jungen, innovativen Unternehmen braucht. Erst im Verhältnis dazu sind zusätzliche Kredite zur Investitionsfinanzierung möglich.

Die Unternehmen reagieren mit ihren Innovationen auf die sich verändernden Bedürfnisse und Werte der Konsumentinnen und Konsumenten. Umgekehrt können ganz neue Designs und Angebote neue Bedürfnisse bei den Nachfragerinnen und Nachfragern wecken, wie die aktuelle Digitalisierung der Wirtschaft gerade anschaulich illustriert. Ebenso profitiert die Grundlagenforschung von einem attraktiven Standort, der maßgeblich von der Infrastruktur, dem Bildungssystem und den wirtschaftlichen Rahmenbedingungen beeinflusst wird, denn es herrscht weltweiter Wettbewerb um Spitzenforscherinnen und -forscher, wissenschaftliche Nachwuchstalente und die besten Studierenden (Keuschnigg et al., 2017).

Entscheidend für die Wirkung des Innovationssystems ist allerdings nicht nur das Volumen der F\&EAusgaben, sondern auch die Qualität des Mitteleinsatzes. Zudem kommt es auf das Zusammenspiel und die Vernetzung der einzelnen Akteure sowohl auf regionaler, nationaler als auch auf globaler Ebene an (Voßkamp und Schmidt-Ehmcke, 2006). Insbesondere die Interaktionen innerhalb des Innovationssystems führen zu wechselseitigen Lerneffekten, was die Produktivität der Akteure und die Qualität der Innovationen erhöht (Akcigit et al., 2018). Als Beispiel für die Komplementarität zwischen den einzelnen Aktivitäten mag das Zusammenwirken von öffentlicher Grundlagenforschung und angewandter privater F\&E dienen. Die öffentliche Grundlagenforschung wird nicht ihr volles Potential für die Gesellschaft entfalten, wenn es an der gelungenen Kommerzialisierung in der Privatwirtschaft mangelt. Die private F\&E wird unter ihrem Potential bleiben, wenn es bereits an den Vorleistungen der öffentlichen Forschungsinstitutionen mangelt, also an neuen patentierbaren Ideen und verfügbarem Forschungspersonal mit einer Ausbildung auf dem letzten Stand der Forschung. 


\section{Abbildung 1: Akteure und Interaktionen im Innovationssystem}

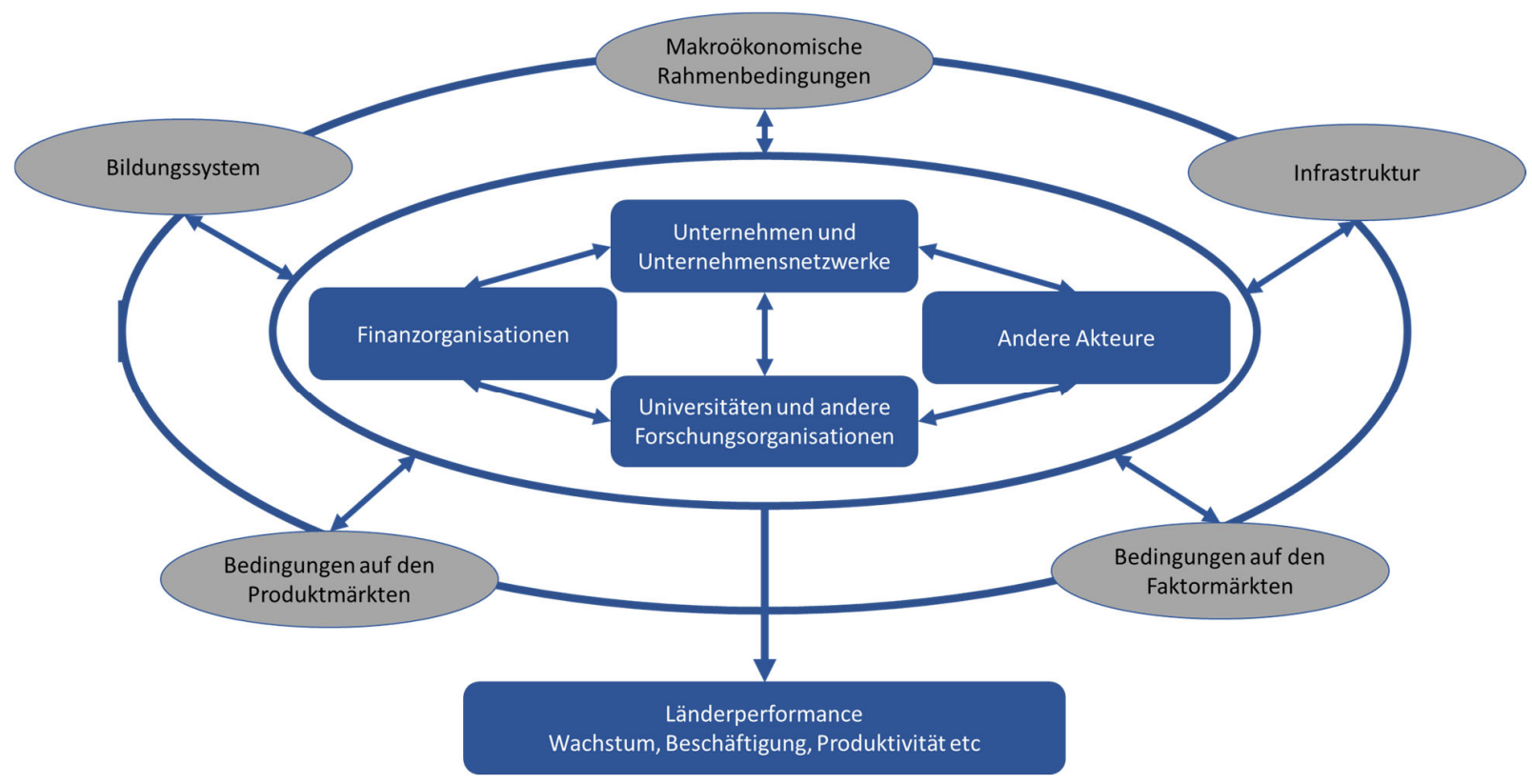

Darstellung: WPZ Research in Anlehnung an OECD (1999).

Die Wirkungen des Innovationssystems beginnen auf der Mikroebene der Unternehmen und enden im makroökonomischen Wachstum. Auf der Mikroebene sind die Fähigkeiten der Unternehmen zu erfolgreicher F\&E und die Wirkungen auf die Unternehmensentwicklung zu untersuchen. Dabei stellt sich auch die Frage, wie stark der Wissenstransfer zwischen Hochschulen und Unternehmen zur Leistungsfähigkeit des Innovationssystems beitragen kann (OECD, 1999). Für die Produktivität eines Unternehmens spielt F\&E eine zweifache Rolle. Erstens kann F\&E die Kosten senken (Prozessinnovationen) oder zu völlig neuen oder wesentlich verbesserten Produkten und Dienstleistungen führen (Produktinnovationen). Beides steigert die Nachfrage. Zweitens wachsen innovative Unternehmen schneller und verdrängen mit ihren neuen Produkten ineffiziente Mitbewerber („kreative Zerstörung“). F\&E ist nicht nur für den Erfolg eines einzelnen Unternehmens ausschlaggebend, sondern steigert damit auch die Produktivität der Gesamtwirtschaft. Mit Innovation erzielt derselbe Ressourceneinsatz einen größeren Output und steigert letztlich das ProKopf-Einkommen (Hall, 2011).

Die Aussicht auf höhere Marktanteile und höhere Gewinne motiviert die Unternehmen zu F\&EInvestitionen, die neues Wissen und Know-how schaffen. Andere Akteure, wie Forscherinnen und Forscher, Unternehmen oder andere Länder, können wenigstens teilweise vom neuen Know-how und Wissen profitieren und so ihre technologische Leistungsfähigkeit steigern. Der Nutzen von neuem Wissen lässt sich eben nicht (vollständig) beschränken. Die Verbreitung des Wissens (Spill-Overs) führt dazu, dass Innovationen nicht nur für die eigentlichen Innovatoren Erträge abwerfen, sondern auch andere Akteure davon profitieren. Dadurch entstehen zusätzliche „externe Erträge“. Da die SpillOvers oft nicht rivalisierend und nicht ausschließbar sind (d.h. ihre Nutzung ist nicht verhinderbar), können die investierenden Unternehmen schwerlich einen Preis dafür verlangen und zusätzliche Erlöse erwirtschaften. Genau aus diesem Grund tendieren private Unternehmen dazu, zu wenig in Innovation zu investieren, als gesellschaftlich optimal wäre. Es liegt daher Marktversagen vor. Weil diese externen Zusatzerträge der Innovation in der privaten Rendite nicht aufscheinen, aber für die gesamte Gesellschaft sehr relevant sind, liegt die soziale Innovationsrendite deutlich höher als die private Rendite. Die öffentliche Hand sollte daher aktiv werden, um die Innovationstätigkeit auf ein 
höheres Niveau anzuheben (Arrow, 1962). Schließlich sollen die Ressourcen dorthin fließen, wo für das ganze Land der Ertrag am höchsten ist.

Aktuelle Forschungsergebnisse von Akcigit et al. (2013) zeigen, dass die Spill-Overs in der Grundlagenforschung wesentlich höher sind als in der angewandten Forschung, weshalb eine gezielte Förderung von Grundlagenforschung sinnvoll ist, um ein gesamtgesellschaftlich optimales Niveau an F\&E-Innovationen zu erreichen. Dadurch ließen sich Fehlallokationen des Faktors Arbeit Überinvestition in angewandte Forschung, Unterinvestition in Grundlagenforschung - reduzieren und ein höheres Wirtschaftswachstum sowie eine höhere Wohlfahrt erreichen.

Neben diesen Wissens-Spill-Overs können mangelnde Informationen dazu führen, dass die Geldgeber die Chancen (und Risiken) innovativer Vorhaben nicht erkennen oder nicht richtig beurteilen. Daher können gerade sehr innovative Projekte oft keine Finanzierung erhalten, obwohl sie ein hohes Potential haben und für die Gesellschaft sehr rentabel wären (Hall, 2002). Informationsprobleme sind ebenfalls ein Marktversagen (Markman et al., 2001). Das Problem ist gerade bei jungen, innovativen Wachstumsunternehmen sehr verbreitet, wo ein hoher Investitions- und Finanzierungsbedarf auf eine geringe Selbstfinanzierungskraft trifft. Auch in diesem Fall sind staatliche Initiativen sinnvoll, wie z.B. Programme zur Belebung der Risikokapitalfinanzierung. Auch die Forschungsförderung führt den Unternehmen dringend benötigte Finanzmittel zu und kann zur Entschärfung des Finanzierungsproblems beitragen. Howell (2017) zeigt diesbezüglich anhand des US-amerikanischen Small Business Innovation Research Programms, dass durch die öffentliche F\&E-Förderung die Wahrscheinlichkeit, Wagniskapital zu erhalten, sprunghaft von $10 \%$ auf $20 \%$ ansteigt.

Die öffentliche Hand darf sich allerdings nicht ausschließlich auf den Ausgleich von Marktversagen und das Setzen von Rahmenbedingungen beschränken, sondern soll eigenständige Impulse durch Investitionen in F\&E setzen und selbst neue Märkte formen. Das gilt insbesondere für Bereiche, in denen gesellschaftliche Herausforderungen wie die Klimakrise, die Digitalisierung oder die öffentliche Gesundheit zu lösen sind und privates Kapital aufgrund des hohen Risikos oder mangels ausreichender Rendite ausbleibt (Mazzucato, 2013). Wenn F\&E zur Lösung der großen gesellschaftlichen Herausforderungen beiträgt, verbessert sie auch direkt den gesellschaftlichen Wohlstand.

Am Beispiel der Pharmaindustrie zeigt sich, dass F\&E-Aktivitäten einen langen Atem brauchen. Die Entwicklung von neuen Wirkstoffen und Medikamenten ist zeitaufwendig und kostspielig. Zwischen der F\&E-Investition, der Entdeckung und der endgültigen Zulassung des Wirkstoffs liegt üblicherweise viel Zeit. Toole (2012) schätzt anhand von US-amerikanischen Patentdaten aus den Jahren 1955 bis 1996, dass die Zeitverzögerung einer erfolgreichen Investition in die Grundlagenforschung bei ca. 17-24 Jahren liegt. Im Vereinigten Königreich sind es im Bereich der Herz-Kreislauf-Erkrankung etwa 10-25 von der Forschung bis zur Behandlung (Health Economics Research Group et al., 2008).

\subsection{Empirische Ergebnisse rezenter Studien}

Die in der Theorie herausgearbeiteten Zusammenhänge zwischen Innovation, technologischem Wandel und Wachstum sind mit einer Vielzahl von empirischen Studien belegt. Die Auswirkungen von F\&E auf Produktivität und Wirtschaftswachstum sind allerdings nicht punktgenau geschätzt, sondern unterscheiden sich zum Teil je nach Methode, untersuchten Ländern und Brachen oder nach dem Analysezeitraum.

Ein Vergleich 19 europäischer Länder im Beobachtungszeitraum 1989-2014 berücksichtigt sechs Indikatoren der Innovation, misst ihren Einfluss auf das Wirtschaftswachstum und bestätigt den positiven Zusammenhang (Maradana et al., 2017). Für Österreich wird ein positiver Einfluss von Patentanmeldungen auf das Wirtschaftswachstum identifiziert. Der Effekt ist teilweise wechselseitig, indem Wirtschaftswachstum umgekehrt auch das Ausmaß an Patentanmeldungen erhöht. Wachstum 
steigert den Marktwert neuer Erfindungen und macht damit F\&E-Investitionen profitabler. Hinsichtlich der F\&E-Ausgaben und der Anzahl wissenschaftlicher Fachartikel je 1.000 Einwohnerinnen bzw. Einwohner identifizieren die Autorinnen und Autoren für Österreich einen positiven Effekt des BIP-Wachstums auf die F\&E-Quote und die Publikationsrate. Auch stellen sie einen positiven Effekt des Bevölkerungsanteils der ForscherInnen auf das BIP-Wachstum fest.

Eine aktuelle Studie von Anzoategui et al. (2019) für die USA zeigt, dass die langsame wirtschaftliche Erholung und die schwachen Produktivitätsgewinne nach der Finanzkrise 2008 eng mit F\&E verbunden sind. Bloom et al. (2020) zeigen ebenfalls, dass die Forschungsproduktivität in den USA in den letzten Jahren deutlich zurückgegangen ist. Sowohl die Schaffung neuer Technologien durch F\&E als auch die Diffusion und Adoption dieser Innovationen erfolgen in der Regel prozyklisch. In einer Krise sinkt die Nachfrage nach neuen innovativen Produkten und Diensten und schwächt damit die Innovationsleistung der Wirtschaft. Schon vor der Krise ging die Intensität in der Nutzung von Innovationen zurück und verlangsamte das Produktivitätswachstum. Die Schwächung der F\&EEffizienz verhinderte einen raschen Produktivitätsanstieg nach der Krise. Der Wirtschaftseinbruch im Zuge der COVID-Krise dürfte das Ausmaß der Finanzkrise übertreffen. Man kann vermuten, dass ein ähnliches Muster der F\&E-Entwicklung zu erwarten ist und sich die Erholung nach der COVIDKrise verlangsamt.

\section{Abbildung 2: Beitrag von F\&E zum Wirtschaftswachstum in Europa, 2010-2016}

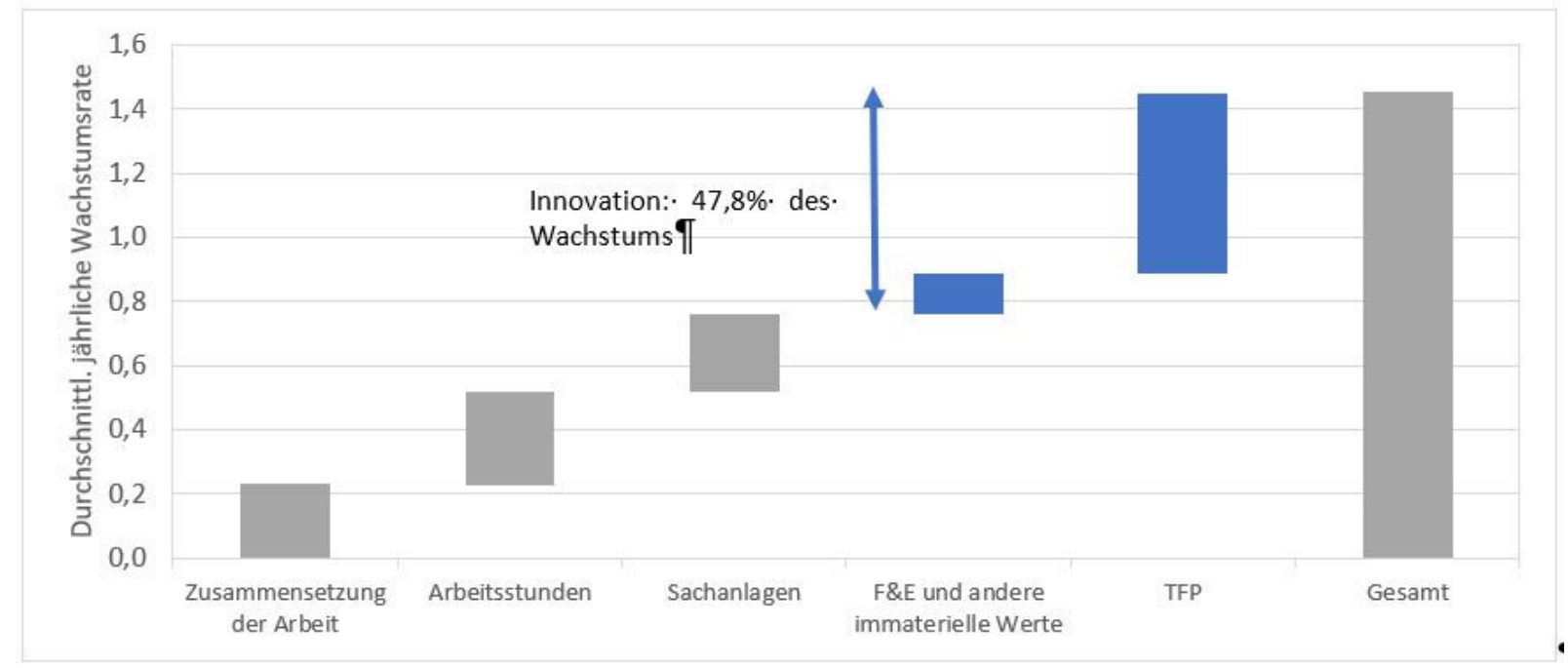

Quelle: Europäische Kommission (2020c), Darstellung: WPZ Research, Anm.: Daten von 19 EU-Mitgliedstaaten BE, CZ, DE, DK, EE, ES, FR, IT, LV, LT, LU, HU, NL, AT, RO, SI, SK, FI und SE.

Auch aktuelle Ergebnisse der EU-Kommission (2020c) zeigen, dass F\&E und immaterielle Investitionen einen erheblichen Anteil der Produktivitätsentwicklung erklären können. Mittels Wachstumszerlegung ("growth accounting") wird darin der Beitrag von Kapital, F\&E und anderen materiellen und immateriellen Komponenten zum Wachstum berechnet. Die totale Faktorproduktivität (TFP) stellt dabei einen Näherungswert für den technologischen Wandel dar. Der Beitrag von Forschung, Entwicklung und Innovation ist folglich die Summe aus immateriellen Werten wie F\&E und dem technologischen Fortschritt. Die Analyse zeigt, dass 47,8 \% und damit fast die Hälfte des Wirtschaftswachstums in Europa von 2010 bis 2016 auf Forschung, Entwicklung und Innovation zurückzuführen sind (siehe Abbildung 2). Das ist weniger als vor der Wirtschafts- und Finanzkrise (1995-2007), als noch 62 \% des Wirtschaftswachstums in Europa auf Forschung, Entwicklung und Innovation zurückzuführen waren (Bravo-Biasco et al., 2013). 
Nach derselben Methodik lässt sich nicht nur das Wirtschaftswachstum, sondern auch das Wachstum der Arbeitsproduktivität innerhalb Europas zerlegen. Die Arbeitsproduktivität entspricht der realen Wertschöpfung pro tatsächlich geleistete Arbeitsstunde. Die EU-Kommission kommt zu dem Schluss, dass knapp zwei Drittel (65,7\%) des Produktivitätswachstums 2010-2016 auf Forschung, Entwicklung und Innovation zurückzuführen sind (siehe Abbildung 3). F\&E kommt damit eine eminente Bedeutung für die Produktivität innerhalb Europas zu. Einschränkend ist allerdings festzustellen, dass die Ergebnisse je nach verwendeter F\&E-Definition stark schwanken und für verschiedene Länder sehr unterschiedlich ausfallen.

Neben dieser aktuellen Wachstumszerlegung für die EU-Kommission liegen zahlreiche weitere internationale empirische Evidenzen vor, welche die Wirkungen von F\&E auf die Gesamtwirtschaft untersuchen. So berechnen auch Guellec und van Pottelsberghe de la Potterie (2004) den Beitrag des technischen Fortschritts zum Produktivitätswachstum (totale Faktorproduktivität) in 16 OECDLändern zwischen 1980 und 1998 und unterscheiden dabei zwischen öffentlichen und privaten F\&EInvestitionen. Die ökonometrische Analyse zeigt eine Elastizität unternehmerischer F\&E-Ausgaben von 0,13. Das bedeutet, dass ein Anstieg der F\&E-Ausgaben um $10 \%$ zu einem Anstieg der totalen Faktorproduktivität von 1,3 \% führt. Die Studie zeigt außerdem, dass unternehmerische F\&E im Zeitverlauf immer wichtiger geworden ist und ihr Einfluss auf die Produktivität gestiegen ist. Die Wirkungen öffentlicher F\&E-Ausgaben sind etwas höher. Ein Anstieg der öffentlichen F\&E-Ausgaben würde nämlich zu einem Anstieg der totalen Faktorproduktivität um 1,7 \% führen.

\section{Abbildung 3: Beitrag von F\&E zum Arbeitsproduktivitätswachstum in Europa, 2010-2016}

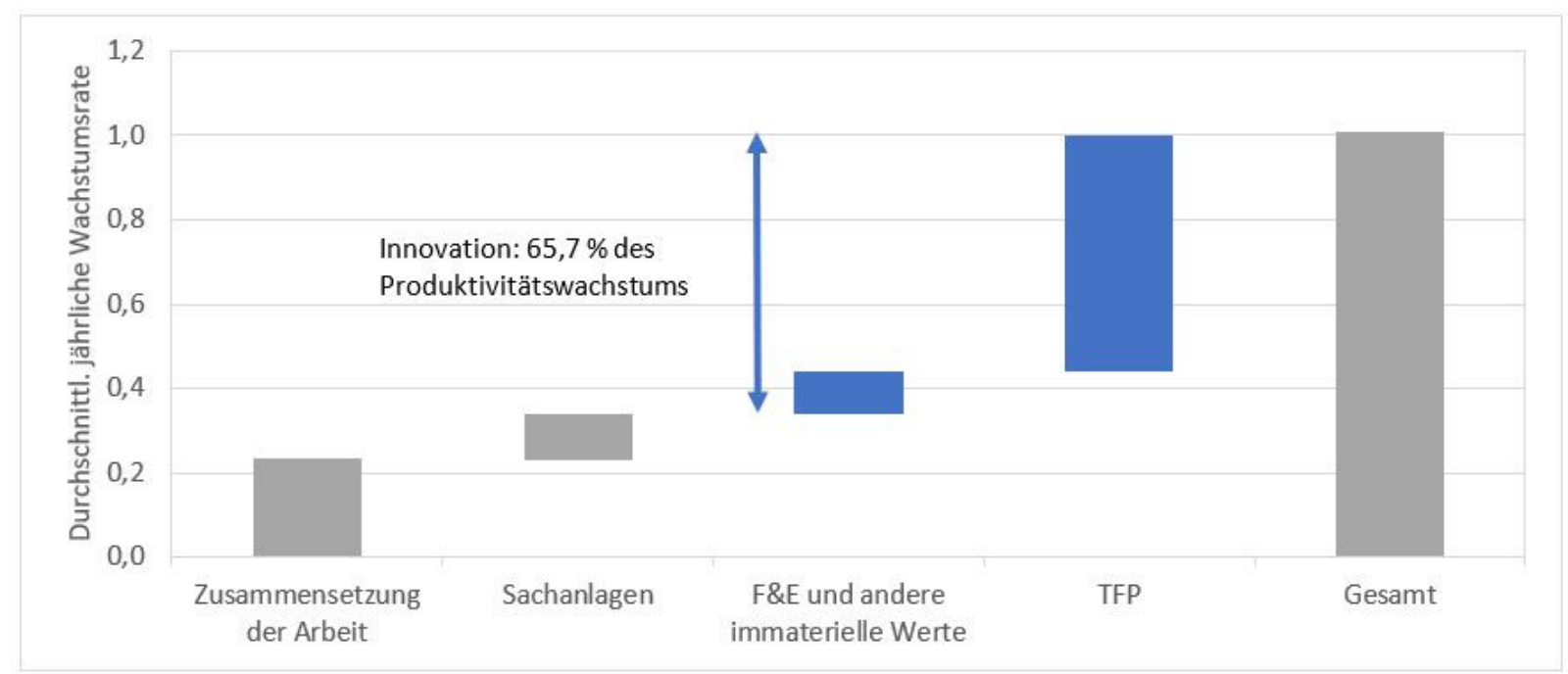

Quelle: Europäische Kommission (2020c), Darstellung: WPZ Research, Anm.: Daten von 19 EU-Mitgliedstaaten BE, CZ, DE, DK, EE, ES, FR, IT, LV, LT, LU, HU, NL, AT, RO, SI, SK, FI und SE.

Van Elk et al. (2015) untersuchen 22 OECD-Mitgliedstaaten im Zeitraum von 1963 bis 2011 und stellen etwas geringere Effekte von öffentlichen F\&E-Ausgaben auf die Produktivität fest. Die ökonometrische Analyse ergibt eine Elastizität der öffentlichen F\&E von 0,04. Steigen also die öffentlichen F\&E-Ausgaben um $10 \%$, erhöht sich die totale Faktorproduktivität um 0,4\%. Die Wirkungen unterscheiden sich zwischen den 22 Staaten allerdings stark. Für einige Länder wie die USA, Großbritannien oder Frankreich kann kein signifikanter Effekt festgestellt werde, in Norwegen $(10,8 \%)$, Finnland $(7,3 \%)$ oder Belgien $(6,4 \%)$ zeigen sich dagegen überdurchschnittlich hohe Effekte. Österreich liegt mit einer Wirkung von 0,44\% im Durchschnitt. Van Elk et al. (2015) schlussfolgern, dass die Wirkung öffentlicher F\&E-Ausgaben auf das Wirtschaftswachstum sehr unterschiedlich ausfallen kann und die nationalen Rahmenbedingungen dafür entscheidend sind. Sie 
merken auch an, dass diese Art der Elastizitäten nur den ökonomischen Effekt von öffentlicher F\&E abbildet. Ein großer Teil der öffentlichen Grundlagenforschung kann sehr wohl zur Wohlfahrt beitragen, auch wenn der Effekt auf Produktivität und Wachstum gering ist. Klinische Studien ermöglichen z.B. eine verbesserte Gesundheitsversorgung, die ein wesentlichen Bestandteil der Wohlfahrt ist, auch wenn die direkten Wachstumseffekte gering sein mögen.

Auch das Deutsche Institut für Wirtschaftsforschung (Belitz et al., 2015) berechnet anhand eines Panels von 19 OECD-Staaten von 1981 bis 2011, dass F\&E-Investitionen das Wirtschaftswachstum steigern. Wenn in den untersuchten OECD-Ländern die gesamtwirtschaftlichen Forschungsausgaben um einen Prozentpunkt wachsen, steigt kurzfristig das BIP-Wachstum um durchschnittlich 0,05 Prozentpunkte. Für Deutschland liegt der Wert mit 0,12 Prozentpunkten mehr als doppelt so hoch. Der Einfluss der F\&E im privaten und im öffentlichen Sektor ist allerdings schwer auseinanderzuhalten, weil beide Bereiche im Innovationssystem eng zusammenwirken.

Da in den letzten Jahren zahlreiche Studien erschienen sind, welche die Wirkung von F\&E auf die makroökonomische Entwicklung untersuchen, prüfen Donselaar und Koopmans (2016) in einer Metaanalyse 15 verschiedene empirische Studien, die insbesondere für OECD-Staaten durchgeführt wurden. Die Metaanalyse errechnet über alle 15 Studien hinweg eine Elastizität der totalen Faktorproduktivität hinsichtlich unternehmerischer F\&E-Ausgaben von 0,058. Eine Erhöhung der privaten F\&E-Ausgaben um $10 \%$ würde demnach zu einer um 0,58\% höheren totalen Faktorproduktivität führen. Für öffentliche F\&E-Ausgaben fällt die Elastizität laut Metaanalyse mit 0,033 etwas geringer aus. Sowohl öffentliche als auch private F\&E-Ausgaben können also ganz erheblich die Produktivität steigern und damit das Wirtschaftswachstum verstärken.

Griffith et al. (2004) zeigten empirisch, dass die Rendite von F\&E-Investitionen in Abhängigkeit von der Entfernung zur technologischen Grenze variiert und dass die Renditen für Länder, die weiter von der technologischen Grenze entfernt sind, höher ausfallen.

Für Österreich zeigt eine ältere Studie von Falk und Hake (2008) mit Daten von 1975 bis 2007, dass sowohl inländische als auch ausländische F\&E die gesamtwirtschaftliche Faktorproduktivität erheblich beeinflussen. Die forschungsintensiven Niederlassungen ausländischer Konzerne z.B. aus Deutschland sind wesentlicher Bestandteil des heimischen Innovationssystems. Der Produktivitätseffekt der gesamten F\&E-Kapazitäten in Österreich ist tendenziell höher als im Durchschnitt der OECD-Länder. Das empirische Modell zeigt, dass ein Anstieg des inländischen F\&EKapitalstocks um $10 \%$ zu einer Steigerung der gesamten Faktorproduktivität um 1,6 \% führt, im Durchschnitt der OECD-Länder sind es dagegen lediglich 1,1\%. 


\section{Wirtschaftspolitische Rahmenbedingungen}

Wie viel für F\&E ausgegeben wird, ist auch davon abhängig, wie die Rahmenbedingungen gestaltet sind, und damit einhergehend, welche Ziele und Anreize gesetzt werden. Grundsätzlich ist die FTIPolitik ein sehr agiles Politikfeld, welches sowohl auf europäischer Ebene als auch auf nationaler Ebene hohe Aufmerksamkeit auf sich zieht. In Folge werden daher die jüngsten Entwicklungen sowie die aktuellen bzw. in die Zukunft ausgerichteten FTI-Ziele auf europäischer und nationaler Ebene im Überblick dargestellt.

\subsection{Entwicklungen und FTI-Ziele auf europäischer Ebene}

Eine umfangreiche theoretische und empirische Forschung zeigt, dass F\&E und Innovation nachhaltig das Wachstum steigern (siehe Kapitel 2). Forschung und Entwicklung ist daher für die Europäische Kommission von hoher Bedeutung. Die Union hat in der Strategie „Europa 2020“ das Ziel einer europaweiten F\&E-Quote von $3 \%$ bis 2020 ausgerufen (Europäische Kommission, 2010). Im Einklang mit dieser strategischen Zielsetzung sind tatsächlich die Ausgaben für F\&E in den letzten Jahren europaweit gestiegen. Lag die F\&E-Quote der EU-27 (ohne Großbritannien) 2009 noch bei $1,97 \%$, betrug sie zehn Jahre später bereits 2,19\%. Nach den letzten Daten von 2019 hat die EU ihr Ziel allerdings nicht erreicht. Zwar konnten 20 Mitgliedstaaten ihre F\&E-Quote erhöhen, aber lediglich drei Staaten übertrafen die $3 \%$-Grenze. Das sind Schweden $(3,39 \%)$, Österreich $(3,18 \%)$ und Deutschland (3,12 \%). Österreich ist damit Spitzenreiter hinsichtlich der Ausgaben für F\&E und liegt nicht nur europaweit an zweiter Stelle, sondern auch vor führenden Innovationsnationen wie den USA $(2,82 \%)$ oder China $(2,14 \%)$.

\section{Abbildung 4: F\&E-Quote in der EU und ausgewählten Vergleichsländern, 2009 und 2019}

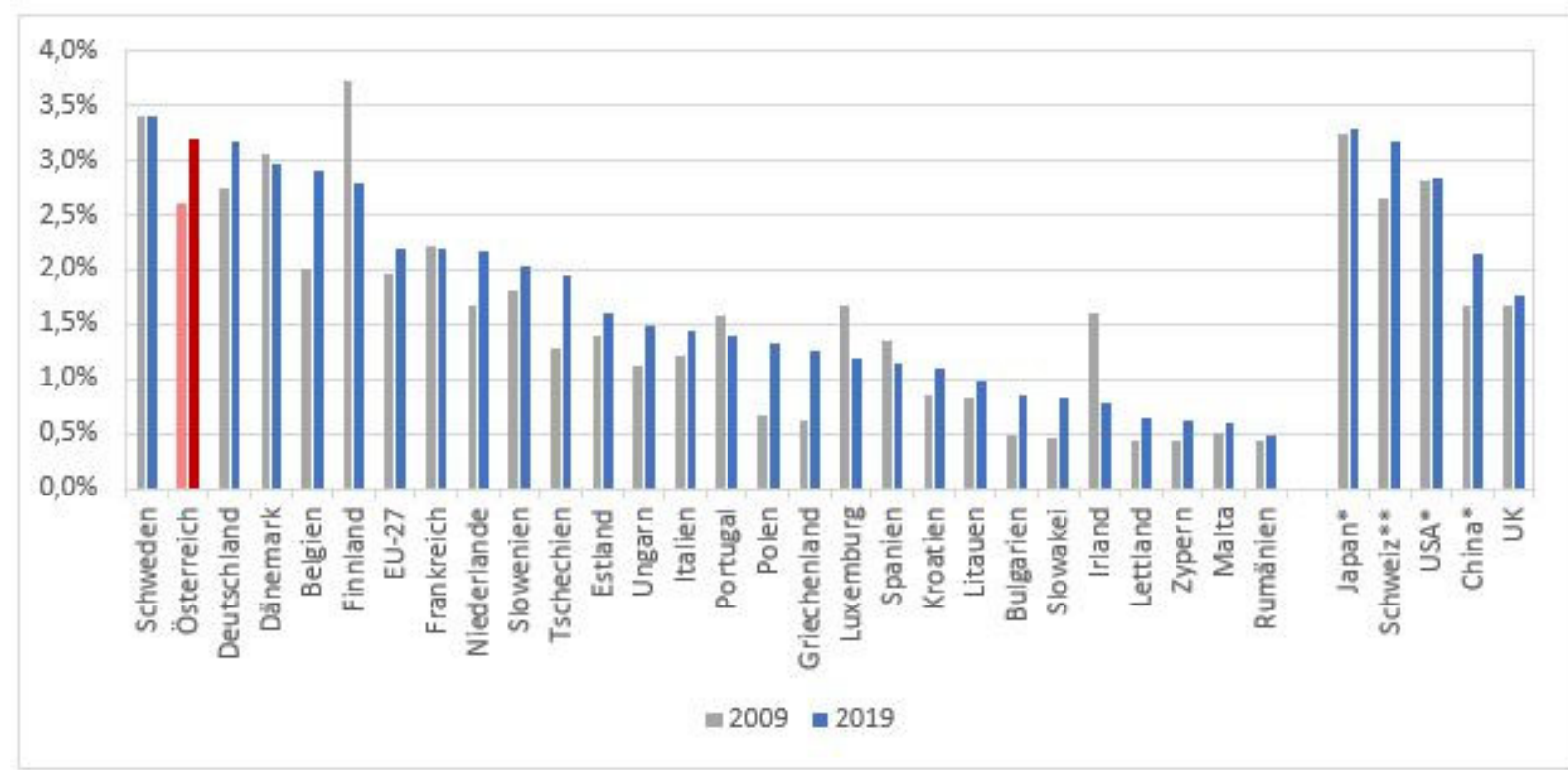

Quelle: Eurostat (2020). Anm.: * Daten von 2018; ** Daten von 2008 bzw. 2017. Eurostat weist die F\&E-Quote Österreichs 2019 mit 3,19 \% aus. Gemäß revidierter Globalschätzung der Statistik Austria liegt die F\&E-Quote bei 3,18 \%. Aufgrund der COVID-19-Pandemie war eine Globalschätzung für Österreich 2020 nicht möglich.

Nicht nur an der Entwicklung der F\&E-Ausgaben, sondern auch am F\&E-Personal zeigt sich, dass die EU und Österreich deutlich forschungsintensiver geworden sind. In Österreich lag 2008 der Anteil des F\&E-Personals (gemessen in VZÄ) an der Erwerbsbevölkerung bei 1,4 \%. Innerhalb von zehn 
Jahren stieg der Anteil bis 2018 bereits auf 1,8\% an. Österreich schlägt damit sehr deutlich den Durchschnitt der EU-27 (1,4 \%) und liegt europaweit an vierter Stelle. Dänemark (2,2 \%), Luxemburg $(1,9 \%)$ und Finnland $(1,9 \%)$ stehen an der Spitze. Sie mussten allerdings in den letzten Jahren zum Teil einen Rückgang des F\&E-Personals zu verzeichnen. Für das Jahr 2019 liegen noch keine europaweiten Daten vor.

\section{Abbildung 5: F\&E-Personal und ForscherInnen (VZÄ) an der Erwerbsbevölkerung, 2008 und 2018}

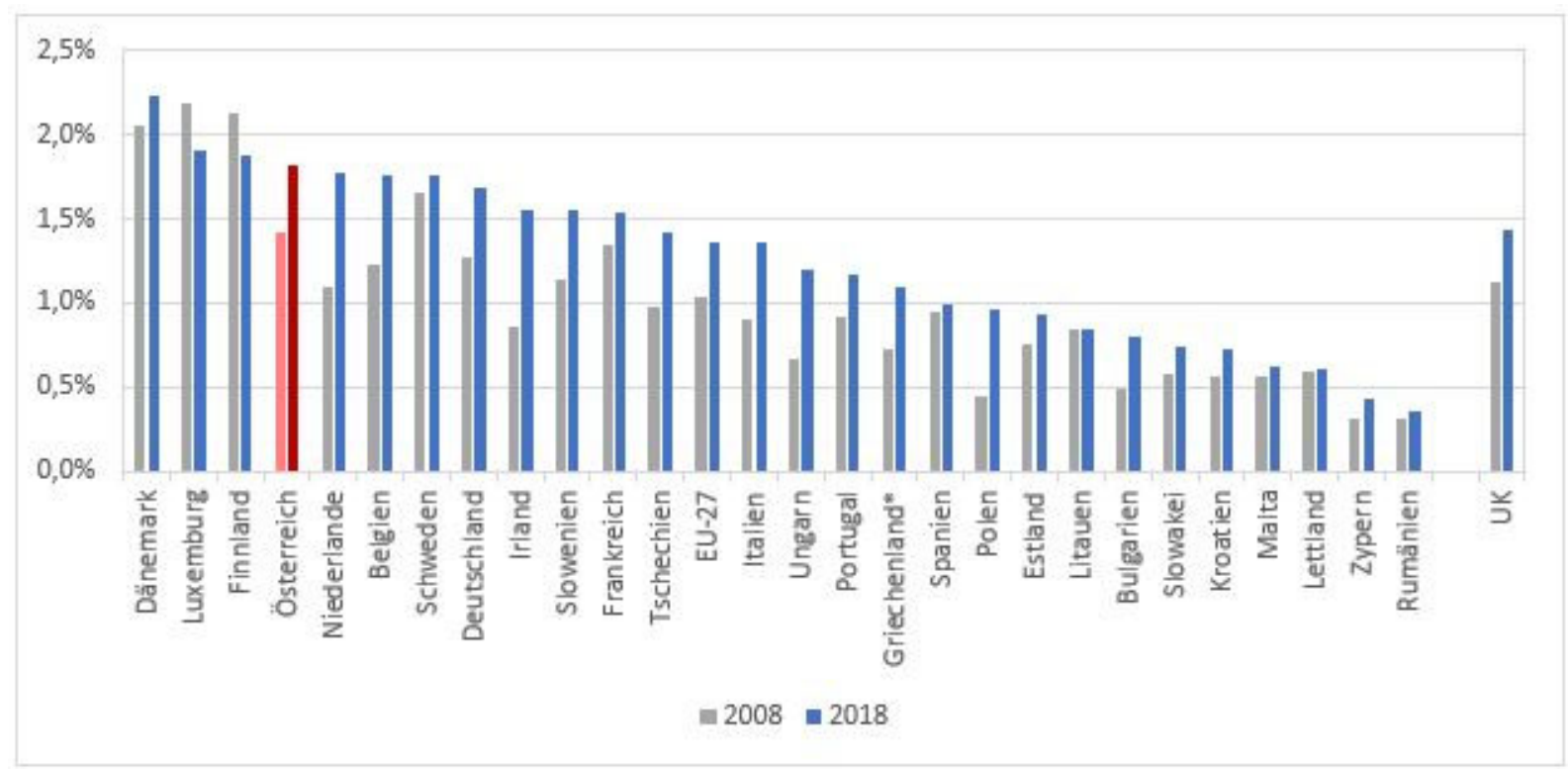

Quelle: Eurostat (2020). Anm.: * Griechenland: Wert für 2007 statt 2008.

\section{Aktuelle strategische Zielsetzungen im Europäischen Forschungsraum}

Auch nach Auslaufen der Strategie „Europa 2020“ bleibt F\&E weiterhin eine Priorität. In ihren politischen Leitlinien hat Kommissionspräsidentin Ursula von der Leyen angekündigt, „Rekordsummen in Spitzenforschung und Innovation" zu investieren und dabei die volle Flexibilität des nächsten EU-Haushalts zu nutzen, um den Schwerpunkt auf die Bereiche mit dem größten Potenzial zu legen (von der Leyen, 2019). Die Schwerpunkte werden dabei insbesondere auf der Bewältigung der Klimakrise und den Herausforderungen der Digitalisierung liegen. Disruptive Forschung und bahnbrechende Innovationen sollen den „europäischen Green Deal“ unterstützen und Europa bis 2050 klimaneutral machen (Europäische Kommission, 2020b). Ebenso sollen digitale Technologien wie Künstliche Intelligenz, das Internet der Dinge, 5G und 6G speziell unterstützt werden, damit Europa eine technologische Vorreiterstellung einnehmen kann (Europäische Kommission, 2020d).

Die COVID-19-Pandemie mag als Beispiel dienen, wie wesentlich F\&E im Gesundheitssektor ist und wie digitale Technologien neue Lösungen für Wirtschaft und Gesellschaft ermöglichen. Die Pandemie hat nicht nur zu einer gesundheitlichen, sondern auch zu einer ökonomischen und sozialen Krise geführt. Um Auswege aus der Krise zu finden, ist neben medizinischer Expertise auch das Wissen aus den anderen Disziplinen gefragt.

Der Europäische Forschungsraum (European Research Area, ERA) spielt eine Schlüsselrolle in der Bewältigung dieser Herausforderungen. Sein Ziel ist der Aufbau eines gemeinsamen Wissenschaftsund Technologieraumes in der EU. Ein gemeinsamer Binnenmarkt für Forschung und Innovation soll entstehen, der die Freizügigkeit von Forschenden, wissenschaftlichen Erkenntnissen und Innovationen fördert und die Wettbewerbsfähigkeit der europäischen Industrie stärkt. Die Ziele von 
ERA umfassen auch die Umstrukturierung der europäischen Forschungslandschaft, um mehr grenzüberschreitende Zusammenarbeit, den Aufbau einer kritischen Masse und eine Koordinierung sowie eine Verbesserung der nationalen Forschungspolitiken und -systeme zu erreichen. Im September 2020 wurde eine neue Vision von ERA präsentiert, um Europas grüne und digitale Transformation zu beschleunigen, die Widerstandsfähigkeit zu stärken und im globalen Wettlauf um neues Wissen einen Wettbewerbsvorsprung zu erzielen (Europäische Kommission, 2020a). Die EUKommission schlägt dabei folgende strategische Ziele vor, die gemeinsam mit den Mitgliedstaaten erreicht werden sollen:

1. „Priorisierung von Investitionen und Reformen: Ziele sind, den grünen und digitalen Übergang zu beschleunigen, die Wettbewerbsfähigkeit zu stärken und die Geschwindigkeit und Breite der Erholung zu steigern. Das Zusammenspiel zwischen nationalen und europäischen FuISystemen ist zu vereinfachen und zu erleichtern. Das Prinzip der Exzellenz, d. h. die Finanzierung der besten Forschenden mit den besten Ideen, bleibt der Eckpfeiler für alle Investitionen im EFR.

2. Verbesserung des Zugangs zu Exzellenz: Die Hinwendung zu mehr Exzellenz und stärkeren FuI-Systemen in der gesamten EU trägt dazu bei, dass sich bewährte Verfahren schneller in ganz Europa verbreiten. Die Mitgliedstaaten sollten ermutigt und unterstützt werden, die Leistung ihres FuI-Systems in Richtung Exzellenz zu steigern.

3. Umsetzung von FuI-Ergebnissen in der Wirtschaft: Die FuI-Politik sollte daraufabzielen, die Widerstandsfähigkeit und Wettbewerbsfähigkeit zu stärken. Sie soll das Umfeld für FuEInvestitionen der Unternehmen verbessern, den Einsatz neuer Technologien sowie der Übernahme und Sichtbarkeit von Forschungsergebnissen in Wirtschaft und Gesellschaft insgesamt ermutigen, und damit die Führungsposition Europas im globalen Technologiewettlauf sichern.

4. Vertiefung des EFR: Der EFR soll weitere Fortschritte bei der freien Zirkulation von Wissen im europaweiten FuI-System erzielen, insbesondere durch eine verbesserte Koordination und Verflechtung zwischen den nationalen Politiken. Der EFR wird angemessene Rahmenbedingungen und Inklusivität fördern und zur Entwicklung von Kompetenzen beitragen, die Forschende für eine exzellente Wissenschaft benötigen. Sie soll alle Akteure in ganz Europa verbinden, auch in der Aus- und Weiterbildung und auf dem Arbeitsmarkt" (Europäische Kommission, 2020a, S. 5).

Um diese Ziele zu erreichen, sind 14 konkrete Maßnahmen bis 2030 vorgeschlagen ${ }^{2}$. Einige davon betreffen die F\&E-Ausgaben: Das Ziel, $3 \%$ des BIP in F\&E zu investieren, war bereits in der Strategie „Europa 2020“ vorgegeben und soll nun bis 2030 erreicht werden. Darüber hinaus sollen die Mitgliedstaaten die öffentlichen F\&E-Ausgaben auf 1,25 \% des BIP erhöhen. EU-Mitgliedstaaten, die bisher unterdurchschnittlich in F\&E investierten, sind angehalten, ihre F\&E-Ausgaben in den nächsten fünf Jahren um $50 \%$ erhöhen. Aktuell weisen 20 Mitgliedstaaten unterdurchschnittliche F\&E-Ausgaben auf. Litauen, Bulgarien, Slowakei, Irland, Lettland, Zypern, Malta und Rumänien haben mit F\&E-Ausgaben von weniger als 1\% des BIP 2019 den größten Rückstand. Weitere inhaltliche Schwerpunkte sind die Einrichtung von Forschungsinfrastrukturen auf Spitzenniveau, Geschlechtergerechtigkeit und Diversität in F\&E, sowie Open Science und Open Access.

Abbildung 6 zeigt die Entwicklung des Anteils öffentlicher F\&E-Ausgaben am BIP von 2008 und 2018. Für 2019 liegen noch keine europaweiten Daten vor. Aktuell erreicht noch kein Mitgliedsstaat das neue Ziel von 1,25 \% des BIPs. Österreich besetzt aktuell mit 0,94 \% die Spitzenposition, dahinter folgen Deutschland (0,87 \%) und Schweden $(0,84 \%$ im Jahr 2017). Österreich liegt damit noch vor führenden Innovationsländern außerhalb der EU wie der Schweiz (0,87 \% im Jahr 2017), den USA $(0,65 \%)$ oder Japan (0,48\%). Der Durchschnitt der EU-27 (ohne Großbritannien) liegt 2018 bei

\footnotetext{
2 Vgl. https://ec.europa.eu/info/files/new-european-research-area en bzw. Europäische Kommission (2020a, S. 23).
} 
0,65 \%. Die Abbildung verdeutlicht auch, dass die Entwicklung der öffentlichen F\&E-Ausgaben in den letzten zehn Jahren sehr unterschiedlich verlaufen ist. Während einige Länder wie Deutschland, Schweden oder Dänemark ihre öffentlichen F\&E-Ausgaben gegenüber 2008 leicht steigern konnten, haben anderen Staaten wie Portugal, Spanien, Irland oder Rumänien deutlich an Terrain verloren.

\section{Abbildung 6: Anteil der öffentlichen F\&E-Ausgaben am BIP, 2008 und 2018}

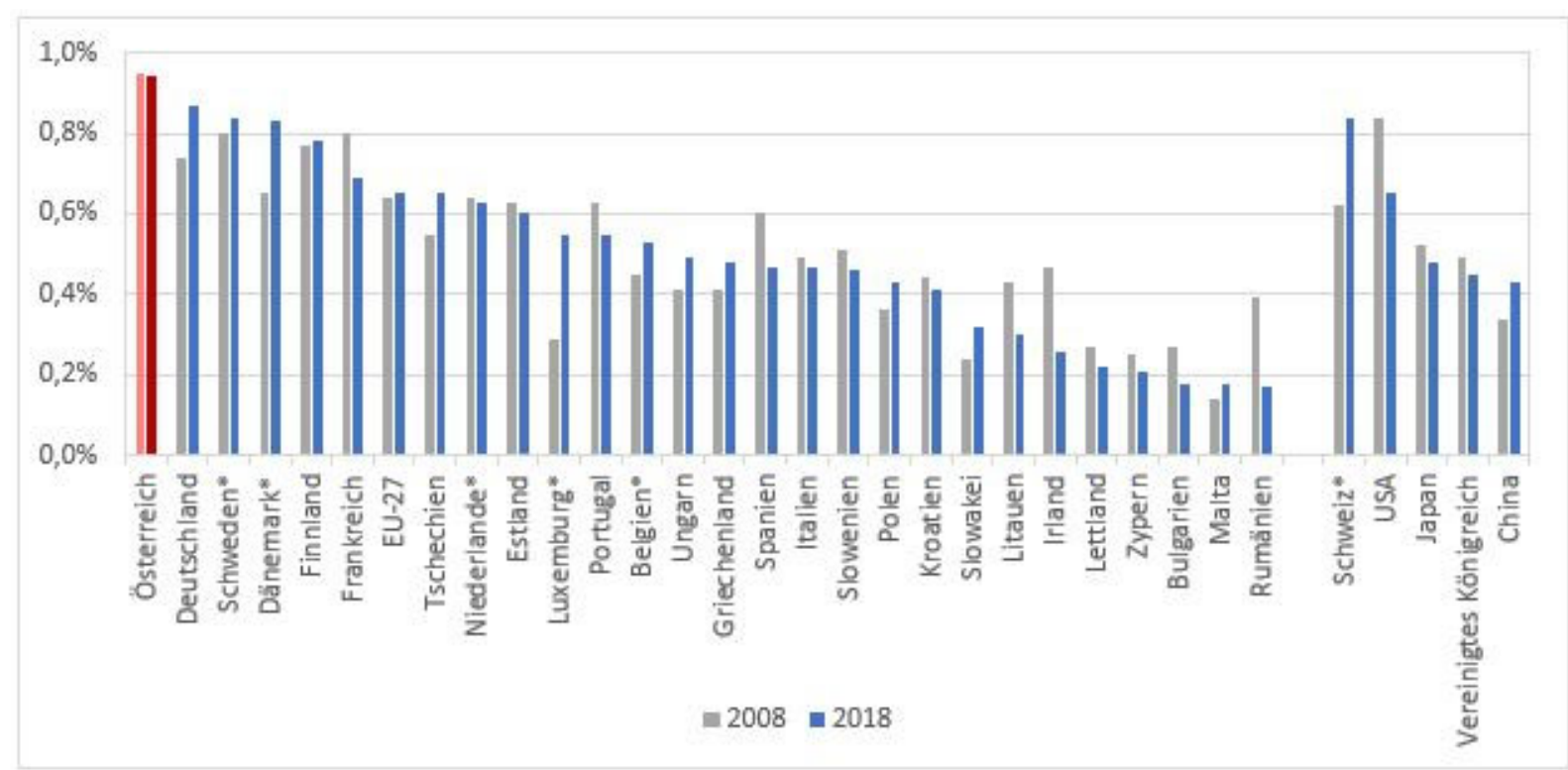

Quelle: Eurostat (2020). Anm.: * letztverfügbare Daten ausgewählt

\section{Das neue EU-Forschungsrahmenprogramm Horizon Europe}

Neben den neuen Zielsetzungen und Maßnahmen im Europäischen Forschungsraum hat auch das neue EU-Rahmenprogramm für Forschung und Innovation Horizon Europe für die Jahre 2021-2027 neue Schwerpunkte gesetzt und erstmals F\&E-Missionen formuliert. Diese lauten:

\section{Den Krebs besiegen}

Ziel bis 2030: mehr als 3 Mio. weitere Leben vor Krebs retten.

\section{Ein klimaresistentes Europa schaffen}

Ziel bis 2030: Europa auf die Bewältigung von Klimaveränderungen vorbereiten und die Transformation in ein klimaresistentes und gerechtes Europa bis 2030 beschleunigen.

3. Mission Starfish 2030

Ziel bis 2030: Ozeane und Gewässer wiederherstellen und die „blaue Wirtschaft" entkarbonisieren.

4. 100 klimaneutrale Städte bis 2030

Ziel bis 2030: systemischen Umwandlung von 100 europäischen Städten in Richtung Klimaneutralität und Transformation dieser Städte in Innovationszentren für andere Städte.

\section{Pflege des Bodens ist Pflege des Lebens}

Ziel bis 2030: 75 \% aller Böden sollen gesund für Ernährung, Mensch, Natur und Klima sein.

\footnotetext{
3 Vgl. https://ec.europa.eu/info/horizon-europe-next-research-and-innovation-framework-programme/missions-horizon-europe en, aufbauend auf Mazzucato (2018).
} 
Diese Missionen und thematischen Schwerpunkte werden die nächsten Jahre prägen. Für Horizon Europe (2021-2027) ist insgesamt ein Volumen von 84,9 Mrd. € budgetiert. ${ }^{4}$ Allerdings: Laut einer rezenten Studie kann die die missionsorientierte FTI-Politik von China für die EU kein Vorbild sein, denn dort kam es in den letzten Jahren zu einem rapiden Rückgang der Forschungsproduktivität, obwohl die F\&E-Ausgaben stark angestiegen sind (Boeing und Hünermund, 2020).

\section{Exkurs: Direkte und indirekte F\&E-Förderung in der EU}

Aufgrund positiver Spill-Over-Effekte fallen positive externe F\&E-Erträge auch bei anderen Unternehmen an. Da dieser Zusatznutzen wie bei öffentlichen Gütern nicht ausschließbar ist, können F\&E-betreibende Unternehmen für diese externen Erträge keine Erlöse erzielen. Wer nur einen Teil der Erträge verbuchen kann, aber alle Kosten tragen muss, investiert weniger in F\&E als gesellschaftlich optimal wäre. Um die Innovationsanreize zu stärken, kann die öffentliche Hand die externen Erträge mit Direktzahlungen oder Steueranreizen nach dem Verursacherprinzip ersetzen.

Steuernachlässe bzw. Steuerguthaben für F\&E-Ausgaben wirken sofort, sobald ein Unternehmen sie beantragt. Ein administrativer Aufwand entsteht dabei allenfalls in Abgrenzungsfragen, was genau als F\&E-Ausgaben gelten kann. Direkte F\&E-Förderungen werden dagegen durch spezielle Fördergesellschaften vergeben, welche die Förderungswürdigkeit beurteilen. Während bei der indirekten (steuerlichen) Förderung den Marktkräften die Auswahl der F\&E-Investitionen überlassen bleibt, erlauben direkte Förderungen eine strategische Schwerpunktsetzung. Beispiele sind die Ausrichtung auf Klimakrise, demographischen Wandel, Digitalisierung oder Bekämpfung spezieller Krankheiten oder aktuell der COVID-19-Epidemie, um das Potential des Innovationssystems für die Bewältigung dieser Herausforderungen zu nutzen. So kann der Staat öffentliche Gelder gezielt in Projekte mit hohem gesellschaftlichen Impact lenken. Das Auswahlverfahren der Fördergesellschaften kann zudem kostspielige Mitnahmeeffekte reduzieren. Für Unternehmen entsteht dadurch allerdings ein Antragsrisiko, wenn sie trotz Aufwand die Genehmigung nicht schaffen. Kleinere Unternehmen wie innovative Start-ups, die noch wenig Gewinn erzielen und daher kaum Steuerabzüge erhalten, profitieren stärker von direkten F\&E-Förderungen, wenn sie im Auswahlverfahren erfolgreich sind. Zudem sind die Fördersätze bei der direkten Forschungsförderung für kleine und mittlere Unternehmen in der Regel höher.

Im internationalen Vergleich hat die steuerliche F\&E-Förderung im letzten Jahrzehnt stark zugenommen. Die überwiegende Mehrheit der OECD- und der EU-Mitgliedstaaten hat eine steuerliche F\&E-Förderung etabliert. In Deutschland wurde ein solches Instrument 2020 eingeführt. 5 Die Schweiz hat mit der jüngsten Steuerreform STAF ab 2020 verpflichtend auf kantonaler Ebene eine Patentbox-Regelung eingeführt und damit ebenfalls einen Schritt Richtung steuerlicher F\&EFörderung getan. ${ }^{6}$ Patentboxen stehen allerdings international in der Kritik, den aggressiven Steuerwettbewerb zu befeuern und die Steuerbasis zu untergraben. Die OECD hat mit dem „Inclusive Framework on Base Erosion and Profit Shifting" 7 umfassende Vorschläge erarbeitet, um die Verminderung steuerlicher Bemessungsgrundlagen und das grenzüberschreitende Verschieben von Gewinnen durch multinationale Konzerne einzudämmen. Die Expertenkommission für Forschung und Innovation in Deutschland (EFI) empfiehlt Patentboxregelungen international zur Gänze

4 Vgl.

https://ec.europa.eu/info/sites/info/files/about the european commission/eu budget/mff factsheet agreement en web 20.11.pdf

5 Vgl.

https://www.bundesfinanzministerium.de/Content/DE/Standardartikel/Themen/Steuern/Steuerliche Themengebiete/Forschungs

zulage/2020-04-08-forschungszulage-introartikel.html

6 Vgl. https://www.bsv.admin.ch/bsv/de/home/sozialversicherungen/ahv/reformen-revisionen/staf.html

Vgl. http://www.oecd.org/tax/beps/ 
abzuschaffen (EFI, 2016). Aktuell beschränken sich nur wenige Industrienationen wie etwa Finnland oder Luxemburg ausschließlich auf direkte Forschungsförderung.

Die Wirkung der steuerlichen Forschungsförderung ist empirisch belegt. Bloom et al. (2002) berechnen mit Paneldaten von OECD-Ländern für die Jahre 1979-1997, dass eine Senkung der F\&EKosten durch steuerliche Förderung um ein Prozent mit einer langfristigen Steigerung der F\&EAktivität um ca. ein Prozent assoziiert ist. Dechezlepretre et al. (2016) analysieren anhand einer Steuerreform in Großbritannien, bei der die F\&E-Steuerabzüge für KMU von $150 \%$ auf $175 \%$ und für große Unternehmen von $125 \%$ auf $130 \%$ angehoben wurden, welche Wirkungen indirekte F\&EFörderungen entfalten. Die Erhöhung der steuerlichen F\&E-Förderung bewirkte etwa eine Verdoppelung der F\&E-Ausgaben und wirkte insbesondere bei jenen Unternehmen anreizfördernd, die bereits zuvor hohe F\&E-Ausgaben getätigt hatten.

Abbildung 7 zeigt die Ausgaben für direkte (steuerliche) und indirekte F\&E-Förderung in Prozent des BIP. Demnach bieten Belgien $(0,36 \%)$, Frankreich $(0,35 \%)$ und Italien $(0,27 \%)$ im internationalen Vergleich die stärkste F\&E-Förderung an, vor allem durch indirekte Förderung. Österreich wendet insgesamt $0,24 \%$ des BIP $(0,08 \%$ direkt, $0,16 \%$ indirekt) für F\&E-Förderung auf und rangiert damit an vierter Stelle. Im Vergleich zu anderen Staaten ist das Verhältnis zwischen direkter und indirekter Forschungsförderung in Österreich recht ausgeglichen, wenngleich in den letzten Jahren die indirekte Förderung etwas an Bedeutung gewonnen hat

Abbildung 7: Direkte und indirekte F\&E-Förderung als Anteil des BIP, 2017

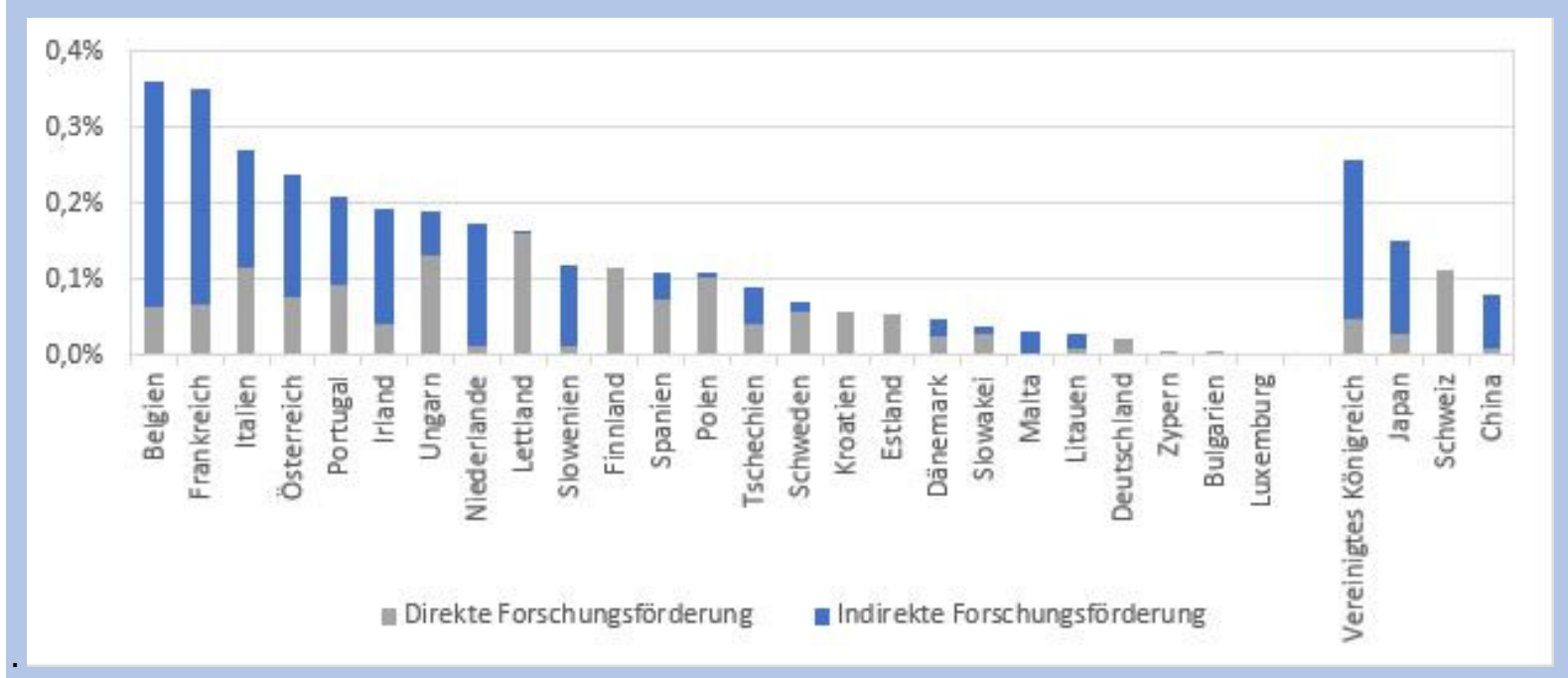

Quelle: OECD (2020).

\subsection{Entwicklungen und FTI-Ziele auf nationaler Ebene}

Auch Österreich hat sich zum Ziel gesetzt, verstärkt in F\&E zu investieren. In der 2011 verabschiedeten FTI-Strategie wurde eine F\&E-Quote von 3,76\% bis zum Jahr 2020 angestrebt. Ebenso sollte Österreich bis dahin zu den Innovation Leadern aufsteigen, also zu den führenden Innovationsnationen, wie sie im European Innovation Scoreboard definiert werden. Wie der aktuelle Review der FTI-Strategie zeigt, konnte die Republik zwar eine Vielzahl von Maßnahmen erfolgreich umsetzen, aber den Vorstoß zur Gruppe der Innovation Leader nicht schaffen (BMBWF et al., 2019). Auch eine F\&E-Quote von 3,76 \% erscheint aktuell außer Reichweite. Nach Statistik Austria liegt die F\&E-Quote 2019 bei 3,18 \%.

Wie Abbildung 8 zeigt, haben sich die F\&E-Ausgaben in den letzten zehn Jahren stetig erhöht. Die F\&E-Ausgaben summierten sich 2019 auf insgesamt 12,69 Mrd. € auf. Im Jahr 2009 waren es rund 
7,5 Mrd. €. Das entspricht einem Zuwachs von $70 \%$. Im selben Zeitraum fiel das nominale Wirtschaftswachstum mit $38 \%$ nur etwa halb so hoch aus. Die österreichische Wirtschaft ist forschungsintensiver geworden.

Der Bund investierte dabei rund 3,12 Mrd. €, was etwa ein Viertel $(24,6 \%)$ der gesamten in Österreich durchgeführten F\&E ausmacht. Die Bundesländer gaben 2019 rund 0,55 Mrd. € für F\&E aus, das entspricht einem Anteil von 4,3\%. Damit verantwortete die öffentliche Hand (Bund, Bundesländer und sonstige öffentliche Finanzierung) mit 3,83 Mrd. € fast ein Drittel (30,2\%) aller F\&E-Ausgaben. Der Anteil der öffentlichen F\&E-Ausgaben am BIP belief sich 2019 auf 0,96 \%. Im Hinblick auf den EU-Zielwert von 1,25\% des BIPs für 2030 besteht also noch erheblicher Aufholbedarf. Zudem war der BIP-Anteil in den letzten Jahren Schwankungen unterworfen und folgte keinem klaren Trend (siehe Tabelle 1). Um das EU-Ziel zu erreichen, muss sich die Republik erst noch auf einen klaren Wachstumspfad für die öffentlichen F\&E-Ausgaben verpflichten.

\section{Abbildung 8: Entwicklung F\&E-Ausgaben und F\&E-Quote in Österreich, 2009-2019}

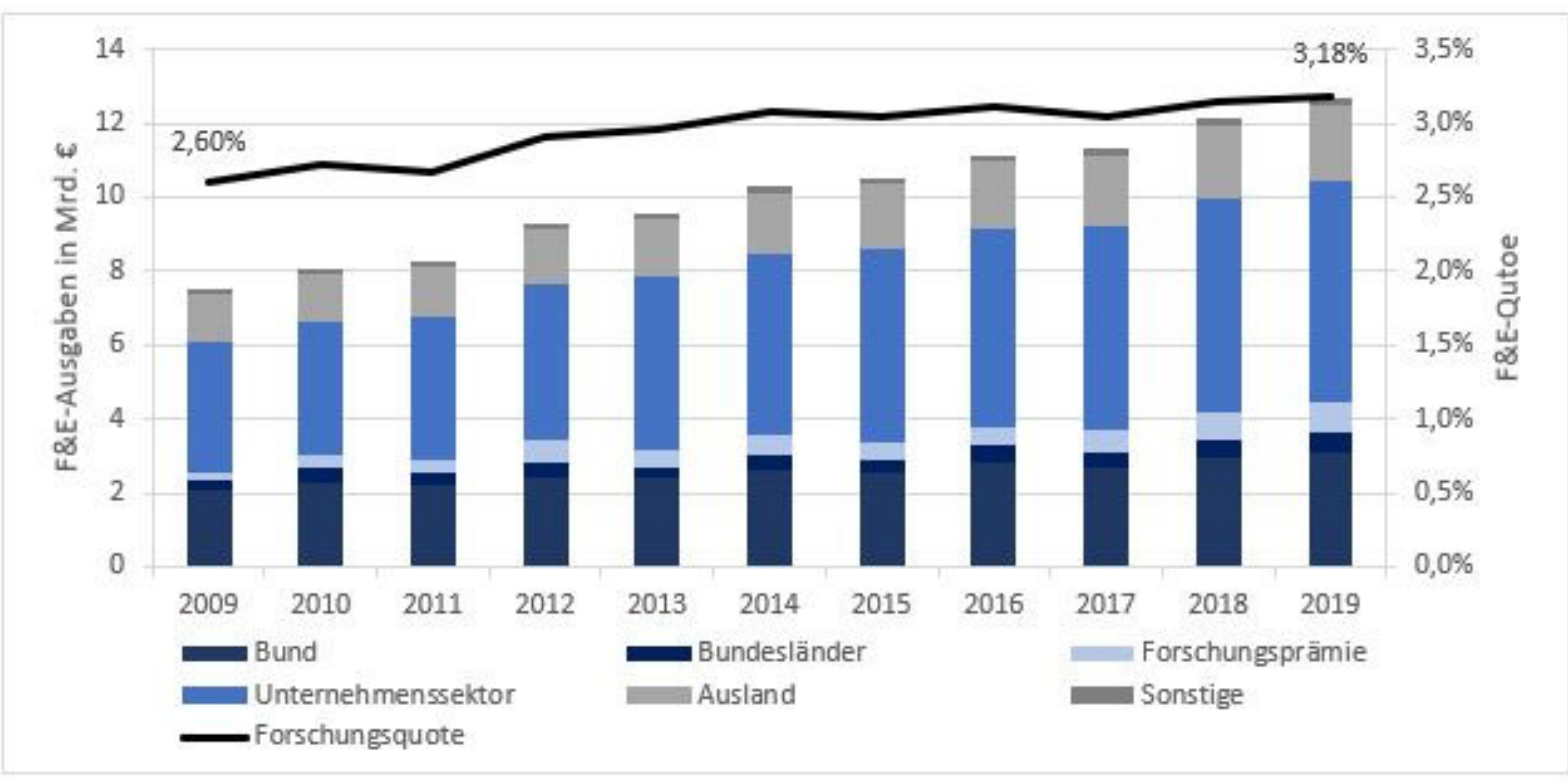

Quelle: Statistik Austria (2020).

Auf die Forschungsprämie entfielen 2019 0,76 Mrd. €, was etwa $6 \%$ der F\&E-Ausgaben entspricht. Die heimischen Unternehmen finanzierten mit 6 Mrd. € fast die Hälfte (47,6 \%) aller F\&E-Ausgaben. Aus dem Ausland flossen etwa 2 Mrd. € bzw. 15,9\%. Der größte Teil davon stammte von den heimischen Tochtergesellschaften ausländischer Konzerne. Außerdem zählen auch die Rückflüsse aus den EU-Forschungsprogrammen zu diesem Posten. Sonstige öffentliche Finanzierungen und der private gemeinnützige Sektor spielten mit gemeinsam 1,6\% nur eine untergeordnete Rolle in der Finanzierung der F\&E-Ausgaben in Österreich. 
Tabelle 1: Anteil der öffentlichen F\&E-Ausgaben am BIP, 2008 bis 2019

\begin{tabular}{|c|c|c|c|c|c|c|c|c|c|c|}
\hline 2009 & 2010 & 2011 & 2012 & 2013 & 2014 & 2015 & 2016 & 2017 & 2018 & 2019 \\
\hline $0,84 \%$ & $0,93 \%$ & $0,85 \%$ & $0,92 \%$ & $0,86 \%$ & $0,95 \%$ & $0,86 \%$ & $0,95 \%$ & $0,87 \%$ & $0,93 \%$ & $0,96 \%$ \\
\hline
\end{tabular}

Quelle: Statistik Austria, Berechnungen: WPZ Research. Anmerkung: Zwischen den öffentlich verfügbaren Daten von Eurostat (Abbildung 6) und Statistik Austria bestehen minimale Abweichungen, die vermutlich auf unterschiedliche Veröffentlichungszeitpunkte zurückzuführen sind.

Die F\&E-Ausgaben können in die drei Forschungsarten Grundlagenforschung, angewandte Forschung und experimentelle Entwicklung unterteilt werden. Die Grundlagenforschung ist dabei insbesondere für führende Innovationsnationen, die an der technologischen Grenze agieren, besonders wichtig. Viele Länder entwickeln neue Technologien nicht selbst, sondern übernehmen sie von anderen Ländern, um daraus schrittweise Verbesserungen und neue Anwendungen zu entwickeln. Innovationsführer müssen jedoch selbst die Grundlagen für neue Produkte und Anwendungen schaffen, die später revolutionären Charakter haben (Acemoglu, 2009). Diese Technologieführerschaft entsteht in der Grundlagenforschung. Österreich investierte 2017 insgesamt 1,98 Mrd. € in die Grundlagenforschung, wobei den Universitäten mit einem Anteil von 59 \% entscheidende Bedeutung zukommt (siehe Abbildung 9). Der Erfolg der Grundlagenforschung ist jedoch höchst riskant und braucht viele Jahre. Die Ergebnisse haben mehr als die kommerzielle F\&E den Charakter eines öffentlichen Gutes, deren Nutzen nicht ausschließbar ist und über SpillOver-Effekte anderen Anwendern frei zugänglich ist. In dieser Situation sind Markterlöse zur Finanzierung der Ausgaben kaum möglich. Daher betreiben die meisten Unternehmen überhaupt keine Grundlagenforschung und selbst die großen Konzerne nur eingeschränkt. Insgesamt werden immerhin $19 \%$ der Grundlagenforschung im Unternehmenssektor durchgeführt. Zum ganz überwiegenden Teil ist die Grundlagenforschung die Aufgabe der öffentlichen Hand. Sie legt damit das Fundament für die darauf aufbauende kommerzielle F\&E und richtet durch die Stärkung der Grundlagenforschung wie auch durch Schwerpunktsetzungen das Innovationssystem auf die Bewältigung aktueller gesellschaftlicher Herausforderungen aus.

Abbildung 9: F\&E-Ausgaben nach Sektoren und Forschungsarten, Mio. Euro, 2017

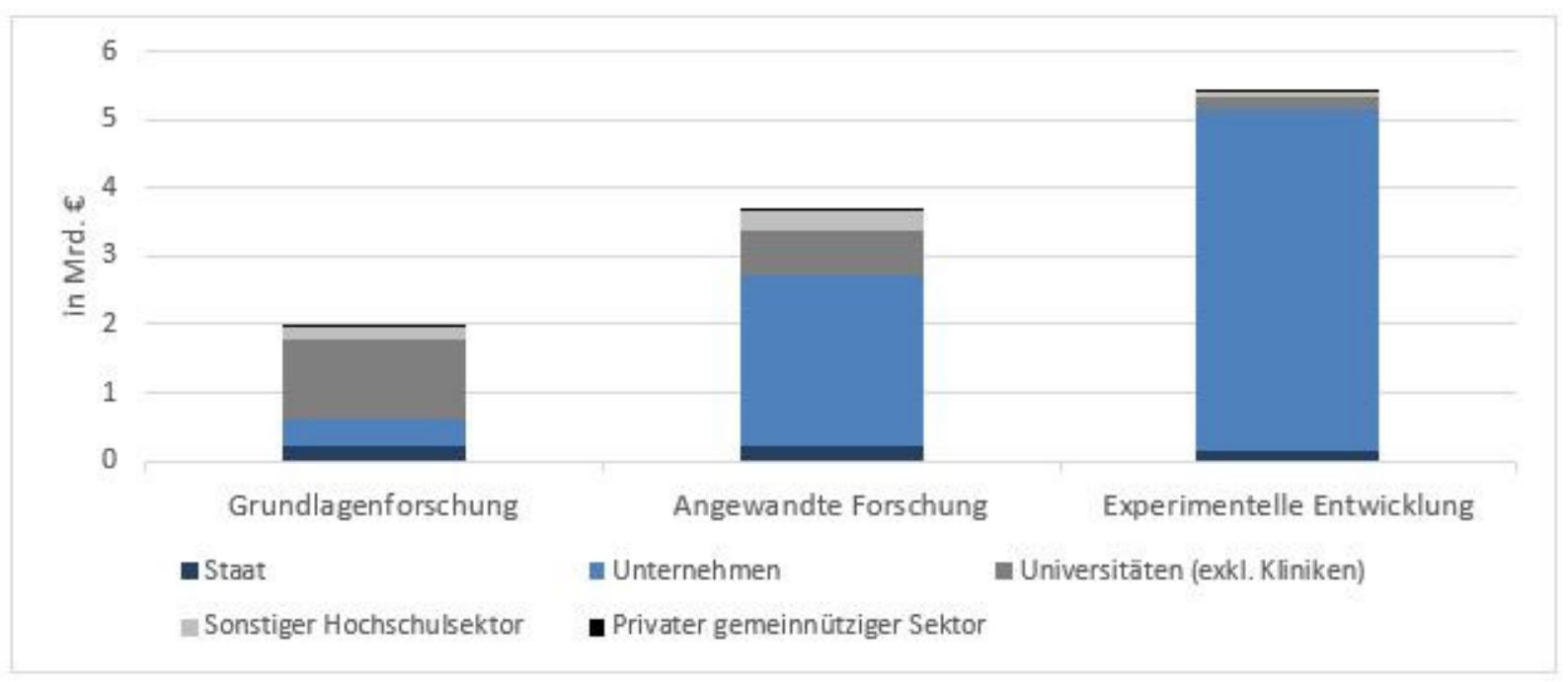

Quelle: Statistik Austria (2020). 
Auf die angewandte Forschung entfallen 3,7 Mrd.€. Sie wird zu zwei Drittel (66,7\%) vom Unternehmenssektor durchgeführt. Dieser dominiert auch in der experimentellen Entwicklung. Die Unternehmen führen $93 \%$ der gesamten experimentellen Entwicklung im Umfang von 5,4 Mrd. € durch.

Für die nächste Zukunft ist geplant, den in den letzten Jahren beschrittenen Wachstumspfad der öffentlichen F\&E-Ausgaben fortzusetzen. So budgetiert das Finanzministerium F\&E-Ausgaben des Bundes von 3,2 Mrd. € für 2020 und 3,5 Mrd. € für 2021 (BMF, 2020). Im aktuellen Regierungsprogramm ist die Ausarbeitung einer neuen FTI-Strategie mit einem Horizont bis 2030 vereinbart. Die Ziele und Maßnahmen sollen im Einklang mit der Standortstrategie und den Klimazielen stehen. Zur Operationalisierung und Umsetzung strebt die Bundesregierung einen Pakt für Forschung, Technologie und Innovation (FTI-Pakt) an, der die forschungspolitischen Schwerpunkte in einem dreijährigen Rhythmus festlegt. Ein jährlicher FTI-Gipfel soll die strategischen Schwerpunkte definieren. Zudem ist geplant, ein kooperatives Forschungsfinanzierungsmodell für Bund, Länder, Gemeinden und Private einzuführen (BKA, 2020).

Der Bund kündigt damit eine erhebliche Ausweitung von F\&E-Ausgaben an. Dagegen dürften in Folge der COVID-19-Pandemie die F\&E-Ausgaben des Unternehmenssektors deutlich zurückgehen (Friesenbichler et al., 2020; Gogola, 2020; Barlevy, 2007). Typischerweise verlaufen die Unternehmensausgaben für F\&E prozyklisch. Gründe dafür sind etwa mangelnde Liquidität und verringerte Nachfrage in Krisenzeiten. Die tiefe Rezession (Fenz und Schneider, 2020; Schiman, 2020; Bittschi et al., 2020) wird vermutlich zu ähnlich tiefen Einbrüchen bei den F\&E-Ausgaben der Unternehmen führen. Insbesondere kleine und mittlere Unternehmen, die das Rückgrat der österreichischen Wirtschaft darstellen, leiden unter Liquiditäts- und Finanzierungsproblemen in Folge der Krise. Da der Unternehmenssektor knapp die Hälfte (47,6 \%) der F\&E-Ausgaben stemmt, sind erhebliche, negative Auswirkungen auf den Innovationsstandort Österreich zu befürchten. Es wird vornehmlich am öffentlichen Sektor liegen, einem Rückgang der F\&E-Ausgaben bei den Unternehmen mit verstärkten Anreizen entgegenzuwirken, um einen Rückfall zu verhindern und die Leistungsfähigkeit des österreichischen Innovationssystems zu erhalten.

\section{Exkurs: Direkte und indirekte Forschungsförderung in Österreich}

Eine Vielzahl von Akteuren prägen das System der direkten Forschungsförderung. Das Forschungsfinanzierungsgesetz (FoFinaG) ${ }^{8}$ ändert die Rahmenbedingungen für die zentralen Forschungs- und Forschungsförderungseinrichtungen ab 2021 grundlegend. Die neuen Regelungen zielen darauf $a b$, die strategische Steuerungs- und Kontrollverantwortung der betroffenen Bundesministerien BMBWF, BMDW und BMK zu stärken und gleichzeitig die Flexibilität der Forschungs- und Forschungsförderungseinrichtungen im operativen Geschäft zu erhöhen. Das Gesetz definiert zehn Institutionen als zentrale Akteure: die Austrian Institute of Technology GmbH (AIT), das Institute of Science and Technology Austria (IST Austria), die Österreichische Akademie der Wissenschaften (ÖAW), die Silicon Austria Labs GmbH (SAL), die Austria Wirtschaftsservice GmbH (aws), die Christian Doppler Forschungsgesellschaft (CDG), der Fonds zur Förderung der wissenschaftlichen Forschung (FWF), die OeAD-GmbH (OeAD), die Österreichische Forschungsförderungsgesellschaft mbH (FFG), sowie die Ludwig Boltzmann Gesellschaft (LBG).

Ein Monitoring der Aktivitäten und strategischen Ziele erfolgt seit 2020 jährlich im Forschungs- und Technologiebericht (BMBWF et al., 2020). Den größten Teil der Forschungsförderungen des Bundes vergeben die drei großen Agenturen, FWF, FFG und aws.

\footnotetext{
8 Vgl. https://www.ris.bka.gv.at/GeltendeFassung.wxe?Abfrage=Bundesnormen\&Gesetzesnummer=20011237
} 
Der FWF ist Österreichs zentrale Einrichtung zur Förderung der Grundlagenforschung, zu dessen Aufgabenbereich die Stärkung und der Ausbau des Wissenschaftssystems zählt. Die Unterstützung von Forscherinnen und Forschern durch Einzelprojekte ist traditionell das wichtigste Instrument. Die Projektförderung erfolgt hauptsächlich bottom-up auf Basis von Anträgen, die ein internationales Peer-review Verfahren durchlaufen. Das gesamte Förderbudget des FWF betrug 2019 251,6 Mio. $€$. Etwa $40 \%$ der Förderungen gingen an den Bereich Naturwissenschaft und Technik, $37 \%$ an den Bereich Biologie und Medizin, $23 \%$ an den Bereich Geistes- und Sozialwissenschaften.

Die FFG ist die zentrale Agentur für die Förderung von angewandter Forschung, Entwicklung und Innovation. Sie ist wichtige Partnerin in der Umsetzung der Regierungsstrategien zur Stärkung des Forschungs- und Innovationsstandorts im globalen Wettbewerb und zu Digitalisierung, Klima und Energie, IPR (,Intellectual Property Rights'), sowie Open Innovation. Das Portfolio reicht von niederschwelligen Programmen, die den Einstieg von Unternehmen in eine kontinuierliche Forschungs- und Innovationstätigkeit erleichtern, bis hin zur Förderung und Finanzierung von thematischen Verbünden und Kompetenzzentren. Über die finanzielle Unterstützung hinaus bietet die FFG auch Dienstleistungen und Beratungen an. So agiert sie z.B. als Nationale Kontaktstelle für die Forschungsprogramme der Europäischen Union. Außerdem ist die FFG für die Begutachtung der Anträge zur Forschungsprämie zuständig. Im Jahr 2019 betrugen die vertraglich zugesicherten Förderungen (inklusive Haftungen und Darlehen) 618,3 Mio. €, dies entspricht einem Barwert von 493,8 Mio. €. Aktuell setzt die FFG Schwerpunkte im Bereich der Digitalisierung und Bewältigung der Klimakrise. ${ }^{9}$

Nach Angaben der FFG wären etwa vier Fünftel der geförderten Projekte ohne Förderung nicht oder nur in deutlich geringerem Ausmaß realisiert worden. Knapp die Hälfte hat innerhalb von vier Jahren direkte Auswirkungen auf die Erhaltung und Schaffung von Arbeitsplätzen. Ein Euro an Förderungen der FFG führt mittelfristig zu 10 Euro zusätzlichem Umsatz. ${ }^{10}$

Die aws ist die Förderbank des Bundes und zentrale Anlaufstelle für die Förderung von Innovation und Wachstum der Unternehmen. Durch die Vergabe von zinsgünstigen Krediten, Zuschüssen und Garantien werden Unternehmen bei der Umsetzung ihrer innovativen Projekte unterstützt, insbesondere dann, wenn sonstige Finanzierungsquellen für die erforderlichen Mittel nicht reichen oder gar nicht verfügbar sind. Ergänzend bietet die aws spezifische Informations-, Beratungs-, Service- und Dienstleistungen für angehende, bestehende und expandierende Unternehmen an. Um aktiv und zielgerichtet die internationale Wettbewerbsfähigkeit des Standortes Österreich zu stärken, definiert die aws regelmäßig ihre strategischen Schwerpunkte. Für das aktuelle Mehrjahresprogramm wurden die Schwerpunkte Digitalisierung, innovative Transformation, skalierbare Gründungen, nachhaltiges Wachstum und Internationalisierung definiert. Im Jahr 2019 lag die gesamte Finanzierungsleistung der aws bei 1,1 Mrd. €.

Wie in Kapitel 3.1 dargestellt, vergibt Österreich wie nahezu alle europäischen Staaten neben direkter auch eine indirekte F\&E-Förderung. Dies geschieht mit der Forschungsprämie, die 2002 in Höhe von $3 \%$ eingeführt und in den darauffolgenden Jahren schrittweise erhöht wurde (siehe Abbildung 10). Der Prämiensatz stieg im Jahr 2005 auf 5 \%, 2008 auf $8 \%$ und 2011 auf $10 \%$. Im Gegenzug wurde 2011 der Forschungsfreibetrag abgeschafft. Im Zuge der Steuerreform 2015/2016 wurde die Forschungsprämie auf $12 \%$ und zuletzt ab 2018 auf $14 \%$ angehoben. Parallel dazu stiegen die Ausgaben für die Forschungsprämie in den letzten Jahren stark an. Während die Steuergutschrift an Unternehmen via Forschungsprämie 2010 noch 324 Mio. $€$ betrug, lag sie im Jahr 2019 bereits bei 758 Mio. €. Für das Jahr 2020 werden 800 Mio. € erwartet, 2021825 Mio. € (BMF, 2020).

9 Vgl. https://www.ffg.at/news/ffg-foerderschwerpunkte-im-herbst-2020-digitalisierung-klimawandel-produktion
10 Vgl. https://www.ffg.at/content/zahlen-daten-fakten

Wirkung von Forschungsausgaben 


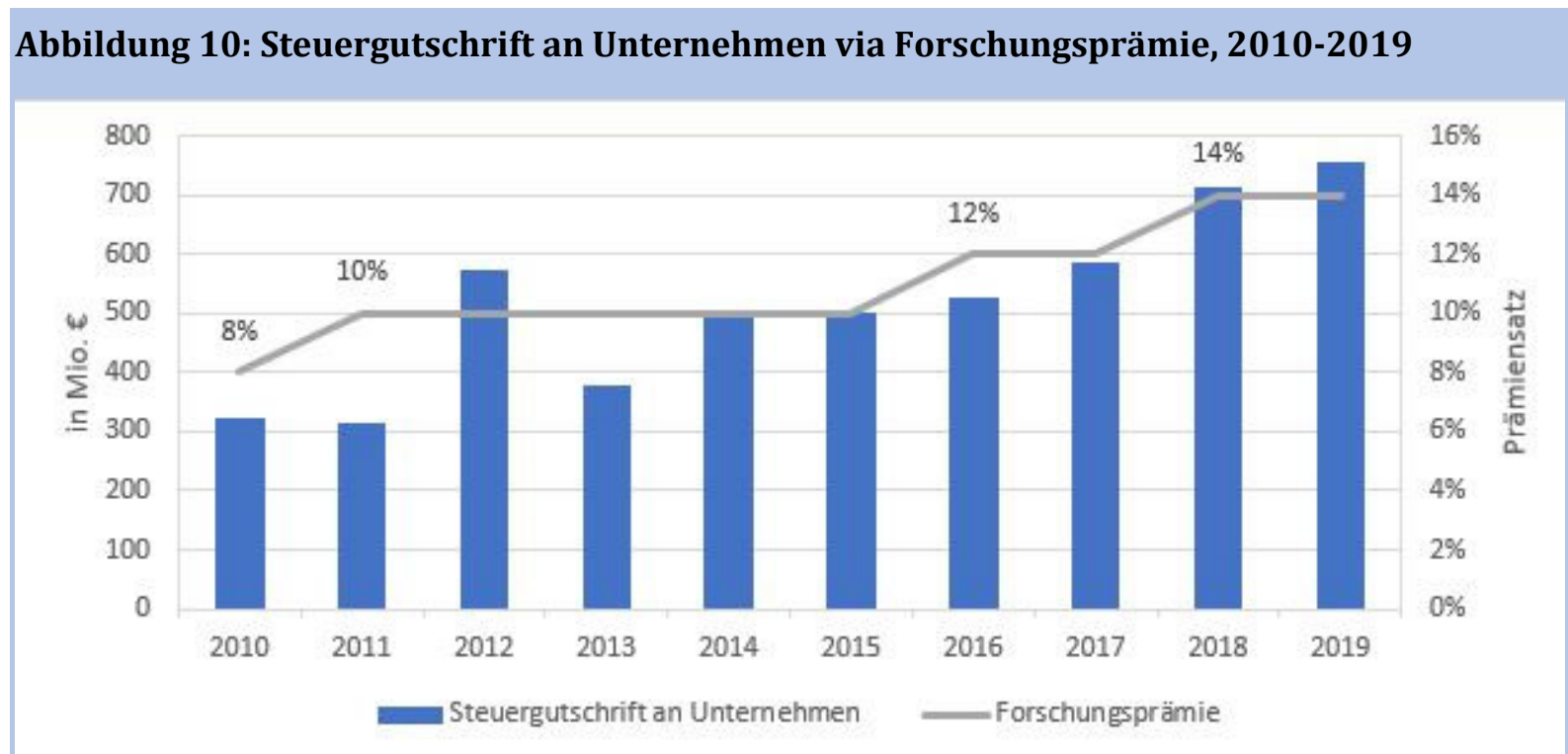

Quelle: BMF (2020).

Die Evaluierung der Forschungsprämie (Ecker et al., 2017) zeigt, dass dieses Instrument vor allem die Unternehmen unterstützt, die kontinuierlich F\&E betreiben. Die Forschungsprämie wirkt mehr in die Tiefe als in die Breite, d.h. bei bereits F\&E-intensiven Unternehmen verstärkt die Forschungsprämie die Forschungstätigkeit zusätzlich. Effekte zeigen sich dabei vor allem in Hinblick auf die Ermöglichung von mehr Investitionen in die notwendige F\&E Infrastruktur, die Übernahme eines höheren technologischen Risikos oder beschleunigende Effekte auf die Projektumsetzung. Zum Teil wurden aufgrund der Forschungsprämie F\&E-Aktivitäten nach Österreich verlagert. Eine Ausweitung der F\&E bei Unternehmen mit bislang geringer bzw. keiner F\&E bewirkt die Forschungsprämie hingegen kaum. 


\section{Wirkungen von Forschungsausgaben auf Makroebene}

Welche gesamtwirtschaftlichen Wirkungen wie etwa auf BIP, Wertschöpfung, Produktivität, Beschäftigung und andere makroökonomische Kennzahlen von Forschung und Entwicklung ausgehen, ist Inhalt der folgenden Analyse. Speziell liegt ein Fokus darauf, inwieweit sich die kurzund langfristen Effekte unterscheiden.

\subsection{Das Innovations- und Wachstumsmodell}

Das WPZ Innovationsmodell (Keuschnigg und Matt, 2020) ist ein innovationsgetriebenes Wachstumsmodell in der Klasse von DSGE-Modellen (dynamic stochastic general equilibrium) mit detaillierter Modellierung des Innovationssystems. Nach unserem Kenntnisstand gibt es in Österreich kein annähernd gleichwertiges Modell. Folgende Aspekte seien besonders betont:

- Im Kern besteht das Modell aus heterogenen Unternehmen mit unterschiedlichen Produktivitätsniveaus (Mikroebene), die einen Produktzyklus durchlaufen und aus denen mit expliziter Aggregation die makroökonomischen Aggregate hergeleitet werden (Makroebene).

- Die Heterogenität der Unternehmen entsteht aus dem Zusammenspiel von privaten F\&EInvestitionen und „technologischem Altern“. Erfolgreiche F\&E bringt eine Produktlinie mit dem höchsten Produktivitätsniveau (an der Grenze der Forschung) hervor. Nach der Einführung „altert“ die Produktlinie, sodass Know-how, Kapitalproduktivität und Profitabilität zurückfallen und die etablierten Varianten zunehmend anfälliger werden, durch neue Varianten ersetzt zu werden. Es findet ein Prozess „kreativer Zerstörung“ statt.

- Die privaten F\&E-Investitionen zielen darauf ab, (i) bereits etablierte, aber rückständige Varianten durch verbesserte Produktlinien zu ersetzen (Prozessinnovationen); und (ii) ganz neue Produktlinien einzuführen und damit neue Märkte zu erschließen (Produktinnovationen). In beiden Fällen startet die neue bzw. verbesserte Produktlinie mit dem höchst erzielbaren Produktivitätsniveau an der Grenze der Forschung.

- Die private F\&E nutzt den allgemein zugänglichen Wissensstand der Ökonomie (Stand der Forschung, Technologiegrenze), der die Ergebnisse der Grundlagenforschung und die allgemein verfügbaren Erfahrungen privater F\&E (Spill-Overs) widerspiegelt. Abhängig vom Stand der Forschung schaffen private F\&E-Investitionen neue Designs für verbesserte existierende und ganz neue Produktlinien an der Technologiegrenze, deren Marktwert von den zukünftig erzielbaren Gewinnen abhängt. Die F\&E-Intensität im Gleichgewicht wird daher vom Grenzertrag und den Grenzkosten privater F\&E getrieben.

- Die Wirtschaftspolitik kann Grenzertrag und Grenzkosten privater F\&E auf mehreren Wegen beeinflussen:

i. Eine Stärkung der Grundlagenforschung und der Initiativen für einen möglichst reibungslosen Wissenstransfer von den Universitäten und anderen Forschungseinrichtungen in die Privatwirtschaft steigert das kommerzialisierbare Know-how in der Ökonomie und schiebt die Technologiegrenze hinaus. Das hebt die Produktivität privater F\&E und steigert die Innovationsrate.

ii. F\&E-Steueranreize und F\&E-Zuschüsse (selektive Projektförderung) steigern die Innovationsrate, indem sie die privaten F\&E-Kosten der Unternehmen senken. Steueranreize fördern F\&E-Ausgaben gleichmäßig. Mit selektiver Projektförderung können zudem die besonders ertragreichen Innovationsprojekte gezielt gefördert werden. Das Modell unterscheidet zwischen „radikalen“ Produktinnovationen zur Schaffung völlig neuer Produkte und Märkte und Prozessinnovationen zur 
Verbesserung existierender Produktlinien. Eine selektive Projektförderung kann z.B. selektiv die Produktinnovationen fördern.

iii. Die Wirtschaftspolitik kann Innovation mit „Push und Pull“-Maßnahmen anregen. F\&E-Anreize sind Push-Maßnahmen. Andererseits kann sich der Ertrag privater Innovation erst über die anschließenden Kapitalinvestitionen realisieren. Ausrüstungsinvestitionen können erst erfolgen, wenn die Unternehmen über ein Geschäftsmodell bzw. Produktdesign verfügen. Sie erfolgen daher naturgemäß erst nach den F\&E-Investitionen. Steuerliche Investitionsanreize (vorzeitige Abschreibungen, Investitionsprämien etc.) sind daher Pull-Maßnahmen, welche die Profitabilität der Produktionsphase und damit den Marktwert der F\&E-Investitionen steigern, und somit ebenfalls die private Innovation stimulieren. Investitionsanreize sind insofern besonders relevant, weil sie stellvertretend auch das Potential für alle anderen Maßnahmen, wie z.B. Patentschutz und besserer Zugang zu internationalen Absatzmärkten, aufzeigen, die es den Unternehmen erlauben, das Potential ihrer Innovationen besser auszuschöpfen.

- Eine höhere F\&E-Intensität führt zu einer rascheren Verbesserung existierender Produktlinien und einer schnelleren Einführung von ganz neuen Angeboten. Indem rückständige Varianten mit geringerer Produktivität rascher durch neue Varianten an der Technologiegrenze ersetzt werden, nimmt die gesamtwirtschaftliche Produktivität auf zweifache Weise zu: (i) Ein höherer Anteil der Unternehmen (Produktlinien) befindet sich an der Technologiegrenze und verfügt damit über das höchst mögliche Produktivitätsniveau; (ii) das durchschnittliche Produktivitätsniveau der rückständigen Varianten steigt, weil die schlechtesten Varianten rascher eliminiert und durch neue ersetzt werden.

- Mit dem Start der Produktion tätigen die Unternehmen Ausrüstungsinvestitionen und heuern Beschäftigte an. Dieser Teil deckt sich im Wesentlichen mit der herkömmlichen Produktionstheorie in Makromodellen, mit dem wichtigen Unterschied, dass Kapital- und Arbeitsproduktivität vom endogen bestimmten Technologieniveau abhängen. Die private F\&E beeinflusst auf diesem Weg Ausrüstungsinvestitionen, Beschäftigung, Löhne, BIP und alle anderen makroökonomischen Kennzahlen.

- Im makroökonomischen Gleichgewicht werden die fiskalischen Kosten der Maßnahmen zur Forschungsförderung und die Rückwirkungen auf die Steuereinnahmen bzw. das Staatsbudget berechnet. Damit kann aufgezeigt werden, was die effektiven fiskalischen Kosten der Maßnahmen sind, unter Berücksichtigung von Mitnahmeeffekten und einer teilweisen Selbstfinanzierung durch zusätzliche Steuereinnahmen aufgrund der angestoßenen Wachstumseffekte.

Die Struktur des WPZ Innovationsmodells spiegelt den aktuellen Stand der Forschung in der modernen Wachstums- und Innovationstheorie wider, wie z.B. Acemoglu (2009) und Aghion und Howitt (2009). Vor allem eignet sich das Modell für Politikanalysen, wie sie z.B. Akcigit und Stantcheva (2020) systematisch besprechen. Zudem verfügt das Projektteam über ausgewiesene eigene Expertise in der Wachstumstheorie, belegt durch Publikationen in führenden Fachzeitschriften (Keuschnigg, 1994; Egger und Keuschnigg, 2015) und dem Band „Moving to the Innovation Frontier" in Keuschnigg (2016).

DSGE-Modelle sind die dominante Methode der modernen quantitativen Makroökonomie. Sie sind besser empirisch abgestützt als herkömmlich kalibrierte Modelle. Mit bayesianischen statistischen Methoden werden stochastische Schockprozesse und zentrale Verhaltensparameter so geschätzt, dass das Modell die vergangene Entwicklung als Schwankungen um einen stationären Wachstumspfad herum möglichst gut nachvollziehen kann. Danach können durch Änderung von 
Politikinstrumenten, wie z.B. Steuersätze und Subventionsraten, die Auswirkungen von wirtschaftspolitischen Maßnahmen quantifiziert werden.

Simulationsanalysen mit dem DSGE-Modell können die kurz- und langfristigen Folgen der Forschungsförderung quantifizieren, die im Wachstumsgleichgewicht der österreichischen Wirtschaft inhärent zusammenhängen. Die langfristigen Effekte im stationären Gleichgewicht ergeben sich erst nach Abschluss aller Anpassungsvorgänge im Übergangspfad. Es sollen die unterschiedlichen kurz- und langfristigen Effekte herausgearbeitet werden. Wie lange dauert es, bis etwa die Hälfte der langfristigen Auswirkungen realisiert ist (Halbwertszeit der Anpassung)? Die Darstellung dieses Zeithorizonts sollte für die Wirtschaftspolitik von großem Interesse sein.

Speziell sollen in der folgenden Analyse zwei Fragen beantwortet werden: Erstens, wie wirksam ist die öffentliche Forschungsförderung? Konkret quantifizieren wir, welcher BIP-Zuwachs pro Euro an Budgetmitteln für öffentliche Forschungsausgaben (Forschungsprämie, direkte Subventionen, Ausgaben für öffentliche F\&E) ausgelöst wird. Selbstverständlich zeigen wir auch die Auswirkungen auf die anderen wichtigen makroökonomischen Kennzahlen auf. Zweitens, wieviel des Wachstums hängt ursächlich von der Wirksamkeit des Innovationssystems ab? Konkret berechnen wir, um wieviel das Wachstum geringer ausfallen würde, wenn alle Innovationskanäle einfrieren, also weder private noch öffentliche F\&E-Ausgaben sich verändern können. Dieses Szenario vertieft die Studie der EUKommission (2017), welche in der restriktivsten Definition von F\&E-Investitionen Wachstumsbeiträge zwischen $17 \%-30 \%$ des BIP-Wachstums ermittelt, und in der breitesten Definition einen Beitrag von bis zu zwei Dritteln des Wachstums. Einschränkend ist hinzuzufügen, dass die an der EU-Studie beteiligten Forscherinnen und Forscher nicht über ein konsistentes, makroökonomisches Innovationsmodell verfügen, sondern die unabhängigen Ergebnisse verschiedener empirischer Studien zusammengefügt haben. Umso wichtiger ist es, ähnliche Berechnungen mit einem empirisch fundierten Innovationsmodell anzustellen, welches die makroökonomischen Zusammenhänge und Rückwirkungen auf die öffentlichen Haushalte konsequent berücksichtigt.

\subsection{Langfristige Wirkungen öffentlicher Forschungsausgaben}

Die Ausgaben für öffentlich-finanzierte F\&E und für öffentliche Forschungsförderung stößt Wachstum an und hebt das langfristige BIP auf ein höheres Niveau. Die öffentliche Forschungsförderung enthält steuerliche F\&E-Förderung mittels der Forschungsprämie sowie direkte Subventionen, wie z.B. die Ausgaben für die Programme der FFG. Wenn mit höherer Innovationsneigung die Produktivität zunimmt, schwenkt die Wirtschaft auf einen höheren Wachstumspfad ein. Löhne, Profitabilität der Investitionen, Kapitalbildung und Arbeitsangebot steigen an. Das BIP nimmt auf zweifache Weise zu. Die höhere Produktivität ermöglicht beim selben Faktoreinsatz einen höheren Output. Die ausgelösten Investitionen und die höhere Beschäftigung führen zu zusätzlichen BIP-Zuwächsen, die den direkten Effekt verstärken. Die steigenden verfügbaren Einkommen steigern den Konsum als Maß für den Wohlstand.

Wie hoch sind die BIP-Zuwächse pro Euro an eingesetzten Budgetmitteln? Um diese Frage zu beantworten, skalieren wir zunächst alle öffentlichen Forschungsausgaben mit einem einheitlichen Faktor hoch, so dass die Struktur der Forschungsausgaben unverändert bleibt. Konkret wählen wir einen Impuls von zusätzlichen öffentlichen Forschungsausgaben von $0.25 \%$ des BIP. Dieser Betrag ist weder Bestandteil des Regierungsprogramms noch eine Empfehlung der Wissenschaft, und könnte daher grösser oder kleiner ausfallen. Die quantitativen Auswirkungen fallen naturgemäß umso höher aus, je grösser die Budgetsteigerungen angesetzt werden. Um diesen Unterschieden Rechnung zu tragen, berechnen wir die Ergebnisse pro Euro an zusätzlichen öffentlichen Forschungsausgaben. 
Abbildung 11 zeigt den langfristigen BIP-Zuwachs pro Euro an zusätzlichen öffentlichen F\&EAusgaben. Die langfristigen Effekte treten erst nach Abschluss aller Anpassungsvorgänge ein. Dabei ist zu berücksichtigen, dass die zusätzlichen Forschungsausgaben eine Gegenfinanzierung mit höheren Steuern (oder auch geringeren Ausgaben) benötigen. Wir nehmen dabei an, dass die Struktur der Besteuerung unverändert bleibt, und skalieren alle Steuersätze mit einem einheitlichen Faktor hoch, bis das öffentliche Budget bei konstanter Schuldenquote vollständig ausfinanziert ist. Für sich genommen wirken höhere Steuern negativ auf Investitionen in Ausrüstungen und F\&E und auf die Beschäftigung. Solange die Forschungsausgaben produktiv sind, können höhere Steuern den BIPZuwachs mindern, aber nicht umkehren. Unsere beste Schätzung für den Nettoeffekt ist: 1 Euro an F\&E-Ausgaben löst einen langfristigen BIP-Zuwachs von knapp 6 Euro aus.

\section{Abbildung 11: Langfristiger BIP-Zuwachs pro Euro öffentlicher Forschungsausgaben}

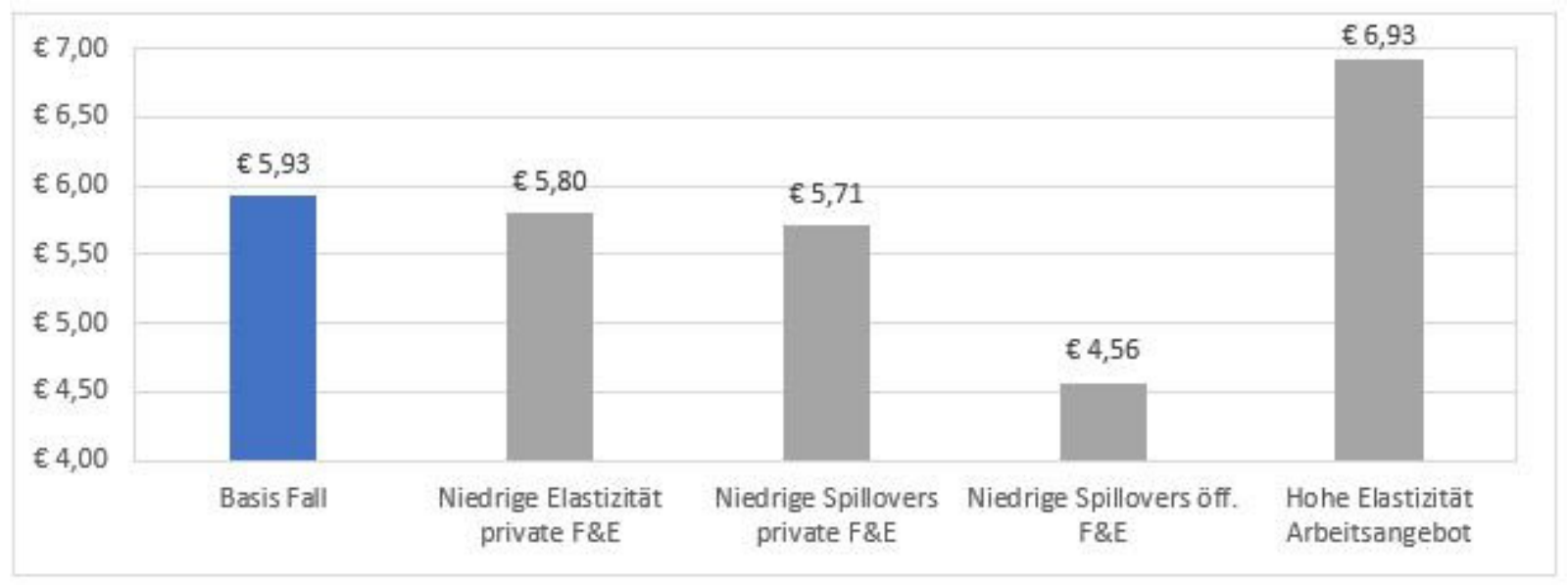

Berechnung und Darstellung: WPZ.

Der blaue Balken ist der Basisfall, also unsere beste Schätzung. Wie in jedem quantitativen Modell gibt es eine gewisse Unsicherheit über die exakten Parameterwerte, welche die Anpassungen der Wirtschaft treiben. Beispielsweise sind die Auswirkungen umso grösser, je produktiver die öffentlichen Ausgaben für Grundlagenforschung sind und je wirksamer die öffentliche Forschungsförderung die private F\&E anregen kann. Das Modell ist so parametrisiert, dass unter sonst konstanten Bedingungen die privaten F\&E-Ausgaben um 1,5 \% zunehmen (Semielastizität), wenn die Subventionsquote um einen Prozentpunkt steigt. Dieser Wert entspricht typischen Schätzungen mit Firmendaten, wobei die verfügbaren Schätzungen durchaus erheblich streuen, also teilweise niedriger liegen, aber auch deutlich höhere Werte von bis zu $2 \%$ rechtfertigen. Der zweite Balken zeigt, dass mit einer niedrigeren Semielastizität von 1,3 \% der langfristige Gesamteffekt von 5,93 Euro im Basisfall auf 5,8 Euro fällt. Das Ergebnis ist also relativ robust bezüglich einer Variation der Semielastizität privater F\&E-Ausgaben.

Die Forschung weist zudem auf deutlich positive externe Effekte privater F\&E-Ausgaben hin. Demnach tragen private F\&E-Investitionen auch zum gemeinsamen Wissensbestand in der Wirtschaft bei, der von allen Unternehmen ohne Rivalität und ohne Kosten nutzbar ist und den Erfolg der privaten F\&E-Investitionen stärkt. Je mehr F\&E alle anderen Unternehmen betreiben, desto erfolgreicher ist die F\&E in jedem einzelnen Unternehmen, und umgekehrt. Im Basisfall steigt - unter sonst konstanten Bedingungen - der allgemein nutzbare Wissensbestand (die Technologiegrenze) um $0,4 \%$, wenn die privaten F\&E-Investitionen um $1 \%$ zunehmen. Diese (partielle) Elastizität ist eigenständig mit bayesianischen Methoden modellkonsistent geschätzt. Wenn im Zuge der Sensitivitätsanalyse dieser Wert auf 0,3\% reduziert wird und damit die externen Erträge in diesem 
Ausmaß geringer ausfallen, dann fällt auch der BIP-Zuwachs von 5,8 auf 5,7 Euro (dritter Balken in Abbildung 11). Auch diese Sensitivität ist gering.

Ein zentraler Bestandteil öffentlicher Forschungsausgaben sind die Aufwendungen für Grundlagenforschung an Universitäten, außeruniversitären Forschungseinrichtungen und Fachhochschulen, die den allgemein nutzbaren Wissensbestand steigern und auf diesem Weg wie eine Vorleistung die private F\&E erleichtern. Wie oben schätzen wir diese Elastizität mit 0,4 , so dass der kommerzialisierbare Wissensstock (die Technologiegrenze) um 0,4\% zunimmt, wenn die öffentlichen Ausgaben für Grundlagenforschung um $1 \%$ ansteigen. Wenn die Produktivität der öffentlichen Ausgaben abnimmt und die Elastizität auf 0,3 sinkt, dann sind diese Ausgaben weniger wirksam. Nach dem vierten Balken in Abbildung 11 sinkt dann der BIP-Zuwachs von 5,9 Euro im Basisfall auf 4,6 Euro ab. Das ist eine erheblich größere Sensitivität. Daraus ergeben sich zwei Schlussfolgerungen. Erstens gilt es, besonderen Aufwand in die Schätzung dieses Parameters zu stecken und die damit bestimmte Wirksamkeit der öffentlichen F\&E-Ausgaben mit den Ergebnissen der unabhängigen empirischen Forschung abzugleichen. Zweitens misst diese Elastizität, wie sehr öffentliche Ausgaben für Grundlagenforschung private Innovationen stimulieren können. Diese Wirksamkeit ist mit Maßnahmen der Wirtschaftspolitik beeinflussbar, welche die Barrieren für den Technologietransfer in die Privatwirtschaft und für eine raschere Kommerzialisierung der Grundlagenforschung abbauen.

Die privaten und öffentlichen Forschungsausgaben sind die Quelle für Produktivitätssteigerungen. Eine zunehmende Produktivität steigert die Rentabilität und stößt Investitionen, Kapitalbildung und Beschäftigungsnachfrage an. Ein Teil der Produktivitätsgewinne ermöglicht höhere Reallöhne, welche die Anreize für Erwerbsbeteiligung und das gesamtwirtschaftliche Arbeitsangebot stärken. Mehr Beschäftigung und mehr Investitionen steigern das BIP. Diese Mechanismen multiplizieren die Effekte des Innovationssystems. Abbildung 11 illustriert diesen Zusammenhang am Beispiel der Arbeitsangebotsreaktion. Je elastischer das Arbeitsangebot auf Lohnanreize reagiert, desto höher fällt der Hebeleffekt auf die Wirkung von Forschungsausgaben aus. Der Basisfall beruht auf einer Lohnelastizität des Arbeitsangebots von 0,25, d.h. wenn die Reallöhne um $10 \%$ steigen, nimmt das Arbeitsangebot um 2,5\% zu. Dieser Wert entspricht den typischen Ergebnissen der empirischen Arbeitsmarktforschung, allerdings mit erheblicher Streuung der Ergebnisse. Der letzte Balken berechnet die Wirkungen des Forschungsimpulses bei einer höheren Elastizität von 0,33 (ein Reallohnzuwachs von $10 \%$ würde das Arbeitsangebot um 3,3\% ausweiten). Der Hebeleffekt ist bemerkenswert. In diesem Fall ermitteln wir einen BIP-Zuwachs von fast 7 Euro pro Euro an zusätzlichen Forschungsausgaben. Die Wirkung von Forschungsausgaben hängt also auch sehr davon ab, wie elastisch Investitionen und Beschäftigung auf die erzielten Produktivitätsgewinne reagieren. Dieser Zusammenhang wird nachfolgend anhand der Quantifizierung der Wachstumsbeiträge der Innovation sehr deutlich (vgl. Abbildung 14 im nächsten Abschnitt).

Das BIP ist nur ein summarisches Maß für die Wirkungen der Forschungsausgaben. Tabelle 2 zeigt die langfristigen Auswirkungen nach Abschluss aller Anpassungsvorgänge in größerem Detail, die sich aus öffentlichen Mehrausgaben für Forschung in der Höhe von 0,25 \% des BIPs ergeben (Impuls). Die Staatsschuldenquote bleibt konstant und ist nur der Vollständigkeit halber aufgeführt, zum Vergleich mit den nachfolgenden Szenarien. Sie beträgt $240 \%$ des BIPs pro Quartal, das entspricht $60 \%$ des jährlichen BIPs. Wir gehen davon aus, dass die momentanen Verschuldungsquoten temporäre Abweichungen vom langfristigen Wert nach den Maastricht Vorgaben darstellen.

Das Steuersystem ist in der gebotenen Einfachheit dargestellt und generiert ein Steueraufkommen, welches den Staatsanteil von fast $50 \%$ des BIPs finanzieren muss. In der Ausgangssituation beträgt der Grenzsteuersatz auf alle Formen von Einkommen (auf Arbeit und Kapital) $42 \%$. Wegen der Aushöhlung der Bemessungsgrundlage ist die Durchschnittsbelastung wesentlich geringer. Der Effektivsteuersatz auf den Konsum beträgt $18 \%$. Wenn höhere Steuereinnahmen notwendig werden, 
werden alle Steuersätze proportional angehoben, ohne die Steuerstruktur zu verändern. Weil der Impuls nur 0,25 \% des BIPs ausmacht, sind auch die absoluten Auswirkungen relativ klein.

Tabelle 2: Langfristige Effekte öffentlicher F\&E-Ausgaben

\begin{tabular}{|l|c|c|c|c|c|}
\hline & Basis & $\begin{array}{c}\text { Fall 1 } \\
\text { Hohe Elastizität } \\
\text { privater F\&E }\end{array}$ & $\begin{array}{c}\text { Fall 2 } \\
\text { Niedrige Spill- } \\
\text { Overs privater } \\
\text { F\&E }\end{array}$ & $\begin{array}{c}\text { Fall 3 } \\
\text { niedrige Spill- } \\
\text { Overs } \\
\text { öffentlicher F\&E }\end{array}$ & $\begin{array}{c}\text { Fall 4 } \\
\text { hohe } \\
\text { Arbeitsangebots } \\
\text {-elastizität }\end{array}$ \\
\hline Steuersätze & 41,835 & 41,835 & 41,842 & 41,941 & 41,733 \\
\hline Schuldenquote & 240,000 & 240,000 & 240,000 & 240,000 & 240,000 \\
\hline BIP & 1,532 & 1,496 & 1,476 & 1,180 & 1,790 \\
\hline $\begin{array}{l}\text { Faktorproduktivitä } \\
\text { t }\end{array}$ & 0,747 & 0,731 & 0,725 & 0,608 & 0,755 \\
\hline Kapitalstock & 1,612 & 1,572 & 1,547 & 1,199 & 1,930 \\
\hline Beschäftigung & 0,392 & 0,381 & 0,373 & 0,274 & 0,606 \\
\hline Technologiegrenze & 10,329 & 10,230 & 9,948 & 7,945 & 10,375 \\
\hline $\begin{array}{l}\text { Private F\&E } \\
\text { Ausgaben }\end{array}$ & 4,743 & 4,420 & 4,706 & 4,511 & 4,895 \\
\hline Neue Produkte & 2,375 & 2,214 & 2,357 & 2,261 & 2,450 \\
\hline Reallohn & 1,113 & 1,089 & 1,077 & 0,855 & 1,151 \\
\hline
\end{tabular}

Berechnung und Darstellung: WPZ.

Anmerkung: In der Ausgangssituation beträgt der Steuersatz $42 \%$. Die ersten beiden Zeilen zeigen Absolutwerte, die folgenden Zeilen Veränderungen in \%.

Das Wirtschaftswachstum speist sich aus der Zunahme des Arbeitsvolumens, dem Aufbau des produktiven Kapitalstocks und der Zunahme der Faktorproduktivität. Auslöser dafür sind die höheren privaten und öffentlichen F\&E-Investitionen, welche die Technologiegrenze, d.h. den allgemein nutzbaren Wissensbestand der Wirtschaft, um 10,3 \% hinausschieben. Die privaten F\&EAusgaben fallen im langfristigen Gleichgewicht um 4,7 \% höher aus und führen zu mehr Prozess- und Produktinnovationen. Das vermehrte Know-how mündet schlussendlich in einer um 0,7 \% höheren Faktorproduktivität, erkauft mit Mehrausgaben von $0,25 \%$ des BIPs. Eine höhere Faktorproduktivität steigert den Ertrag von Arbeit und Kapital. Das zeigt sich in einem Anstieg des Reallohns um 1,1\%, der die Arbeitsanreize stärkt und die Beschäftigung um 0,4\% steigen lässt. Ohne diesen Beschäftigungszuwachs wäre die Lohnsteigerung sogar noch grösser. Der Anstieg der Kapitalrendite stößt Investitionen und Kapitalbildung an. Im langfristigen Wachstumsgleichgewicht steigt der Kapitalstock so stark an, um 1,6 \% im Vergleich zur Ausgangssituation, dass die Kapitalrendite gerade wieder auf den international vorgegebenen, marktüblichen Zins fällt.

Mehr Innovation durch private und öffentliche F\&E steigert die totale Faktorproduktivität. Mit demselben Faktoreinsatz kann damit ein höheres BIP erwirtschaftet werden. Die Folgewirkungen auf Kapitalbildung und Arbeitsvolumen multiplizieren die direkten Auswirkungen der Innovation auf die Faktorproduktivität und lassen die BIP-Zuwächse umso grösser ausfallen. Die zusätzlichen Forschungsausgaben in der Höhe von 0,25 \% des BIPs lösen einen BIP-Zuwachs von 1,5 \% aus. Dies entspricht dem BIP-Zuwachs von etwa 6 Euro pro Euro an Forschungsausgaben $(1,5 / 0,25)$, wie es dem Basisfall von Abbildung 11 entspricht. Bemerkenswert ist, dass im langfristigen Gleichgewicht die Steuersätze in etwa konstant bleiben bzw. sogar ganz leicht gegenüber der Ausgangssituation fallen (41,8\% statt $42 \%)$. Die Ausgaben wären demnach langfristig selbstfinanzierend. Dieses ungewöhnliche Ergebnis wird allerdings erst in der nachfolgenden Gegenüberstellung der kurz- und langfristigen Ergebnisse verständlich. Die Fälle 1-4 in Tabelle 2 zeigen qualitativ dieselben 
Wirkungen. Die konkreten Zahlen variieren etwas, je nach den Sensitivitäten, die vorhin in Abbildung 11 bereits besprochen wurden.

\subsection{Dynamische Auswirkungen}

Kapitalbildung und Aufbau des Wissensstocks (Know-how) durch Grundlagenforschung und private Innovation sind langsame Prozesse. Es dauert viele Jahre, bis sich die vollen Auswirkungen einstellen. Letzten Endes sind zusätzliche Forschungsausgaben wie eine Investition in die Zukunft zu betrachten: Die höheren Forschungsausgaben fallen sofort an, aber die Einkommensgewinne schlagen erst nach vielen Jahren voll zu Buche. Die Regierung müsste also sofort die Steuersätze anheben, was die gegenwärtige Wirtschaftsentwicklung schwächt und die Einkommenszuwächse hinausschiebt. Wenn dann nach einer langen Anpassungsphase der BIP-Zuwachs in vollem Ausmaß da ist, sprudeln die Steuerquellen. Die zukünftigen Steuersätze können umso niedriger sein.

Dieser Mechanismus wirkt wie eine intergenerative Umverteilung, welche die künftigen Generationen begünstigt und die heute lebenden Generationen belastet. Um die Vorteile der Innovation gleichmäßiger zu verteilen, kann der Staat die fiskalischen Lasten zusätzlicher Forschungsausgaben mit dem Einsatz der Staatsverschuldung über die Zeit strecken. Durch teilweise Schuldenfinanzierung kann er prinzipiell eine allzu starke Anhebung der Steuersätze heute vermeiden, muss aber dann in Hinkunft etwas höhere Steuersätze akzeptieren, um die aufgelaufene Staatsschuld zu bedienen. Ein wichtiger Zweck der Staatsverschuldung ist die zeitliche Streckung einmaliger fiskalischer Sonderlasten, um die Steuersätze über die Zeit zu glätten. Einschränkend sei hinzugefügt, dass eine solche Schuldenpolitik eine solide Finanzpolitik voraussetzt. Wenn schon aus vielen anderen Gründen die Staatsschuld ausufert und an die Grenzen der fiskalischen Tragbarkeit stößt, ist eine solche intergenerativ ausgleichende Finanzpolitik kaum mehr möglich.

Abbildung 12 illustriert die Dynamik, die mit höheren Forschungsausgaben ausgelöst wird, und zeigt gleichzeitig den Unterschied, den eine ausgleichende Schuldenpolitik zur Glättung der Steuersätze über die Zeit ausmachen kann. Das BIP ist auf 100 normalisiert. So können privater Konsum und andere Nachfragekomponenten oder die Faktoreinkommen in Prozent des BIP verstanden werden. Die horizontalen Linien zeigen das stationäre Wachstumsgleichgewicht der Ökonomie in der Ausgangslage. Das exogene Trendwachstum ist dabei herausgerechnet, so dass das wachstumsbereinigte BIP in der Ausgangslage konstant bleibt. In absoluten Größen würde es mit der Trendwachstumsrate von ca. 1,5\% jährlich wachsen. Ein Ausbau der öffentlichen Ausgaben für Forschung im Umfang von 1/4 Prozentpunkt des BIPs löst nach Tabelle 2 einen langfristigen BIPZuwachs von etwa 1,5 \% aus, das ist nach Abbildung 11 etwa das Sechsfache des Budgetaufwands. In Abbildung 12 kommt daher das (trendbereinigte) BIP nach Abschluss der Anpassungsvorgänge auf einem 1,5 \% höheren Niveau zu liegen, d.h. der BIP-Index steigt von 100 auf 101,5 an.

Die öffentliche Forschungsförderung hebt das BIP auf einen höheren Wachstumspfad. Damit es dorthin gelangt, sind in einer ersten Phase die Wachstumsraten viel höher als die Trendrate von 1,5 \% jährlich. Wenn dann mit einem dauerhaft höheren öffentlichen Aufwand der Wachstumszugewinn realisiert ist, flacht die BIP-Kurve wieder ab, sprich die Wachstumsraten fallen langsam wieder auf die Trendwachstumsrate zurück. Steil geneigte Kurven zeigen Phasen hoher Wachstumsraten, die das BIP und andere Größen auf ein langfristig höheres Niveau heben. Abbildung 13 zeigt die Unterschiede relativ zur Trendwachstumsrate von 1,5\% in ausgewählten Jahren nach Beginn des dauerhaften Impulses. Nach einer geringfügigen Verlangsamung in den ersten drei Jahren übersteigt das Wachstum über einen langen Zeitraum den Trend. Die grauen Balken zeigen die Anpassung bei sofortiger Gegenfinanzierung mit höheren Steuern. Im ersten Jahr fällt die Wachstumsrate des BIP von 1,5\% auf 1,46\%, das entspricht einer Differenz von -0,04 Prozentpunkten. Nach drei Jahren wird die Differenz positiv. Das BIP beginnt schneller als im Trend zu wachsen. Im Jahr 10, also nach 40 Quartalen, ist der Zugewinn mit einem differentiellen Wachstum von 0,05 Prozentpunkten am 
größten. Die positiven Wirkungen halten jedoch sehr lange an. Da der Impuls mit Mehrausgaben von $0,25 \%$ des BIPs sehr klein ist, sind auch die Auswirkungen sehr klein.

\section{Abbildung 12: Dynamische Wirkungen öffentlicher F\&E-Ausgaben}
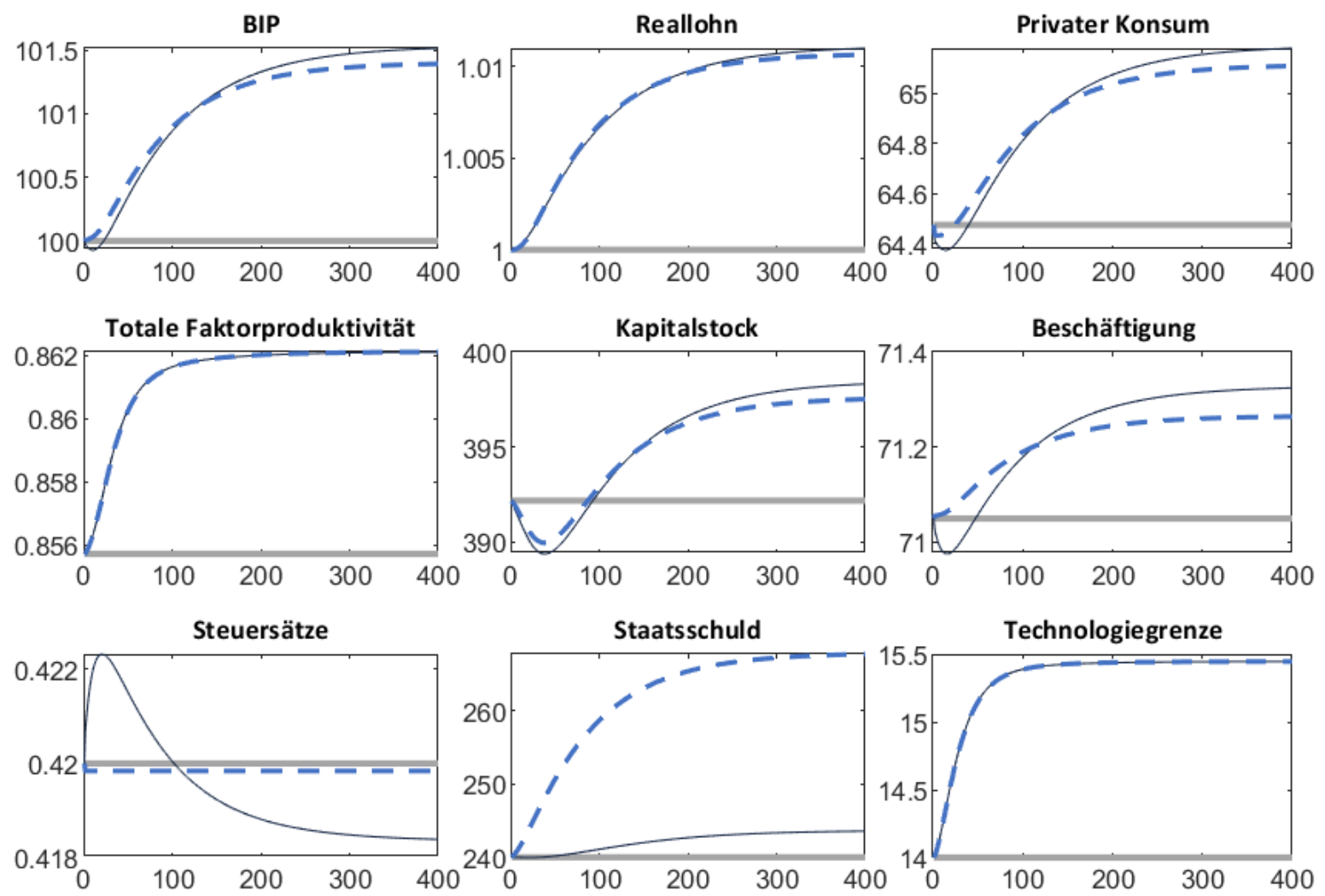

Berechnung und Darstellung: WPZ.

Anmerkung: Die horizontale Achse zeigt Quartale nach Beginn der öffentlichen Mehrausgaben. Die durchgezogenen Kurven zeigen Anpassungen mit periodischer Finanzierung, die gestrichelten Kurven die Anpassung mit Glättung der Steuersätze durch Einsatz der Staatsschuld.

Abbildung 12 verfolgt die Zeitreihen über 400 Quartale, das entspricht einem Zeitraum von 100 Jahren (Abbildung 13 weist dieselbe Anpassung in Jahren aus). Dann sind die Anpassungsprozesse zwar nicht exakt, aber fast vollständig abgeschlossen. Die Anpassung dauert also sehr lange, länger als bei anderen Politikszenarien. ${ }^{11}$ Neben der visuellen Veranschaulichung in Abbildung 12 lässt sich die Anpassungsgeschwindigkeit mit der Halbwertszeit angeben. Für das Szenario ohne fiskalischen Ausgleich (d.h., ohne Steuerglättung) beträgt die Halbwertszeit der BIP Anpassung 22 Jahre (88 Quartale). So viel Zeit braucht es, bis die Hälfte des gesamten langfristigen BIP-Zuwachses von 1,5\%

11 In der endogenen Wachstumstheorie steigt nicht nur das Niveau, sondern auch die Trendwachstumsrate auf ein langfristig höheres Niveau, also z.B. von 1,5 \% auf 1,7 \% oder ähnlich. In diesem Fall würden die Wachstumspfade in Abbildung 12 nicht mehr flach werden. Die Phase höheren Wachstums würde überhaupt nicht mehr aufhören. Langanhaltende Anpassungsvorgänge sind ein Schritt in diese Richtung. Die empirische Evidenz spricht allerdings eher von abnehmenden Grenzerträgen der Innovation, so dass die Ökonomie nach langanhaltenden Anpassungszeiträumen wieder auf die Trendwachstumsrate zurückfällt. Längere Anpassungspfade deuten daher an, dass das Verhalten der Modellökonomie sich den Gesetzen der endogenen Wachstumstheorie annähert. 
des BIPs (im Vergleich zum Status quo) realisiert ist. Drei Viertel der Anpassung sind nach 37 Jahren (148 Quartalen) vollzogen. Mit anderen Worten, F\&E-Investitionen haben sehr langanhaltende, eben nachhaltige Auswirkungen auf das Wirtschaftswachstum. Die positiven Effekte wirken sehr viel länger als bei anderen Initiativen der Wirtschaftspolitik. Dieses Muster schlägt auch auf die meisten anderen Kennzahlen wie privater Konsum, Beschäftigung oder Kapitalstock durch.

\section{Abbildung 13: Differentielle, jährliche Wachstumsraten des BIP, in Prozentpunkten}

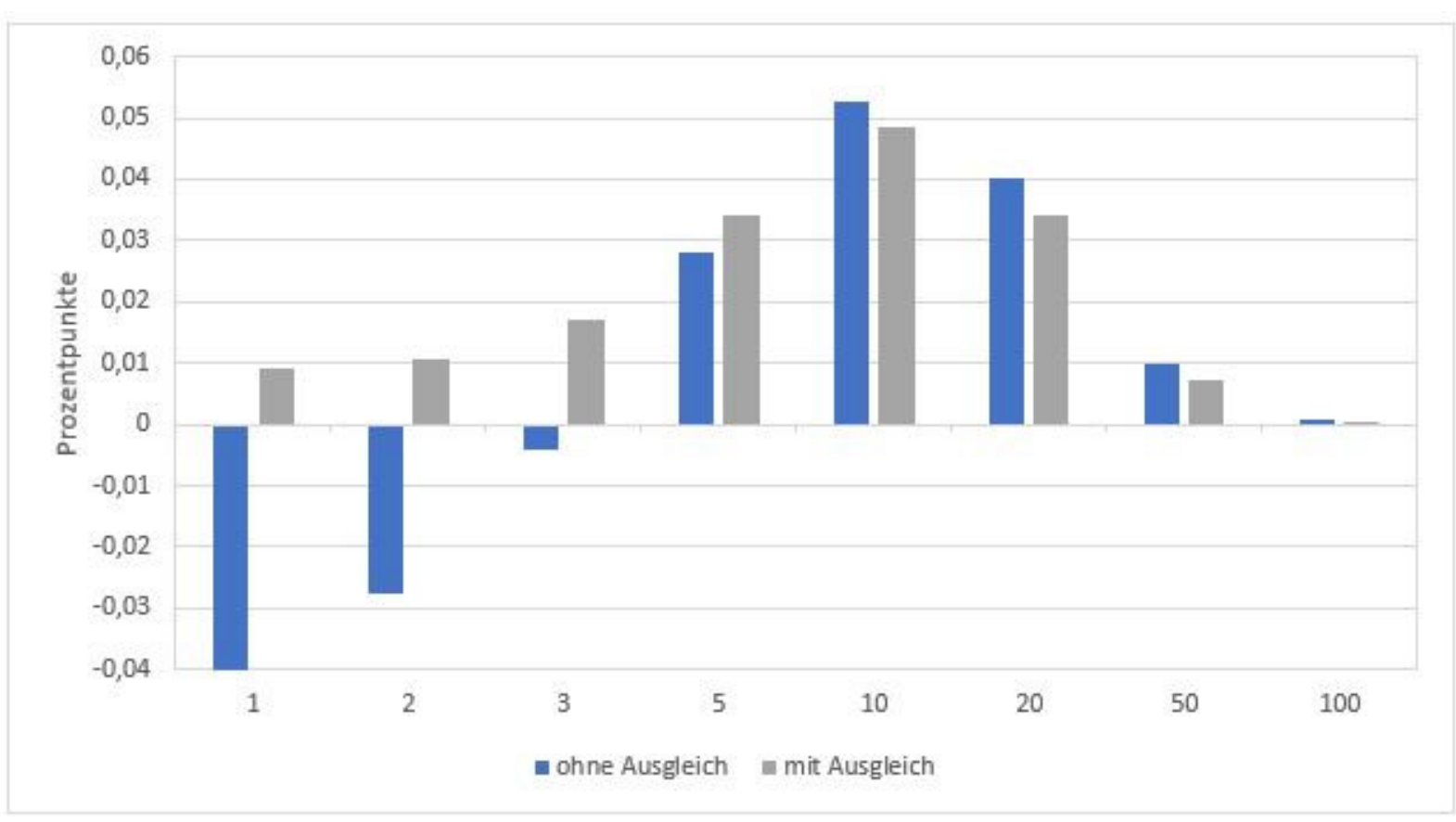

Berechnung und Darstellung: WPZ.

Anmerkung: Die Balken zeigen die Differenz zur Trendwachstumsrate von 1.5 \% in ausgewählten Jahren nach Beginn der dauerhaften F\&E-Mehrausgaben. Die blauen (grauen) Balken zeigen die Effekte ohne (mit) fiskalischem Ausgleich durch Staatsverschuldung zur Glättung der Steuersätze.

Die Innovationspolitik braucht einen langen Atem. Demnach besteht folgende Herausforderung für die Wirtschaftspolitik: Die Budgetkosten der öffentlichen Forschungsförderung sind sofort da, aber die Wachstumsgewinne kommen nur langsam und schlagen erst in der ferneren Zukunft voll zu Buche. Wie bei jeder Investition muss also der Staat zuerst Ausgaben tätigen, bevor die zukünftigen Erträge in Form von Einkommenssteigerungen zufließen. Dieser Aspekt kommt im ersten Szenario (schwarze, durchgezogene Linien in Abbildung 12) sehr klar zum Ausdruck. Dieses Szenario unterstellt, dass der Staat die langfristige Staatsschuldenquote nach den Maastricht-Kriterien bei $60 \%$ des BIPs hält. Abweichungen davon, ausgelöst z.B. durch einen sofortigen Anstieg der öffentlichen F\&E-Förderung, müssen also durch eine Konsolidierungspolitik wieder beseitigt werden, wobei eine gewisse zeitliche Streckung der Konsolidierung zulässig ist. Wenn das BIP langfristig um 1,5\% höher ausfällt, kann auch die Staatsschuld um diesen Prozentsatz steigen, damit die Schuldenquote unverändert bleibt. Daher kommt es in Abbildung 12 mit dem BIP-Anstieg auch zu einem Anstieg der Staatsverschuldung.

In unserem ersten Szenario unterstellen wir, dass die fiskalische Konsolidierung mit einer Anhebung aller Steuersätze erfolgt, ohne die Steuerstruktur zu verändern. Die Herausforderung für die Finanzpolitik zeigt sich in Abbildung 12 sehr deutlich. Die Steuersätze müssen zuerst steigen, um den sofortigen Anstieg der Ausgaben zu finanzieren, anstatt in die Verschuldung gehen zu lassen. Der Anstieg mag gering erscheinen, aber er betrifft alle Steuern und entspricht daher annähernd der 
Steuerquote (in Prozent des BIPs). Nachdem die zusätzlichen fiskalischen Ausgaben 0,25 \% des BIPs ausmachen, muss auch die Steuerquote zunächst um etwa 0,25 Prozentpunkte steigen. Die höheren Steuersätze beeinträchtigen in der ersten Phase die Investitions- und Arbeitsanreize, so dass es zunächst zu einem geringfügigen Rückgang von Beschäftigung und Kapitalstock kommt. Daher geht auch das BIP geringfügig zurück, bevor es dann stark ansteigt.

Sobald dann die Innovationsimpulse nennenswert auf die Faktorproduktivität durchschlagen, werden die Investitionen rentabler. Das löst einen Boom in den Ausrüstungsinvestitionen aus. Die zunehmenden Produktivitätsgewinne ermöglichen höhere Reallöhne, welche Arbeitsanreize und Beschäftigung stärken. Es setzt ein starkes BIP-Wachstum ein, das sowohl von Produktivitätssteigerungen als auch von mehr Beschäftigung und Kapitaleinsatz getragen wird. Der Anstieg von Lohn- und Gewinneinkommen und des Konsums weitet die Steuerbasis aus und spült starke zunehmende Steuereinnahmen in das Budget. Nach der ersten Anpassungsphase mit höheren Steuersätzen können diese wieder fallen, ohne den Pfad der Staatsverschuldung zu beinträchtigen, bis sie nach etwa 25 Jahren (100 Quartalen) sogar unter das ursprüngliche Niveau zu liegen kommen. Auf die lange Sicht ist also in diesem Szenario der sofortigen Gegenfinanzierung die öffentliche Forschungsförderung sogar selbstfinanzierend, aber eben erst nach vielen Jahren. Die Selbstfinanzierung wird plausibel, wenn man sich das Ergebnis von Abbildung 11 vergegenwärtigt, wonach ein Euro an Forschungsausgaben langfristig etwa 6 Euro an BIP-Zuwachs auslösen.

Die gestrichelten Kurven in Abbildung 12 illustrieren, wie der Staat durch Einsatz der Staatsverschuldung die Steuersätze über die Zeit konstant halten kann. Dabei muss das gewählte Niveau in allen Perioden ausreichend hohe Steuereinnahmen erzielen, um alle Ausgaben vollständig zu finanzieren. Indem der Staat in der ersten Phase die höheren Forschungsausgaben mit Schulden finanziert, kann er den Anstieg der Steuersätze vermeiden. Allerdings müssen dann in Zukunft die Steuersätze auf dem gleichen Niveau bleiben und können nicht mehr fallen, weil die Zinsbelastung der in der ersten Phase aufgelaufenen höheren Staatsschuld zu finanzieren ist. Die Abbildung zeigt, dass der endogen ermittelte Steuersatz praktisch nicht mehr vom Ausgangsniveau abweicht, also weder steigt noch fällt, und die Forschungsausgaben gerade selbstfinanzierend sind. Das ist ein Zufallsergebnis. Die Staatsschuld liegt am Ende um etwa 7,5\% des jährlichen BIPs höher, das entspricht 30 Prozentpunkten des Quartals-BIPs.

Wenn mit dieser Steuerglättungspolitik die Steuersätze in der ersten Phase geringer und in Zukunft höher liegen als im Basisszenario mit sofortiger Gegenfinanzierung, dann fördert dies die kurzfristige und belastet die langfristige Wirtschaftsentwicklung. Weil sich damit kurzfristig negative Effekte auf die Beschäftigung vermeiden lassen, steigt das BIP zunächst langsam und dann schneller an, ohne je zu fallen (siehe auch die grauen Balken in Abbildung 13). Die anderen Variablen folgen mehr oder weniger demselben Muster. Insbesondere verlagert die Schuldenpolitik die zukünftigen Beschäftigungsgewinne in die Gegenwart. Dasselbe gilt für den privaten Konsum. Die Drehung der Beschäftigungsreaktionen hebt - kaum merkbar - die heutigen Reallöhne zulasten zukünftiger Löhne an. Der Kapitalstock sinkt in einer ersten Phase weniger stark, kann aber dann in Zukunft auch weniger stark zunehmen, weil dann die Steuersätze vergleichsweise höher sind und damit die Investitionsanreize geringer ausfallen. Auch diese Anpassungen tragen zur Reduktion des kurzfristigen BIP-Verlusts in der ersten Phase bei.

Höhere Forschungsausgaben haben langanhaltende, wachstumsbelebende Auswirkungen. Die positiven Effekte schlagen erst nach einer langen Phase der Anpassung voll zu Buche, während die Ausgaben sofort ansteigen müssen, um den Wachstumsprozess anzustoßen. Damit werden staatliche Forschungsausgaben zu einer Investition in die Zukunft. Wie bei jeder Investition müssen zunächst die Kosten geschultert werden, bevor die Erträge fließen. Diese Kosten müssen finanziert werden, entweder mit höheren Steuern (bzw. Verzicht auf andere Ausgaben) oder mit Staatsschuld. Auch private Investitionen verursachen zunächst Investitionskosten, bevor die ausgelösten Produktivitätszuwächse die Gewinne steigern. Die Wahl zwischen sofortiger Steuerfinanzierung oder 
Aufnahme von neuen Staatsschulden hat allerdings wesentliche intergenerative Verteilungswirkungen. Wenn bei sofortiger Steuerfinanzierung die heutigen Generationen auf Einkommen und Konsum verzichten, können künftige Generationen umso mehr profitieren. Umgekehrt können mit dem Einsatz der Staatsverschuldung die Kosten über die Zeit gestreckt werden, um die volkswirtschaftlichen Erträge und Kosten der Forschungsausgaben gleichmäßiger auf heutige und künftige Generationen zu verteilen. Künftige Generationen profitieren von höheren Einkommen in der Zukunft, und müssen dabei mit der Bedienung der höheren Staatsschuld anteilig an den höheren Kosten mitzahlen.

\subsection{Wachstumsbeiträge der Innovation}

Der Staat steigert sowohl mit öffentlicher Forschungsförderung als auch mit den Ausgaben für F\&E die Rendite privater Innovation. Die Unternehmen investieren aber auch ohne öffentliche Förderung in F\&E, wenn sie mit den damit erzielten Qualitätssteigerungen und neuen Produktlinien ihre künftigen Gewinne steigern können. Sie bauen mit Innovation ihre Wettbewerbsfähigkeit aus und erobern größere Marktanteile. Die privaten F\&E-Investitionen nehmen zu, wenn der Barwert der künftigen Gewinnsteigerungen die zusätzlichen F\&E-Kosten übersteigt. Innovation steigert die Faktorproduktivität, so dass mit demselben Faktoreinsatz mehr Output produziert wird. Wenn Arbeits- und Kapitalproduktivität zunehmen, erwirtschaften Arbeitseinsatz und Kapitalbildung einen höheren Ertrag. Eine höhere Faktorproduktivität löst in der Folge zusätzliche Investitionen und mehr Beschäftigung aus, die den Wachstumsbeitrag der Innovation zusätzlich steigern. Mit anderen Worten: Gäbe es keine Innovation, dann würden nicht nur die Faktorproduktivität leiden, sondern auch Kapitalbildung und Arbeitseinsatz fallen.

Wieviel vom Wachstum ist unter Berücksichtigung dieser Zusammenhänge auf Innovation zurückzuführen? Um diese Frage zu beantworten, ist wieder ein Impuls notwendig, der die Entwicklung anstößt. Um ein solches Wachstumsszenario zu konstruieren, orientieren wir uns an vergangenen Entwicklungen. Von 2005 bis 2019 ist das BIP in Österreich um durchschnittlich 1,44 \% jährlich gewachsen. Im selben Zeitraum hat das Arbeitsvolumen um 0,4\% jährlich zugenommen. Dieser Anstieg spiegelt im Wesentlichen die Zunahme der Erwerbsbevölkerung wider, während in den letzten 15 Jahren die geleisteten Arbeitsstunden pro Kopf der erwerbsfähigen Bevölkerung fast unverändert geblieben sind. Daher halten wir im Modellszenario die Erwerbsquote als summarisches Maß für das Arbeitsangebot konstant. Ein wichtiger, wirtschaftspolitisch und daher modellexogen bestimmter Wachstumsfaktor ist die Entwicklung der öffentlichen Ausgaben für F\&E und für die Forschungsförderung. Von 2005-2019 sind die öffentlichen Ausgaben (exklusive Forschungsprämie) um durchschnittlich 2,74\% pro Jahr angestiegen, also deutlich stärker als das reale BIP. Sodann wurde in den letzten 10 Jahren die Forschungsprämie schrittweise von $8 \%$ auf $14 \%$ ausgebaut. Das sind deutliche Innovationsimpulse, die wesentlich zum vergangenen Wachstum beigetragen haben, und noch erhebliche Zeit nachwirken sollten.

Im nächsten Schritt leiten wir ein Wachstumsszenario für die Zukunft ab, welches im Wesentlichen diesem Muster entspricht, und zerlegen dieses dann in die Wachstumskomponenten, um den Effekt der Innovation zu isolieren. Konkret dimensionieren wir das Szenario derart, dass ein langfristiger BIP-Zuwachs von 15,4 \% resultiert, das entspricht dem kumulativen Wachstum der letzten 10 Jahre, während das Arbeitsangebot pro Kopf wie in der Vergangenheit konstant bleibt. Diese Effekte werden mit folgenden Impulsen erreicht:

- Das Erwerbspotential steigt langfristig um 3,9 \%, das entspricht der kumulativen Zunahme über die letzten 10 Jahre.

- Die öffentlichen Ausgaben für Grundlagenforschung nehmen um total $31 \%$ zu, das entspricht der kumulativen Zunahme der letzten 10 Jahre. 
- Die Forschungsprämie wird um 6 Prozentpunkte angehoben, das entspricht der schrittweisen Erhöhung der Prämie von $8 \%$ auf $14 \%$ in den letzten 10 Jahren.

- Die exogene Komponente der totalen Faktorproduktivität erhöht sich um insgesamt 5,3 \%. Diese Veränderung ist so berechnet, dass alles in allem ein langfristiger BIP-Zuwachs von 15,4\% resultiert (im Umfang gleich der kumulativen Zunahme der letzten 10 Jahre).

- Das Arbeitsangebot pro Kopf bleibt konstant, wie es in den letzten 10 Jahren beobachtet wurde. Da Innovation und Kapitalakkumulation die Löhne steigern, würde damit das Arbeitsangebot zunehmen. Daher wird eine abnehmende Bereitschaft zur Erwerbsbeteiligung (Präferenzparameter) berechnet, welche diesen Effekt gerade neutralisiert.

Das Wachstumsszenario löst damit per Konstruktion einen langfristigen BIP-Zuwachs von 15,4 \% aus, wie Abbildung 14 im ersten Balken anzeigt. Die Impulse sind damit etwa zehn Mal so stark wie in den Szenarien der beiden vorausgehenden Abschnitte.

\section{Abbildung 14: Langfristige Wachstumsbeiträge der Innovation}

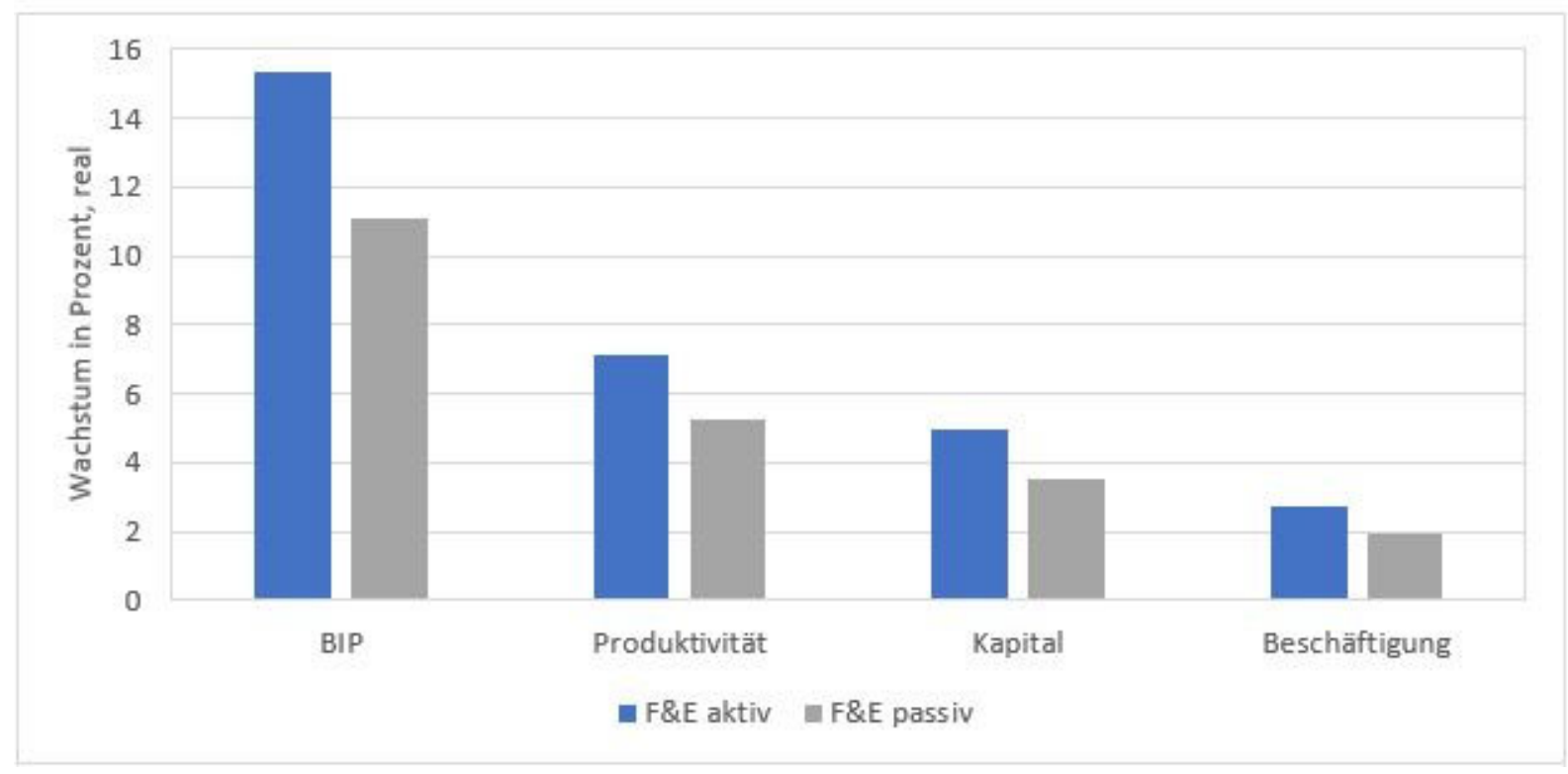

Berechnung und Darstellung: WPZ.

Anmerkung: Die jeweils letzten drei Balken summieren sich näherungsweise auf den ersten Balken auf.

Wir betrachten zuerst die blauen Balken: Der kumulative BIP-Zuwachs speist sich aus den Wachstumsbeiträgen der Produktivitätssteigerungen (zweiter Balken), der Kapitalbildung durch Investitionen (dritter Balken) und der Beschäftigung (vierter Balken). Diese drei Quellen addieren sich annähernd auf den Gesamteffekt im ersten Balken auf. Mit 7,1 \% steuert die Zunahme der totalen Faktorproduktivität mit Abstand den größten Teil, beinahe die Hälfte, zum BIP-Wachstum bei. Der Anstieg der Faktorproduktivität selbst besteht aus einer exogenen Komponente (5,3\% wie oben), die mit den Produktivitätssteigerungen aufgrund mehr privater Innovation gehebelt wird (zusätzliche 1,8 Prozentpunkte). Der zweitwichtigste Beitrag entfällt auf die Kapitalbildung. Der Kapitalstock nimmt langfristig um etwa 16,6\% zu (siehe Tabelle 3). Dieser Anstieg muss mit dem Wertschöpfungsanteil des Kapitals von $30 \%$ multipliziert werden, um den Wachstumsbeitrag der Kapitalbildung zu ermitteln. Dieser beträgt somit $5 \%$. Schließlich entfallen 2,7 \% des kumulativen BIP-Zuwachses auf die Zunahme der Beschäftigung. Diese speist sich sowohl aus dem Anstieg des exogenen Erwerbspotentials als auch dem Anstieg des Arbeitsangebots pro Kopf. Die 
Erwerbsbeteiligung bleibt jedoch wegen der kompensierenden Abnahme der Erwerbsneigung per Konstruktion unverändert, so dass der Beschäftigungszuwachs allein die Zunahme der Erwerbsbevölkerung widerspiegelt und damit $3.9 \%$ beträgt (siehe Szenario oben). Bei einem Wertschöpfungsanteil von $70 \%$ ergibt dies einen Wachstumsbeitrag zum BIP von $2.7 \%$.

Wie groß würde im gleichen Szenario das BIP-Wachstum ausfallen, wenn das Innovationssystem ausgeschaltet bliebe und nicht reagieren könnte? Damit wären die F\&E-Investitionen der Unternehmen eingefroren und könnten das Know-how und die Produktivität nicht steigern. Die öffentlichen Innovationsanreize würden keinen Effekt auf private F\&E-Investitionen entfalten. Sie wären wirkungslos. Ebenso wirkungslos wären die Steigerungen des Budgets für die Grundlagenforschung. Sie könnten keinen anregenden Effekt mehr auf private Innovation entfalten. Jede Möglichkeit, über Innovationen die totale Faktorproduktivität zu steigern, wäre ausgeschaltet. Das Wachstum würde dann nur mehr von der Ausweitung des physischen Arbeitsangebots und der rohen Kapitalbildung gespeist. Dass Innovation auch die Produktivität des Arbeitseinsatzes und des Kapitalstocks steigert, bliebe außen vor. Der geringere Anstieg der Faktorproduktivität würde in der Folge auch die Investitions- und Beschäftigungsanreize mindern, weil bei geringerer Produktivität Investition und Beschäftigung weniger rentabel sind.

Dasselbe Wachstumsszenario kann damit nur eine wesentlich geringere BIP-Zunahme bewirken. Das zeigen die rechts angeordneten, grauen Balken. Der langfristige BIP-Zuwachs in Abbildung 14 beträgt nur mehr 11,1 \% anstatt 15,4\%. Die Differenz von 4,3 Prozentpunkten bedeutet, dass die Reaktion des Innovationssystems den langfristigen BIP-Zuwachs um beinahe zwei Fünftel anhebt, d.h. um genau $39 \%(=100 * 4,3 / 11,1)$ des Wachstums ohne Innovation. Umgekehrt betrachtet sind vom gesamten BIP-Zuwachs $28 \%(=100 * 4,3 / 15,4)$ auf Innovation zurückzuführen und der Rest auf die anderen Wachstumsquellen. Der differentielle Wachstumsbeitrag der Innovation speist sich aus drei Quellen: Erstens steigern die privaten F\&E-Investitionen, angeregt um die Effekte der Grundlagenforschung, direkt die Faktorproduktivität. Derselbe Faktoreinsatz ermöglicht ein höheres BIP. Der differentielle Effekt des Innovationssystems auf die Faktorproduktivität beträgt 1,8 Prozentpunkte. Der größte Teil der Zunahme der Faktorproduktivität speist sich aus exogenen Quellen und beträgt allein schon 5,3\%. Die Reaktion des Innovationssystems lässt also die Faktorproduktivität um 7,1\% anstatt 5,3\% wachsen (siehe Tabelle 3). Dieser differentielle Effekt von 1,8 Prozentpunkten stärkt die Investitions- und Beschäftigungsanreize und multipliziert damit die Wachstumsbeiträge von Kapital und Arbeit. Alle drei Wachstumsquellen fließen stärker und akkumulieren sich zu einem differentiellen Gesamteffekt von 4,3 Prozentpunkten mehr Wachstum.

Damit sind im betrachteten Wachstumsszenario etwa $28 \%$ des langfristigen BIP-Zuwachses ursächlich auf die Reaktion des Innovationssystems zurückzuführen. Diesen Effekt muss man zudem im Verhältnis zu den Aufwendungen sehen. Setzt man die Wachstumsbeiträge ins Verhältnis zum Ressourceneinsatz, ergibt sich ein durchaus eindrückliches Bild. Die F\&E-Quote beträgt nur knapp $3 \%$ des BIP! Die Investitionen in den Kapitalstock betragen ein Vielfaches davon. Die Wertschöpfungsanteile des Kapitals und der Arbeit betragen $30 \%$ bzw. $70 \%$ des BIPs. Der Beitrag der Innovation zum BIP-Zuwachs von $28 \%$ geht mit einem relativ geringen Ressourcenaufwand einher. Umgekehrt bedeutet dies, dass pro Euro an Ressourcenaufwand ein sehr starker Effekt auf das BIP erzielt wird. Einen Hinweis darauf ergibt Abbildung 11, wonach 1 Euro an öffentlicher F\&EFörderung einen BIP Zuwachs von 6 Euro auslöst.

Eine EU-Studie aus 2017 (Europäische Kommission, 2017) kommt zu ähnlich hohen Wachstumsbeiträgen. Die Ergebnisse dieser Studie beruhen auf einer Synthese von einer Reihe unabhängiger empirischer Schätzungen über Einzelaspekte der Innovation. Anders als in modellgestützten Analysen bleiben gesamtwirtschaftliche Restriktionen und Zusammenhänge einschließlich der Rückkoppelungen zwischen öffentlichen Finanzen und gesamtwirtschaftlichem Wachstum unberücksichtigt. 
Annähernd vergleichbar mit der Schätzung in der vorliegenden Arbeit ist beispielsweise das Ergebnis, dass Forschung und Innovation die Arbeitsproduktivität im EU-Durchschnitt um 17\%, aber in Irland um etwa $20 \%$ gesteigert hätten (Europäische Kommission, 2017, S. 3). In unserer modellgestützten Studie hebt die endogene Reaktion des Innovationssystems die Zunahme der Faktorproduktivität von 5,3\% auf 7,1\%. Der differentielle Effekt von 1,8 Prozentpunkten bedeutet, dass etwa $25 \%(1,8 / 7,1)$ des Produktivitätswachstums von den F\&E-Effekten verursacht werden. Allerdings sind die totale Faktorproduktivität und die Arbeitsproduktivität nicht ganz identisch. Angesichts der üblichen Sensitivitäten von Modellanalysen genauso wie von ökonometrischen Schätzungen sind diese Größenordnungen vergleichbar.

\section{Tabelle 3: Langfristige Wachstumsbeiträge und makroökonomische Auswirkungen}

\begin{tabular}{|l|c|c|}
\hline & mit F\&E & ohne F\&E \\
\hline Steuersätze & 39,98 & 40,8 \\
\hline Schuldenquote & 240,00 & 240,00 \\
\hline BIP & 15,37 & 11,11 \\
\hline Faktorproduktivität & 7,09 & 5,25 \\
\hline Kapitalstock & 16,55 & 11,76 \\
\hline Beschäftigung & 3,87 & 2,80 \\
\hline Technologiegrenze & 18,69 & 0,00 \\
\hline Private F\&E Ausgaben & 24,21 & 0,00 \\
\hline Neue Produkte & 11,25 & 0,00 \\
\hline Reallohn & 10,86 & 7,92 \\
\hline
\end{tabular}

Berechnung und Darstellung: WPZ.

Anmerkung: Die Ergebnisse sind mit Abbildung $14 \mathrm{zu}$ vergleichen.

Um ein detaillierteres Bild zu zeichnen, spaltet Tabelle 3 die langfristigen Effekte des Szenarios weiter auf. Die grundsätzlichen Effekte wurden bereits im Zusammenhang mit Abbildung 14 besprochen. Tabelle 3 zeigt, wie das Innovationssystem reagiert. Die privaten F\&E-Investitionen steigen um etwa 24 \%. Diese bewirken einen um 11,3 \% höheren Zufluss von neuen Produkten und beschleunigen die Prozessinnovationen, die zu einer höheren Qualität der etablierten Produktlinien führen. Dieser mächtige Anstieg ist direkt auf die stufenweise Anhebung der Forschungsprämie sowie dem kumulativen Zuwachs der öffentlichen Mehrausgaben für F\&E zurückzuführen. Sowohl die Mehrausgaben für F\&E als auch die positiven externen Effekte der privaten F\&E-Ausgaben schieben die Technologiegrenze, also den allgemein nutzbaren Wissensbestand in der Ökonomie, hinaus. Die Zunahme des Wissensbestands um 18,7 \% ist ebenfalls ein zentraler Treiber privater F\&E. Auf diesem Weg beflügelt die Grundlagenforschung wie eine Vorleistung bzw. ein Technologietransfer die privaten F\&E-Investitionen. Wie die zweite Spalte zeigt, würden diese Zuwächse nicht stattfinden, wenn das Innovationssystem ausgeschaltet bliebe. Die innovationsgetriebenen Produktivitätssteigerungen ermöglichen starke Lohnzuwächse um 10,9\%, die um 3 Prozentpunkte höher ausfallen als ohne Innovation.

Abbildung 15 zeigt die dynamischen Auswirkungen des Wachstumsszenarios. Die Anpassung verläuft sehr langsam, aus denselben Gründen, die bereits in Abschnitt 4.3 diskutiert wurden. Im Wachstumsszenario dieses Abschnitts kommt als Besonderheit hinzu, dass der Anstieg der Erwerbsbevölkerung ein langsamer, demographischer Prozess ist, der die Anpassung zusätzlich hinauszögert. Nach Abschluss aller Anpassungsvorgänge werden die Kurven in Abbildung 15 vollständig flach, so dass die makroökonomischen Kennzahlen dieselben Endwerte annehmen, wie sie in Tabelle 3 beschrieben wurden. Mit ähnlich langsamer Geschwindigkeit tut sich eine immer grösser werdende Lücke zwischen den Werten mit und ohne F\&E auf. In diesem Szenario bleibt die 
Staatsschuldenquote annähernd konstant, so dass das Volumen der Staatsschuld annäherungsweise parallel mit dem BIP wachsen kann. Ähnlich wie in Abschnitt 4.3 könnte eine ausgleichende Verschuldungspolitik gewählt werden, welche die Steuersätze über die Zeit konstant hält. Das könnte kurzfristig negative Auswirkungen der Steuerfinanzierung weitgehend beseitigen.

\section{Abbildung 15: Dynamische Wachstumsbeiträge der Innovation}
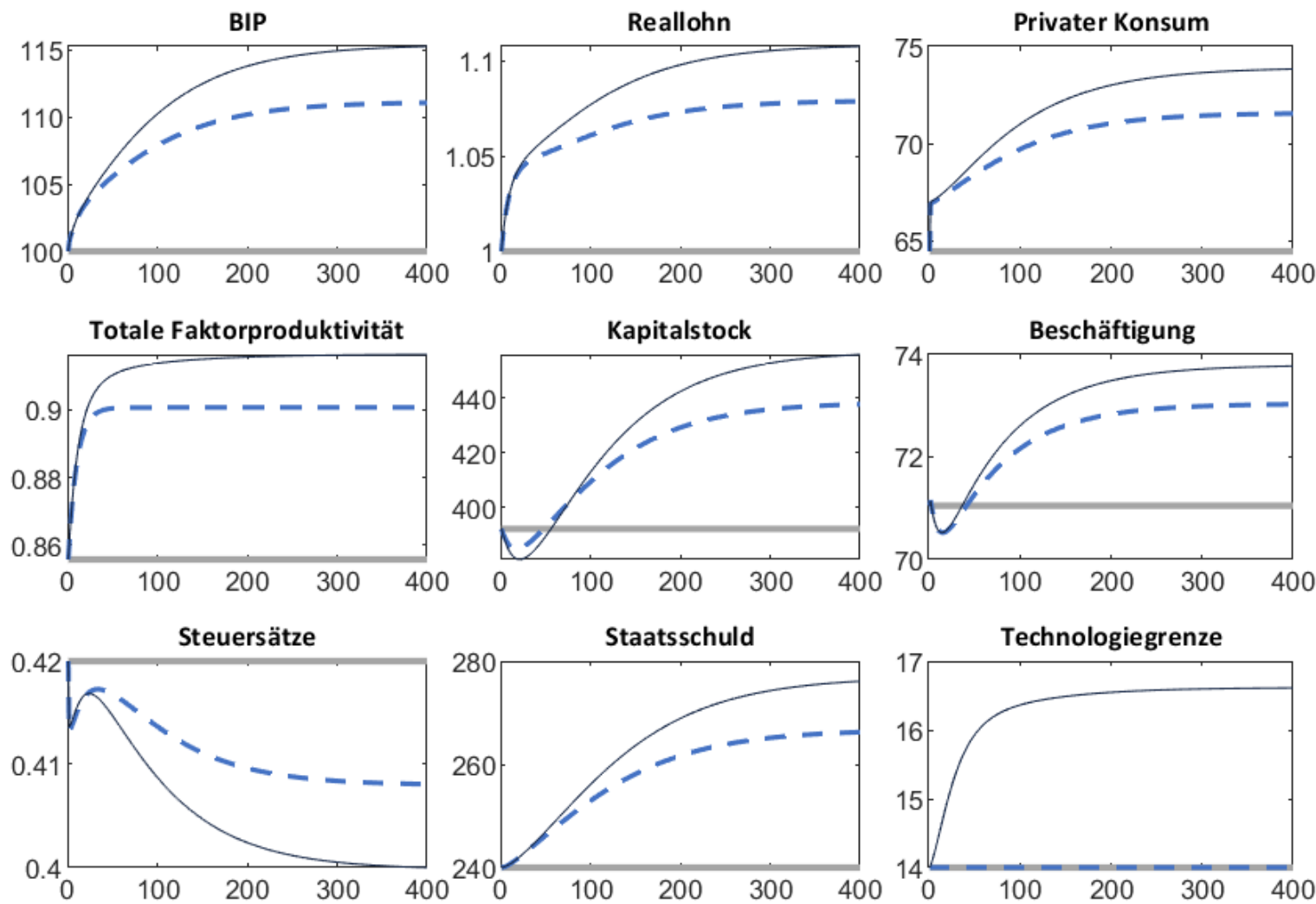

- Basisfall Wachstum, F\&E aktiv $-\quad-$ Wachstum, F\&E passiv

Berechnung und Darstellung: WPZ.

Anmerkung: Wachstumsszenario mit und ohne F\&E-Reaktionen. 


\section{Wirkungen von Forschungsausgaben auf Mikroebene}

Die empirische Forschung zeigt überwiegend positive Effekte der F\&E-Förderung auf verschiedene Aspekte des Unternehmenserfolgs. So kommen z.B. Czarnitzkia und Hussinger (2018) zum Ergebnis, dass öffentlich finanzierte F\&E das Patentaufkommen deutscher Unternehmen erhöht und keine wesentlichen Mitnahmeeffekte zur Folge haben, die lediglich privat finanzierte durch öffentlich finanzierte Forschung ersetzt. Farre-Mensa et al. (2016) quantifizieren den kausalen Effekt einer Patentgewährung auf den nachfolgenden Erfolg US-amerikanischer Unternehmen und stellen positive Auswirkungen auf Beschäftigung und Umsatz fest. Im Vergleich zu den Unternehmen, deren erstes Patentgesuch abgelehnt wurde, haben Unternehmen, deren Patent genehmigt wird, drei Jahre nach der Patentgewährung eine um 20 Prozentpunkte höhere Beschäftigung und einen um 22 Prozentpunkte höheren Umsatz. Nach fünf Jahren wächst der Unterschied zwischen den Unternehmen noch weiter und beträgt schließlich 36 Prozentpunkte bei der Beschäftigung und 51 Prozentpunkte beim Umsatz.

Die empirische Forschung liefert jedoch keine punktgenauen Ergebnisse. Die Schätzungen zu den Auswirkungen auf den Unternehmenserfolg unterscheiden sich bisweilen recht erheblich. Ein Aspekt davon sind die Unterschiede in den Wirkungen nach Branchen. Ortega-Argilés et al. (2010) vergleichen den Einfluss von F\&E auf die Arbeitsproduktivität nach Technologieklassen des verarbeitenden Gewerbes und zeigen für mehrere EU-Mitgliedstaaten, dass F\&E insbesondere in Hochtechnologie-Branchen die Arbeitsproduktivität steigert. Typischerweise verhalten sich F\&EAusgaben eher prozyklisch, d.h. sie steigen im Boom überdurchschnittlich stark an, und fallen in der Rezession umso stärker zurück. So zeigen auch Aristei et al. (2017) für die EU-Mitgliedstaaten, dass die F\&E-Förderung den Rückgang der privaten F\&E-Investitionen in der letzten Krise mildern konnte.

Die folgende Analyse soll für Österreich zeigen, wie sich Förderungen der FFG auf Aspekte des Unternehmenserfolgs wie Beschäftigung, Umsatz und Überlebensquote auswirken. Diese Effekte haben sowohl einzelwirtschaftliche als auch gesamtwirtschaftliche Auswirkungen. Wenn nach einer Förderung die F\&E-Quote steigt, das Unternehmenswachstum beschleunigt und die Überlebenschancen hebt, dann werden die Unternehmen profitabler und größer. Ihr Marktwert steigt. Das ist der einzelwirtschaftliche Ertrag der F\&E. Innovative Unternehmen sind jedoch auch eine Quelle von Wissens-Spill-Overs für andere Unternehmen und fördern deren Wachstum. In der Jagd um Marktanteile bedeutet jedoch der Erfolg der innovativen Unternehmen auch mehr Konkurrenz für die anderen. Lucking et al. (2018) zeigen für die USA, dass die Spill-Over-Effekte den Marktwert der davon profitierenden Unternehmen signifikant steigern. Das bedeutet, dass die positiven Effekte der Technologie-Spill-Over die negativen Effekte erhöhter Konkurrenz übertreffen. Die Ergebnisse ähneln jenen von Bloom et al. (2013), die ebenfalls einen positiven Nettoeffekt von F\&E-Spill-Over-Effekten festgestellt haben.

Lucking et al. (2018) berechnen und vergleichen die privaten und sozialen Renditen von F\&E. Die private Rendite folgt aus der gesteigerten Wertschöpfung eines Unternehmens aufgrund der eigenen F\&E-Aktivitäten. Die positiven externen Effekte multiplizieren jedoch den gesamtwirtschaftlichen Ertrag der privaten F\&E. Innovative Unternehmen lösen einen Multiplikatoreffekt aus und tragen damit mehr als die Summe ihrer Wertschöpfung zum gesamtwirtschaftlichen Wachstum bei. Die soziale Rendite misst die Veränderung der gesamtwirtschaftlichen Wertschöpfung und berücksichtigt damit sowohl positive als auch negative Spill-Over-Effekte. Die Forscherinnen und Forscher stellen fest, dass die private Rendite von F\&E in den USA 13,6 \% beträgt, die soziale Rendite ist dagegen mit $57,7 \%$ rund viermal so hoch. Wer in der Investitionsrechnung alle Kosten veranschlagen muss, aber nur einen Teil der tatsächlichen Erträge berücksichtigt, investiert zu wenig. Weil die soziale Rendite die private übersteigt, könnte die Gesellschaft viel gewinnen, wenn sie eine Ausdehnung der privaten F\&E bewirken könnte. 


\subsection{Datenbasis}

Wir wollen nun mit ökonometrischen Methoden für Österreich die einzelwirtschaftlichen Wirkungen der Forschungsförderung festmachen. Dazu vergleichen wir zunächst, wie sehr sich FFG-geförderte Unternehmen von anderen Unternehmen unterscheiden. Zuerst dokumentieren wir mit MatchingMethoden die statistischen Effekte einer FFG-Förderung auf Wachstum und Beschäftigung der geförderten Unternehmen. Anschließend ermitteln wir mittels Regressionsanalysen, welche Leistungsindikatoren der Unternehmen eine FFG-Förderung beeinflusst.

Für die ökonometrische Matching-Analyse werden drei Datensätze miteinander verbunden:

- „Aurelia“-Datenbank des Bureau van Dijk mit Stand von Oktober 2017: Sie enthält rund 440.000 Unternehmen mit Standort in Österreich sowie eine Reihe spezifischer Variablen. Dazu zählen Umsatz, Börsennotierung, Gründungs- bzw. Umgründungsdatum, Eigenkapital, Beschäftigtenzahl, Branchenzugehörigkeit (nach vierstelliger Untergliederung), Import- und Exporttätigkeit, sowie detaillierte Angaben zum Management (Geschlecht, Wohnsitz), zu den Gesellschaftern (darunter die Herkunft) sowie zum Kapital (darunter die Kategorien Wagniskapital und Private Equity).

- Eine weitere Aurelia-Datenbank mit Stand von Oktober 2020: Die Unternehmens-ID der Datenbank von 2017 wird verknüpft mit neuen Daten zum Umsatz und zur Anzahl der Mitarbeiterinnen und Mitarbeiter. Damit können wir das jeweilige Wachstum 2016-2019 (d.h. bezogen auf das jeweils aktuellste Jahr im Original-Datensatz) berechnen.

- FFG-Daten mit Angaben zu den von der FFG geförderten Unternehmen: Wir suchen in den beiden Aurelia-Datenbanken nach Unternehmen aus der FFG-Datenbank. Stimmen sowohl Unternehmensname als auch Postleitzahl überein, wird das jeweilige Jahr der Förderung (2010-2020) eingetragen.

Wir ergänzen die Angaben in der Aurelia-Datenbank mit zusätzlichen Daten zur branchenspezifischen F\&E-Quote, berechnet nach Daten der Statistik Austria, sowie bezüglich einer etwaigen Risikokapitalfinanzierung (Wagniskapital und/oder Private Equity). Letztere stammen aus eigenen Recherchen (für Details siehe Keuschnigg und Sardadvar, 2019).

Der Datensatz 2017 enthält alle Unternehmen aus der Aurelia-Datenbank mit vollständigen Daten, das sind 55.566 Beobachtungen. Der Datensatz 2020 enthält alle Unternehmen aus der neueren Aurelia-Datenbank mit vollständigen Daten, das sind 64.029 Beobachtungen. Zur Überprüfung des Wachstums werden alle Unternehmen aus dem Datensatz 2017 entfernt, deren ID sich nicht im Datensatz 2020 findet. Übrig bleiben 48.238 Unternehmen. Alle folgenden Berechnungen verwenden diesen reduzierten Datensatz. Von diesen 48.238 Unternehmen haben 3.296 im Zeitraum 2010-2020 zumindest einmal eine FFG-Förderung erhalten, das sind 6,83 \% aller Unternehmen im Sample.

Im Folgenden sind vor allem die Entwicklung der Beschäftigtenzahlen und des Umsatzes von Interesse. Um diese zuverlässig zu messen, darf der Datensatz nur jene Unternehmen enthalten, für die sowohl für das Jahr 2017 als auch für 2020 jeweils aktuelle Beschäftigten- und Umsatzzahlen zur Verfügung stehen. Das sind 38.049 Unternehmen, von welchen 2.260 im Zeitraum 2010-2016 zumindest einmal eine Förderung durch die FFG erhalten haben. Das entspricht einer Quote von $5,94 \%$ aller Unternehmen im Sample.

Um zu berechnen, wie sich Unternehmen mit einer FFG-Förderung im Vergleich zu solchen ohne Förderung entwickelt haben, ist eine Vergleichsgruppe (Kontrollgruppe) von ähnlichen, aber nicht geförderten Unternehmen notwendig. Hierzu sucht man im Sample nach Unternehmen, die keine FFG-Förderung erhalten haben, aber in den anderen Eigenschaften ähnlich waren und daher ähnlich gut für eine Förderung geeignet gewesen wären. Ein Verfahren, um solche Unternehmen zu identifizieren, ist das Propensity-Score-Matching (PSM). Die Methode vergleicht die Unternehmen 
nach ihren quantifizierbaren Eigenschaften. Dazu bilden wir eine "Referenzgruppe" von Merkmalsträgern, die eine "besondere Behandlung" in Form einer FFG-Förderung ("treated“) erfahren haben. Danach identifizieren wir eine möglichst gut vergleichbare Gruppe („Kontrollgruppe“) von Unternehmen, die keine solche „Behandlung“ erfahren haben, d.h. keine FFGFörderung erhalten haben. Wichtig ist, dass sich die Unternehmen der Kontrollgruppe nur in einem Merkmal unterscheidet (FFG-Förderung 2010-2016 erhalten), aber sonst ganz ähnliche Eigenschaften aufweisen und daher genauso gut für eine Förderung in Frage gekommen wären. Um solche Unternehmen zu identifizieren, setzen wir das Verfahren der „nächsten Nachbarn“ („,nearest neighbour ") ein: für jedes tatsächlich FFG-geförderte Unternehmen wird aus dem gesamten Sample jenes Unternehmen herausgepickt, das dem ersten am ähnlichsten ist, aber im Zeitraum 2010-2016 keine FFG-Förderung erhalten hat. Diese ausgewählten Unternehmen bilden die Kontrollgruppe.

Tabelle 4 zeigt die Ergebnisse des PSM-Verfahrens. Die Tabelle vergleicht die arithmetischen Mittelwerte der Variablen für drei Gruppen von Unternehmen. Die „Basisgruppe“ ist die mit Abstand größte Gruppe, da sie ohne Einschränkungen alle 38.048 Unternehmen des Samples aus 2017 mit vollständigen Daten enthält. Die „Referenzgruppe“ enthält nur jene Firmen im Sample, die 2010-2016 irgendeine FFG-Förderung erhalten haben. Angesichts der selektiven Fördervergabe ist sie mit 2.260 Unternehmen nur ein kleiner Teil des gesamten Samples. Die „Kontrollgruppe“ ist genau gleich groß, denn sie enthält im Sample von 2017 für jedes geförderte Unternehmen genau ein anderes, dass in den Eigenschaften dem geförderten am ähnlichsten ist („nearest neighbor“). Die Tabelle zeigt daher, dass die Eigenschaften der Kontroll- und Referenzgruppen für das Jahr 2017 per Konstruktion zwar „möglichst ähnlich“, aber nicht exakt identisch sind. Die Ähnlichkeit ist eben statistisch nur annäherungsweise erreichbar.

Tabelle 4: Arithmetische Mittelwerte der Basis-, Referenz- und der Kontrollgruppen nach Propensity-Score Matching, 2017

\begin{tabular}{|l|l|l|l|}
\hline & Basis & Referenz & Kontroll \\
\hline Beschäftigte (Tsd.) & 38,92 & 121,76 & 98,55 \\
\hline Umsatz (Tsd. €) & 16325,57 & 41536,62 & 38488,19 \\
\hline Börsennotierung (=1, wenn ja) & $0,13 \%$ & $0,44 \%$ & $0,27 \%$ \\
\hline Alter seit Gründung & 25,47 & 26,92 & 26,73 \\
\hline Alter seit Umgründung & 18,50 & 19,60 & 19,96 \\
\hline Eigenkapital (Tsd. €) & 905518,43 & 2199199,37 & 1616254,14 \\
\hline Umsatz je Mitarbeiter/-in (Tsd. €) & 699,48 & 973,71 & 1277,94 \\
\hline Unternehmen in Konzerngruppe & 28,67 & 45,32 & 41,44 \\
\hline Anzahl Gesellschafter & 1,96 & 2,29 & 2,25 \\
\hline Anzahl Tochtergesellschaften & 0,70 & 1,87 & 1,41 \\
\hline Anzahl Geschäftsführer/-innen & 1,53 & 1,91 & 1,87 \\
\hline Anteil österr. Geschäftsführer/-innen & $95,66 \%$ & $95,72 \%$ & $95,18 \%$ \\
\hline Anteil dt. Geschäftsführer/-innen & $2,58 \%$ & $2,78 \%$ & $2,67 \%$ \\
\hline Anteil schweiz. Geschäftsführer/-innen & $0,45 \%$ & $0,52 \%$ & $0,84 \%$ \\
\hline Anteil and. Geschäftsführer/-innen & $1,39 \%$ & $1,31 \%$ & $1,54 \%$ \\
\hline Importquote (in \%) & $10,90 \%$ & $15,44 \%$ & $15,72 \%$ \\
\hline Exportquote (in \%) & $5,99 \%$ & $20,79 \%$ & $21,06 \%$ \\
\hline ausl. Gesellschafter (=1, wenn ja) & $11,40 \%$ & $16,86 \%$ & $17,74 \%$ \\
\hline F\&E-Quote & $2,79 \%$ & $7,07 \%$ & $6,76 \%$ \\
\hline Branche A & $0,51 \%$ & $0,31 \%$ & $0,49 \%$ \\
\hline Branche B & $0,33 \%$ & $0,75 \%$ & $0,88 \%$ \\
\hline
\end{tabular}




\begin{tabular}{|c|c|c|c|}
\hline & Basis & Referenz & Kontroll \\
\hline Branche C & $15,51 \%$ & $38,36 \%$ & $37,48 \%$ \\
\hline Branche D & $0,58 \%$ & $1,90 \%$ & $2,08 \%$ \\
\hline Branche E & $0,77 \%$ & $1,15 \%$ & $1,28 \%$ \\
\hline Branche F & $17,67 \%$ & $7,70 \%$ & $6,86 \%$ \\
\hline Branche G & $27,87 \%$ & $15,75 \%$ & $16,33 \%$ \\
\hline Branche $\mathrm{H}$ & $5,01 \%$ & $1,95 \%$ & $2,30 \%$ \\
\hline Branche I & $5,08 \%$ & $0,31 \%$ & $0,31 \%$ \\
\hline Branche J & $5,51 \%$ & $13,14 \%$ & $14,29 \%$ \\
\hline Branche K & $2,20 \%$ & $1,19 \%$ & $1,33 \%$ \\
\hline Branche L & $4,11 \%$ & $1,15 \%$ & $0,62 \%$ \\
\hline Branche M & $10,40 \%$ & $14,34 \%$ & $13,72 \%$ \\
\hline Branche N & $4,43 \%$ & $1,99 \%$ & $2,04 \%$ \\
\hline Wien & $20,96 \%$ & $14,51 \%$ & $14,82 \%$ \\
\hline Niederösterreich & $15,67 \%$ & $15,80 \%$ & $15,18 \%$ \\
\hline Burgenland & $2,38 \%$ & $2,17 \%$ & $2,12 \%$ \\
\hline Steiermark & $12,37 \%$ & $17,12 \%$ & $17,70 \%$ \\
\hline Kärnten & $6,07 \%$ & $6,28 \%$ & $6,15 \%$ \\
\hline Tirol & $10,28 \%$ & $8,23 \%$ & $7,83 \%$ \\
\hline Oberösterreich & $18,49 \%$ & $24,47 \%$ & $25,04 \%$ \\
\hline Salzburg & $8,24 \%$ & $5,97 \%$ & $5,88 \%$ \\
\hline Vorarlberg & $5,46 \%$ & $5,40 \%$ & $5,22 \%$ \\
\hline Risikokapital & $0,28 \%$ & $1,42 \%$ & $0,84 \%$ \\
\hline$n$ & 38.048 & 2.260 & 2.260 \\
\hline
\end{tabular}

Daten wie im Text dokumentiert; Berechnung: WPZ Research.

Anmerkungen: $n$ bezeichnet die Anzahl der Beobachtungen; Branchenbezeichnungen: A: Land- und Forstwirtschaft; Fischerei, B: Bergbau und Gewinnung von Steinen und Erden; C: Herstellung von Waren; D: Energieversorgung; E: Wasserversorgung; Abwasser- und Abfallentsorgung und Beseitigung von Umweltverschmutzungen; F: Bau; G: Handel; Instandhaltung und Reparatur von Kraftfahrzeugen; H: Verkehr und Lagerei; I: Beherbergung und Gastronomie; J: Information und Kommunikation; K: Erbringung von Finanz- und Versicherungsdienstleistungen; L: Grundstücks- und Wohnungswesen; M: Erbringung von freiberufl., wissenschaftl. und techn. Dienstleistungen; N: Erbringung von sonstigen wirtschaftlichen Dienstleistungen

Wenig überraschend ist in Tabelle 4 die wesentlich höhere F\&E-Quote der FFG-geförderten Unternehmen im Vergleich zur Basisgruppe. Das erklärte Kriterium für die Vergabe einer Förderung ist das innovative Potential der Projektanträge. Unternehmen, die bereits sehr forschungsintensiv sind, sind daher im Wettbewerb um Fördermittel erfolgreicher. Aus einem ähnlichen Grund erhalten FFG-geförderte Unternehmen wesentlich häufiger eine Finanzierung mit Risikokapital (Wagniskapital oder Private Equity).

In Tabelle 5 werden einige Werte der Basis-, Referenz- und Kontrollgruppen für die Folgejahre angegeben. Es handelt sich um dieselben Gruppen von Unternehmen wie in Tabelle 4, aber die Werte können nun - abhängig von der Entwicklung in den Jahren nach dem Jahr, für das das PropensityScore-Matching durchgeführt wurde - abweichen und geben einen ersten Aufschluss über die Entwicklung von FFG-geförderten Unternehmen im Vergleich zu ansonsten ähnlichen, aber nicht geförderten Unternehmen. Es fällt auf, dass die Referenzgruppe nun bei Mitarbeiterzahl und Umsatz wesentlich höhere Werte aufweist als die Kontrollgruppe, die Unternehmen der Referenzgruppe sind somit schneller gewachsen. 
Tabelle 5: Arithmetische Mittelwerte der Basis-, Referenz- und der Kontrollgruppen in den Folgejahren

\begin{tabular}{|l|l|l|l|}
\hline & Basis & Referenz & Kontroll \\
\hline Beschäftigte 2020 (Tsd.) & 40,63 & 130,45 & 98,95 \\
\hline Umsatz 2020 (Tsd. €) & 14094,22 & 46037,54 & 39446,24 \\
\hline Umsatz je Mitarbeiter/-in 2020 (Tsd. €) & 689,88 & 640,48 & 1105,93 \\
\hline FFG 2017 & $2,19 \%$ & $27,65 \%$ & $1,50 \%$ \\
\hline FFG 2018 & $2,21 \%$ & $25,75 \%$ & $1,55 \%$ \\
\hline FFG 2019 & $1,85 \%$ & $21,73 \%$ & $1,33 \%$ \\
\hline FFG 2020 & $1,45 \%$ & $17,61 \%$ & $0,97 \%$ \\
\hline$n$ & 38.048 & 2.260 & 2.260 \\
\hline
\end{tabular}

Daten wie im Text dokumentiert; Berechnung: WPZ Research.

Anmerkungen: $n$ bezeichnet die Anzahl der Beobachtungen; Branchenbezeichnungen: A: Land- und Forstwirtschaft; Fischerei, B: Bergbau und Gewinnung von Steinen und Erden; C: Herstellung von Waren; D: Energieversorgung; E: Wasserversorgung; Abwasser- und Abfallentsorgung und Beseitigung von Umweltverschmutzungen; F: Bau; G: Handel; Instandhaltung und Reparatur von Kraftfahrzeugen; H: Verkehr und Lagerei; I: Beherbergung und Gastronomie; J: Information und Kommunikation; K: Erbringung von Finanzund Versicherungsdienstleistungen; L: Grundstücks- und Wohnungswesen; M: Erbringung von freiberufl., wissenschaftl. und techn. Dienstleistungen; N: Erbringung von sonstigen wirtschaftlichen Dienstleistungen

Zusätzlich fällt in Tabelle 5 auf, dass Unternehmen, die 2010-2016 eine FFG-Förderung erhalten haben, auch in den Folgejahren häufiger auf Fördermitteln zählen konnten. Die Quoten liegen bei $27,65 \%$ im Jahr 2017, 25,75 \% in 2018, 21,73 \% in 2019 und 17,61 \% in 2020. Interessant ist die Diskrepanz zur Kontrollgruppe: Obwohl die Unternehmen ähnlich zur Referenzgruppe sind, hat nur ein Bruchteil der Referenzgruppe in den Folgejahren eine FFG-Förderung erhalten. Nachdem eine FFG-Förderung zusätzliche Mittel für F\&E bereitstellt und außerdem die F\&E-Investitionen anregt, steigt mit der Förderung die F\&E-Intensität gleich zweifach. Es ist zu erwarten, dass zunehmende F\&E-Aktivitäten sich im innovativen Potential der Projektanträge niederschlagen und die Erfolgswahrscheinlichkeit bei der Mittelvergabe steigern. Zusätzlich dürften diese Unternehmen über mehr Erfahrung mit Anträgen verfügen.

\subsection{FFG Förderung und Unternehmensentwicklung}

Mit F\&E verbessern die Unternehmen die Wettbewerbsfähigkeit und bauen ihre Marktanteile aus. Aber wie stark ist der Effekt? Wie sehr können Forschungsförderung und private F\&E die Unternehmensentwicklung beeinflussen? Nach der Matching-Methode sind die Unternehmen der Referenzgruppe, die eine FFG-Förderung in den Jahren 2010-2016 erhalten haben, ganz ähnlich zu den nicht-geförderten und Unternehmen weniger F\&E-intensiver Branchen der Kontrollgruppe. Sie starten daher im Jahr 2017 in einer ähnlichen Ausgangsposition. Danach läuft die Entwicklung auseinander. Abbildung 16 vergleicht die Wachstumsraten der Beschäftigtenzahl und des Umsatzes über den gesamten Zeitraum 2016 bis 2019 und zeigt beeindruckende Unterschiede. Die geförderten Unternehmen übertreffen die anderen sehr deutlich: Das Wachstum der Zahl der Mitarbeiterinnen und Mitarbeiter liegt bei 7,14 \%, in der Kontrollgruppe lediglich bei 0,40 \%. Das Umsatzwachstum (nicht inflationsbereinigt) liegt bei 10,84\%, in der Kontrollgruppe dagegen bei nur 2,49\%. 
Abbildung 16: Wachstum von Beschäftigung und Umsatz, 2016-2019

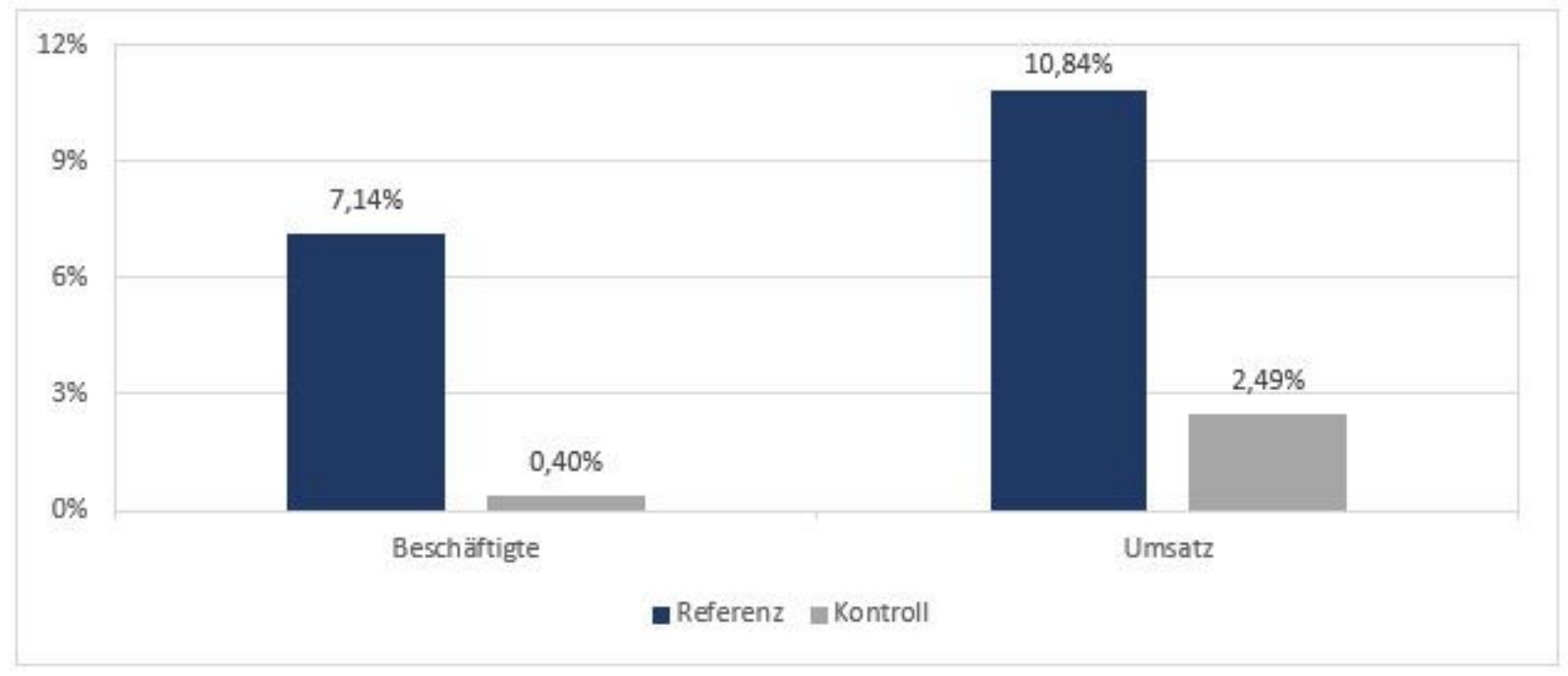

Quelle: Arithmetische Mittelwerte. Daten wie im Text dokumentiert; Berechnung und Darstellung: WPZ Research.

F\&E-Anreize können in verschiedenen Branchen sehr unterschiedliche Wirkungen haben. Um diese Unterschiede festzumachen, berechnet Abbildung 17 die Wachstumsraten der Beschäftigtenzahlen separat für ausgewählte Branchen, getrennt nach Referenz- und der Kontrollgruppe. Dabei beschränken wir uns auf Branchen mit zumindest 100 Beobachtungen. ${ }^{12}$ Wie Tabelle 4 zeigt, sind einige Branchen bereits in der Basisgruppe nur mit sehr wenigen Unternehmen vertreten. Zwangsläufig werden die Referenz- und Kontrollgruppe jeweils sehr klein und verlieren an Aussagekraft. Das gilt z.B. für die Branchen A (Landwirtschaft) und B (Bergbau), die nur sieben bzw. 16 Unternehmen in den Referenzgruppen zählen. Wesentlich aussagekräftiger sind die Ergebnisse für Branche C (Herstellung von Waren bzw. verarbeitendes Gewerbe), für die eine Tabelle analog zu Tabelle 4 erstellt wurde, sie enthält nur die Unternehmen des verarbeitenden Gewerbes und findet sich im Anhang. Auffällig ist die hohe Anzahl ausländischer Gesellschafter sowie Geschäftsführerinnen und Geschäftsführern im Vergleich zur Basisgruppe, nicht jedoch im Vergleich zur Kontrollgruppe. Die hohen Werte hier können mit der Größe der Unternehmen zusammenhängen, da diese tendenziell auch internationaler ausgerichtet sind.

Auch im verarbeitenden Gewerbe wächst die Beschäftigung in der Referenzgruppe wesentlich schneller als in der Kontrollgruppe, wie Abbildung 17 zeigt. Konkret nimmt die Beschäftigung der durch die FFG geförderten Unternehmen um 4,95\% zu. Im Vergleich dazu fallen die nicht-geförderten Unternehmen mit einem Beschäftigungswachstum von nur 1,73\% stark ab. Allerdings sind auch andere Einflussfaktoren für das Unternehmenswachstum wichtig, so dass der Unterschied nicht ursächlich und ausschließlich mit der FFG-Förderung erklärt werden kann. Zudem ist auch das Volumen der Förderungen fallweise recht klein. Die Ergebnisse deuten aber zumindest darauf hin, dass die FFG solche Unternehmen des verarbeitenden Gewerbes auswählt, die über ein hohes Wachstumspotenzial verfügen. Es ist eine volkswirtschaftlich wichtige Funktion, die Fördermittel dorthin zu lenken, wo das Potential am größten ist.

12 Die Branche mit der kleinsten Zahl an Beobachtungen in Abbildung 17 ist Branche F (Bau) mit 174 Beobachtungen (vgl. Tabelle 4: Die Multiplikation des Branchenanteils mit der Samplegröße ergibt die Anzahl der Unternehmen der jeweiligen Branche). 
Abbildung 17: Wachstum der Beschäftigten nach Branchen, 2016-2019

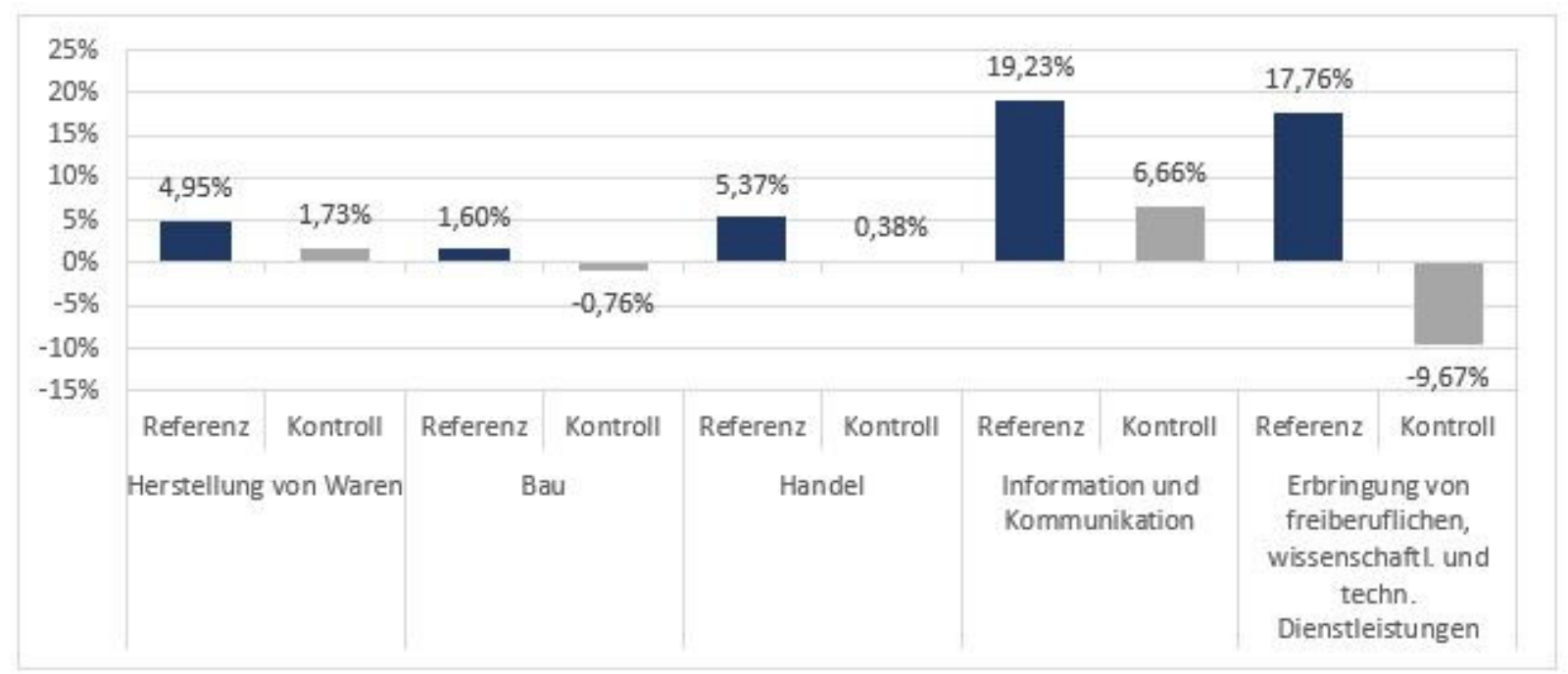

Quelle: Arithmetische Mittelwerte. Daten wie im Text dokumentiert; Berechnung und Darstellung: WPZ Research.

Die ausgewählten Unternehmen erscheinen sehr forschungsintensiv. Sowohl die branchenspezifische F\&E-Quote als auch der Anteil risikofinanzierter Unternehmen sind in der Referenzgruppe der geförderten Unternehmen höher als in den anderen beiden Gruppen. Der Anteil der Unternehmen mit Standort Wien ist hingegen auffallend niedrig, obwohl sich Humankapital (Sardadvar, 2019) sowie risikofinanzierte Unternehmen im Raum Wien konzentrieren (Keuschnigg und Sardadvar, 2019; Sardadvar, 2020).

Weniger aussagekräftig sind die Wachstumszahlen für die Versorger-Branchen D (Energie) und E (Wasser) mit nur 43 bzw. 26 Unternehmen in der Referenzgruppe. Einige wenige recht große Unternehmen haben Mitarbeiterinnen und Mitarbeiter abgebaut, was sich in den Wachstumszahlen nach dem arithmetischen Mittel deutlich bemerkbar macht. Weitere Branchen mit geringen Fallzahlen und daher wenig Aussagekraft sind H (Verkehr, 44 Unternehmen in der Referenzgruppe), I (Beherbergung und Gastronomie, 7 Unternehmen), K (Finanz und Versicherung, 27 Unternehmen), L (Grundstücks- und Wohnungswesen, 26 Unternehmen) und $\mathrm{N}$ (sonstige wirtschaftliche Dienstleistungen, 45 Unternehmen).

Im Unterschied zu diesen Branchen mit wenigen Beobachtungen lassen die Branchen F (Bau, 174 Unternehmen), G (Handel, 356 Unternehmen), J (Information und Kommunikation, 297 Unternehmen) und M (freiberufliche, wissenschaftliche und technische Dienstleistungen, 324 Unternehmen) statistisch aussagekräftige Ergebnisse zu. In diesen vier Branchen ist das Wachstum der Beschäftigtenzahl deutlich höher als in der Kontrollgruppe. Gerade die Branchen „Information und Kommunikation" und „freiberufliche, wissenschaftliche und technische Dienstleistungen" stehen für eine hohe Innovations- und Forschungsintensität. Die F\&E-Quoten sind im Vergleich zur Basisgruppe höher (6,48 \% zu 5,99 \% sowie 20,14 \% zu 11,97 \%). In diesen F\&E-intensiven Branchen fällt der Unterschied im Beschäftigtenwachstum zwischen geförderten und nicht geförderten, aber sonst ähnlichen Unternehmen mit 19,23 \% zu 6,66 \% in Information und Kommunikation und mit $17,76 \%$ zu $-9.67 \%$ in wissenschaftsnahen Dienstleistungen besonders groß aus.

Abbildung 18 stellt für dieselben Branchen das Umsatzwachstum dar. Die Umsätze der geförderten Unternehmen (Referenzgruppe) nehmen von 2016 bis 2019 durchwegs stärker zu als jene von anderen, aber sonst ähnlichen Unternehmen (Kontrollgruppe). Das kommt sowohl in der Branche „Herstellung von Waren“ zum Ausdruck (15,94 \% zu 8,71\%) wie auch in den forschungsintensiven 
Branchen „Information und Kommunikation“ (32,50\% $\mathrm{zu} \quad 8,27 \%)$ und „freiberufliche, wissenschaftliche und technische Dienstleistungen“ (-20,22\% zu -62,74\%). ${ }^{13}$ In den weit weniger forschungsintensiven Branchen „Bau“ und „Handel“ übertreffen die FFG-geförderten Unternehmen das Umsatzwachstum der Kontrollgruppe sogar bei weitem, mit 17,18 \% zu 2,91 \% bzw. 17,67 \% zu 2,17 \%. Demnach konzentriert sich die FFG-Förderung im Jahr 2016 und davor auf Unternehmen mit hohem Potential, die in den Folgejahren ihren Umsatz erheblich steigern konnten. Allerdings gibt es auch an dieser Stelle zu bedenken, dass das Umsatzwachstum auch von anderen Einflussgrößen abhängt, so dass die Unterschiede nicht allein und ausschließlich auf die FFG-Förderung zurückzuführen sind.

\section{Abbildung 18: Wachstumsraten der Umsätze nach Branchen, 2016-2019}

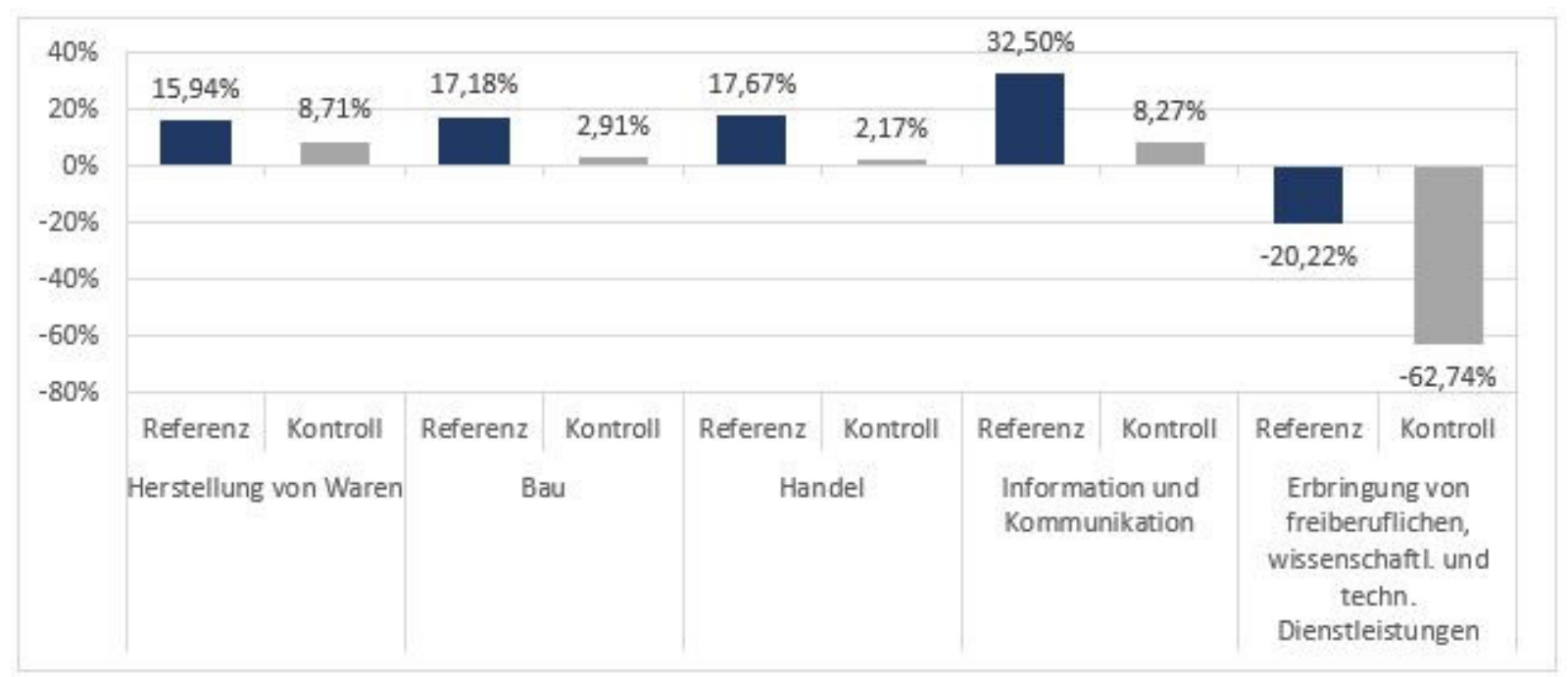

Quelle: Arithmetische Mittelwerte. Daten wie im Text dokumentiert; Berechnung und Darstellung: WPZ Research.

Unabhängig von anderen potentiellen Einflussfaktoren kann man zusammenfassend mit einiger Sicherheit sagen, dass Unternehmen, die 2010-2016 durch die FFG gefördert wurden, hinsichtlich Beschäftigung und Umsatz 2016-2019 schneller gewachsen sind als ansonsten vergleichbare Unternehmen, die keine FFG-Förderung erhalten haben. Die Auswahl durch die FFG und/oder die Entscheidung eines Unternehmens, einen entsprechenden Antrag einzureichen, hängen also mit dem anschließenden Unternehmenswachstum positiv zusammen.

Die bisherigen Ergebnisse beziehen sich auf Unternehmen, die in der Zeitspanne 2017 bis 2020 überlebt haben und damit zu beiden Zeitpunkten im Datensatz vorhanden sind. Eine andere Frage ist, ob sich FFG-geförderte Unternehmen hinsichtlich ihrer Überlebenschance von anderen ähnlichen Unternehmen unterscheiden. Wir lassen nun alle 55.566 Unternehmen im Datensatz 2017, einschließlich solcher, die im Jahr 2020 nicht mehr existierten, in die Analyse eingehen und suchen mit dem PSM-Verfahren für jedes der FFG-geförderten Unternehmen (Referenzgruppe) jeweils das nicht-geförderte Unternehmen, dass dem geförderten in allen anderen Eigenschaften am meisten ähnelt (Kontrollgruppe). In beiden Gruppen kommt es nach 2017 zu Austritten, aber eben unterschiedlich häufig.

13 Das negative Wachstum in Branche M (Erbringung von freiberuflichen, wissenschaftlichen und technischen Dienstleistungen) ist auf Ausreißer zurückzuführen. Beim mittleren Umsatzwachstum nach dem Median ergeben sich durchgehend positive Wachstumszahlen (7,50 \% für die Basis-, 30,34 \% für die Referenz- und 9,89 \% für die Kontrollgruppe). 
Tabelle 6: Überlebensraten der geförderten und nicht geförderten Unternehmen

\begin{tabular}{|l|l|l|l|}
\hline & Anzahl & überlebt & Quote \\
\hline FFG-gefördert 2010-2016 & 2.806 & 2.548 & $90,81 \%$ \\
\hline Nicht FFG-gefördert 2010-2016 & 2.806 & 2.490 & $88,74 \%$ \\
\hline
\end{tabular}

Quelle: Daten wie im Text dokumentiert; Berechnung und Darstellung: WPZ Research.

Tabelle 6 zeigt die Ergebnisse. Von den 2.806 FFG-geförderten Unternehmen haben 90,81 \% überlebt, von ebenso vielen Unternehmen in der Kontrollgruppe lediglich 88,74\%. Von der FFG geförderte Unternehmen haben eine höhere Überlebenschance. Dieses Ergebnis ist signifikant mit einem $p$-Wert von 0,00017 , das $95 \%$-Konfidenzintervall liegt zwischen $87,50 \%$ und $89,87 \%$ und somit unter den beobachteten $90,81 \%$.

\subsection{Die Charakteristika geförderter Unternehmen}

Was bestimmt den Erfolg der Unternehmen, eine FFG-Förderung zu erhalten, damit sie mehr, größere und anspruchsvollere F\&E Vorhaben finanzieren können? Mittels Regressionsanalyse ermitteln wir, wie die Erfolgsquote bei den FFG-Förderanträgen mit verschiedenen Unternehmenseigenschaften zusammenhängt. Konkret schätzen wir für den Zeitraum 2010-2020 mit einer logistischen Regression (Logit-Verfahren) die Wahrscheinlichkeit, mit der die Unternehmen mit ihren Anträgen für eine FFG-Förderung erfolgreich sind. Als Datensatz verwenden wir das gesamte Sample mit vollständigen Daten für 2020 (64.029 Unternehmen). Für ein einzelnes Unternehmen nimmt die abhängige Variable den Wert eins an, wenn es irgendwann im Zeitraum von 2010 bis 2020 eine FFGFörderung erhalten hat, ansonsten null. Dann ermitteln wir, welche Eigenschaften der Unternehmen (unabhängige Variable) besonders häufig mit einer Förderung einhergehen. Unter sonst gleichen Bedingungen zeigen positive Vorzeichen der geschätzten Koeffizienten eine höhere Wahrscheinlichkeit an, dass diese Eigenschaft mit einer FFG-Förderung einhergeht. Negative Vorzeichen reduzieren die Wahrscheinlichkeit der Förderung. Bei binären Variablen ist ein Vorzeichen immer in Relation zu allen nicht in die Regression aufgenommenen Variablen zu interpretieren. ${ }^{14}$

Tabelle 7 zeigt die Ergebnisse der ersten Regression. Dabei gehen jene Variablen ein, die am meisten zur Erklärung der Erfolgswahrscheinlichkeit beitragen oder von besonderem Interesse sind. Die Ergebnisse sind bemerkenswert aussagekräftig insofern, als recht viele Variablen einen statistisch signifikanten Einfluss haben. Es sei darauf hingewiesen, dass dieser Ansatz zunächst noch nicht die kausalen Zusammenhänge sicher erklären kann, sondern erst einmal feststellt, welche Eigenschaften der Unternehmen typischerweise mit einer FFG-Förderung einhergehen. Kausale Interpretationen sind zunächst nur mit einem gewissen Vorbehalt möglich, da man davon ausgehen muss, dass eine FFG-Förderung die weitere Entwicklung des Unternehmens günstig beeinflusst. Damit würde ein erfolgreicher Antrag die günstigen Eigenschaften erklären, anstatt dass die Eigenschaften den Erfolg erklären würden. Auch wenn aus ökonomischen Gründen die Richtung der Kausalität klar sein könnte, bliebe sie statistisch unklar. Der Vorteil dieser Herangehensweise ist jedoch die große Anzahl an geförderten Unternehmen (6,17 \% im Datensatz).

14 Im Unterschied zu linearen Regressionen sind die Koeffizienten logistischer Regressionen in ihrer Größe nicht direkt, sondern nur in der qualitativen Richtung interpretierbar (Best und Wolf, 2010), weshalb auf Aussagen über prozentuale Effekte verzichtet wird. Bei binären Variablen (Branchen und Bundesländer) ist der Koeffizient relativ zu den nicht in die Regression aufgenommenen zu interpretieren. Ein positiver, statistisch signifikanter Effekt für den Standort X bedeutet hier, dass dieser relativ zu allen anderen Standorten, die nicht als erklärende Variable in der Ergebnisliste aufscheinen, bevorzugt ist, und analog bei negativen Effekten sowie anderen binären Variablen. 
Tabelle 7: Logit-Regression, abhängige Variable: FFG-Förderung 2010-2020

\begin{tabular}{|c|c|c|}
\hline & Koeffizient & Standardfehler \\
\hline Konstante & $-4,5700$ & $0,0804^{* * *}$ \\
\hline Kapital & $<0,0000$ & $0,0000^{*}$ \\
\hline Letzter Umsatz & $<0,0000$ & $0,0000^{* * *}$ \\
\hline F\&E-Quote & 3,9820 & $0,2652 * * *$ \\
\hline Anzahl Gesellschafter & 0,0191 & $0,0042^{* * *}$ \\
\hline Anz. österr. Geschäftsführer/-innen & 0,0634 & $0,0099 * * *$ \\
\hline Exportquote & 0,0133 & $0,0007^{* * *}$ \\
\hline ausl. Gesellschafter (=1, wenn ja) & 0,0807 & 0,0667 \\
\hline Branche $\mathrm{C}$ & 0,8370 & $0,0573^{* * *}$ \\
\hline Branche D & 2,0440 & $0,1496^{* * *}$ \\
\hline Branche E & 1,0030 & $0,1687^{* * *}$ \\
\hline Branche $\mathrm{H}$ & $-0,4801$ & $0,1187^{* * *}$ \\
\hline Branche I & $-1,0600$ & $0,1394^{* * *}$ \\
\hline Branche J & 1,6430 & $0,0612^{* * *}$ \\
\hline Branche M & 0,6150 & $0,0767^{* * *}$ \\
\hline Branche N & $-0,5030$ & $0,1254^{* * *}$ \\
\hline Wien & $-0,4100$ & $0,0503^{* * *}$ \\
\hline Steiermark & 0,2867 & $0,0511^{* * *}$ \\
\hline Salzburg & $-0,2604$ & $0,0740 * * *$ \\
\hline $\log$ (Beschäftigte) & 0,5224 & $0,0163^{* * *}$ \\
\hline $\log$ (Alter) & $-0,1032$ & $0,0228^{* * *}$ \\
\hline $\log ($ Unternehmen in Konzerngruppe) & $-0,0052$ & 0,0135 \\
\hline Risikokapital & 1,2260 & $0,2327^{* * *}$ \\
\hline AIC & 23895,98 & \\
\hline$n$ & 64.029 & \\
\hline
\end{tabular}

Quelle: Daten wie im Text dokumentiert; Berechnung: WPZ Research

Anmerkung: Die Standardfehler geben Auskunft über die statistische Irrtumswahrscheinlichkeit, wobei $*$ eine Wahrscheinlichkeit von $<10 \%$, ** von $<5 \%$ und ${ }^{* * *}$ von $<1 \%$ bezeichnet; „log“ zeigt an, dass die Variable für die Regression logarithmiert wurde; AIC bezeichnet den Wert des Akaike-Informationskriteriums, welches die Modellgüte anzeigt (je niedriger der Wert, desto besser); $n$ die Samplegröße; C: Herstellung von Waren; D: Energieversorgung; E: Wasserversorgung; Abwasser- und Abfallentsorgung und Beseitigung von Umweltverschmutzungen; H: Verkehr und Lagerei; I: Beherbergung und Gastronomie; J: Information und Kommunikation; M: Erbringung von freiberufl., wissenschaftl. und techn. Dienstleistungen; N: Erbringung von sonstigen wirtschaftlichen Dienstleistungen

Die Unternehmensgröße ergibt kein klares Bild. Unternehmen mit mehr Umsatz und höherem Kapitalstock haben keine höhere oder geringere Erfolgswahrscheinlichkeit als andere Unternehmen. Die Anzahl der Gesellschafter und die Anzahl der Beschäftigten erhöhen hingegen die Erfolgswahrscheinlichkeit. Eindeutig positiv korreliert sind die branchenspezifische F\&E-Quote und eine Finanzierung durch Risikokapital. Es erscheint ökonomisch plausibel, dass Unternehmen, die viel in F\&E investieren, Projektanträge mit größerem Innovationspotential einreichen und deshalb erfolgreicher sind. Ähnliches gilt für Risikokapital, welches vorwiegend auf die Finanzierung innovativer Unternehmen spezialisiert ist. Außerdem sind die geförderten Unternehmen eher jung (das Alter hat einen negativen Einfluss) und international ausgerichtet (die Exportquote und ausländische Gesellschafter haben jeweils einen positiven Einfluss). Unternehmen, die auf Auslandsmärkten aktiv sind, sind einem besonders harten Wettbewerb ausgesetzt und müssen daher besonders innovativ sein. In Folge wird man eine höhere Erfolgsquote bei FFG-Anträgen erwarten. 
Daneben zeigt die Schätzung systematische Unterschiede der Erfolgswahrscheinlichkeit nach Branchen und Bundesländern. Die Branchen C (verarbeitendes Gewerbe), D (Energieversorgung), E (Wasserversorgung), J (Information und Kommunikation) und $\mathrm{M}$ (freiberufliche, wissenschaftliche und technische Dienstleistungen) weisen überdurchschnittlich hohe Erfolgsquoten auf. Eine Zugehörigkeit zu den Branchen H (Verkehr), I (Beherbergung und Gastronomie) und N (sonstige wirtschaftliche Dienstleistungen) reduziert hingegen unter sonst gleichen Umständen die Wahrscheinlichkeit einer Förderung durch die FFG.

Auch der Zusammenhang mit dem Standort ist bemerkenswert, da Wien und, etwas schwächer ausgeprägt, Salzburg negative und statistisch hoch signifikante Vorzeichen zeigen, die Steiermark hingegen ein positives. Das ist weder auf die Branchen noch auf die Technologieintensität oder das Vorhandensein von Risikokapital zurückzuführen, da für diese Variablen in der Regression kontrolliert wird. Dafür sind wohl eher andere Einflüsse verantwortlich, für die in der Regression mangels Daten nicht kontrolliert wird. So bietet z.B. Wien selbst sehr viel an Forschungsförderung an. Dieses Angebot könnte teilweise in Konkurrenz zu FFG-Förderungen stehen.

In einem zweiten Schritt weisen wir der abhängigen Variable nur dann den Wert eins zu, wenn ein Unternehmen 2020 (bis zum Zeitpunkt der Übermittlung der Daten durch die FFG an WPZ Research) eine FFG-Förderung erhalten hat. So wird eine kausale Interpretation möglich: Die Unternehmenseigenschaften stammen aus demselben Jahr, so dass sie durch den Erhalt einer Förderung nicht mehr beeinflussbar sind. Andererseits werden die Ergebnisse statistisch in der Regel weniger aussagekräftig, weil nur recht wenige Unternehmen 2020 eine Förderung erhalten haben (1,35\% im Datensatz).

Wir führen die Regression zweimal durch, zuerst mit denselben Variablen wie in Tabelle 7, und dann mit einer zusätzlichen Variablen, die zählt, in wie vielen der vorangegangenen Jahre 2010-2019 das Unternehmen bereits eine FFG-Förderung erhalten hat. Tabelle 8 stellt die Ergebnisse dar, die sehr interessant und aufschlussreich sind. Die Befürchtung, dass die Ergebnisse statistisch weniger aussagekräftig seien, trifft nicht zu. Zwar sind weniger Variable statistisch signifikant, aber die Modellgüte, berechnet nach dem Akaike-Informationskriterium (AIC), ist besser. Vor allem aber bleiben die meisten Variablen statistisch signifikant und verändern ihre Vorzeichen nicht. Die Ergebnisse sind somit robust.

Zunächst gehen wir auf die erste Regression in Tabelle 8 ein. Zu den wenigen Variablen, die im Vergleich zu Tabelle 7 ihre statistische Signifikanz verlieren, zählen der Umsatz und der Kapitalstock. Alle anderen Variablen, die mit der Unternehmensgröße einhergehen, wie die Zahl der Beschäftigten und der Anzahl der Gesellschafter, wirken positiv auf die Erfolgswahrscheinlichkeit. Unter sonst gleichen Umständen steigt die Wahrscheinlichkeit, eine FFG-Förderung zu erhalten, mit der Unternehmensgröße an. Unternehmen in den Branchen C (verarbeitendes Gewerbe) und J (Information und Kommunikation) sowie - etwas weniger ausgeprägt - in Branche M (freiberufliche, wissenschaftliche und technische Dienstleistungen) erhalten häufiger eine Förderung. Dagegen sind die Unternehmen in Branche I (Beherbergung und Gastronomie) seltener erfolgreich.

Die rechte Hälfte der Tabelle wiederholt die Schätzung, wobei jedoch eine zusätzliche erklärende Variable hinzukommt, nämlich die Anzahl der Jahre mit FFG-Förderungen im vorausgehenden Zeitraum von 2010 bis 2019. Das statistische Erklärungsvermögen, angezeigt durch das AICKriterium, verbessert sich wesentlich (je niedriger, desto besser). Zunächst fällt auf, dass der quantitative Einfluss der meisten Variablen abnimmt, weil nun ein Teil der Erfolgswahrscheinlichkeit neu durch die vergangenen Förderungen erklärt wird. 
Tabelle 8: Logit-Regression, abhängige Variable: FFG-Förderung 2020

\begin{tabular}{|c|c|c|c|c|}
\hline & Koeffizient & Standardfehler & Koeffizient & Standardfehler \\
\hline Konstante & $-5,6210$ & $0,1578^{* * *}$ & $-4,8110$ & $0,1767^{* * *}$ \\
\hline Kapital & $<0,0000$ & 0,0000 & $<0,0000$ & 0,0000 \\
\hline Letzter Umsatz & $<0,0000$ & 0,0000 & $<0,0000$ & $0,0000^{* *}$ \\
\hline F\&E-Quote & 5,2290 & $0,5297^{* * *}$ & 2,2610 & $0,5942^{* * *}$ \\
\hline Anzahl Gesellschafter & 0,0107 & $0,0061^{*}$ & 0,0118 & 0,0074 \\
\hline Anz. österr. Geschäftsführer/-innen & 0,0632 & $0,0160^{* * *}$ & 0,0489 & $0,0215^{* *}$ \\
\hline Exportquote & 0,0107 & $0,0013^{* * *}$ & 0,0006 & 0,0016 \\
\hline ausl. Gesellschafter (=1, wenn ja) & 0,1086 & 0,1215 & 0,1792 & 0,1461 \\
\hline Branche C & 0,9435 & $0,1226^{* * *}$ & 0,7664 & $0,1353^{* * *}$ \\
\hline Branche D & 1,5820 & $0,3318^{* * *}$ & 0,6245 & 0,3996 \\
\hline Branche E & 0,3066 & 0,5113 & 0,1168 & 0,5333 \\
\hline Branche $\mathrm{H}$ & $-0,5869$ & $0,3031^{*}$ & $-0,4327$ & 0,3114 \\
\hline Branche I & $-3,2970$ & $1,0020^{* * *}$ & $-3,0790$ & $1,0020^{* * *}$ \\
\hline Branche J & 1,7760 & $0,1228^{* * *}$ & 1,1940 & $0,1383^{* * *}$ \\
\hline Branche M & 0,5875 & $0,1713^{* * *}$ & 0,4544 & $0,1758^{* * *}$ \\
\hline Branche N & $-0,6842$ & $0,3151^{* *}$ & $-0,4346$ & 0,3164 \\
\hline Wien & $-0,4360$ & $0,1044^{* * *}$ & $-0,2591$ & $0,1172^{* *}$ \\
\hline Steiermark & 0,2975 & $0,0990^{* * *}$ & 0,0516 & 0,1186 \\
\hline Salzburg & $-0,3058$ & $0,1575^{*}$ & $-0,1734$ & 0,1780 \\
\hline $\log$ (Beschäftigte) & 0,5438 & $0,0323^{* * *}$ & 0,2732 & $0,0391^{* * *}$ \\
\hline $\log$ (Alter) & $-0,3653$ & $0,0453^{* * *}$ & $-0,0910$ & $0,0328^{* * *}$ \\
\hline $\log$ (Unternehmen in Konzerngruppe) & $-0,0540$ & $0,0266^{* *}$ & $-0,0910$ & $0,0328^{* * *}$ \\
\hline Risikokapital & 0,3418 & 0,3536 & $-1,4400$ & $0,4549^{* * *}$ \\
\hline FFG-Förderungen 2010-2019 & & & 0,6476 & $0,0181^{* * *}$ \\
\hline AIC & 7316,98 & & 5766,887 & \\
\hline$n$ & 64.029 & & 64.029 & \\
\hline
\end{tabular}

Quelle: Daten wie im Text dokumentiert; Berechnung: WPZ Research

Anmerkung: die Standardfehler geben Auskunft über die statistische Irrtumswahrscheinlichkeit, wobei $*$ eine Wahrscheinlichkeit von $<10 \%{ }^{* *}$ von $<5 \%$ und ${ }^{* * *}$ von $<1 \%$ bezeichnet; „log" zeigt an, dass die Variable für die Regression logarithmiert wurde; AIC bezeichnet den Wert des Akaike-Informationskriteriums, welches die Modellgüte anzeigt (je niedriger der Wert, desto besser); $n$ die Samplegröße; Branchenbezeichnungen: C: Herstellung von Waren; D: Energieversorgung; E: Wasserversorgung; Abwasser- und Abfallentsorgung und Beseitigung von Umweltverschmutzungen; H: Verkehr und Lagerei; I: Beherbergung und Gastronomie; J: Information und Kommunikation; M: Erbringung von freiberufl., wissenschaftl. und techn. Dienstleistungen; N: Erbringung von sonstigen wirtschaftlichen Dienstleistungen

Einige Variablen verlieren teilweise an statistischer Signifikanz, da ihr Erklärungsvermögen durch die zusätzliche Variable gewissermaßen aufgesogen wird. Dieser Effekt kommt besonders beim Risikokapital zum Tragen, das nun negativ und statistisch signifikant ist. Es muss also einen Zusammenhang zwischen Risikokapitalfinanzierung und FFG-Förderungen geben. Man kann sich mehrere positive und negative Zusammenhänge vorstellen. Wagnis- und Risikokapital ist vorwiegend auf besonders riskante und innovative Unternehmen spezialisiert, die oft nur schwer Zugang zu anderen Finanzierungsquellen haben. Erfolg in der kompetitiven Fördervergabe wirkt üblicherweise wie ein Qualitätssignal für externe Investoren. Daher gibt es Evidenz, dass mit einer kompetitiven Fördervergabe Wagnisfinanzierung eher möglich wird. Andererseits sind auch Banken und andere Kapitalgeber eher bereit, Finanzierung zu geben, wenn sie angesichts eines hart geprüften und erfolgreichen Förderantrags mit guten Entwicklungschancen des Unternehmens rechnen können. 
Dann wird Risikokapital schlicht weniger gebraucht. Zudem erhält das Unternehmen mit einer Förderung eben auch umfangreiche Finanzmittel und ist daher weniger auf externe Finanzierung angewiesen. Beides zusammen könnte bedeuten, dass eine FFG-Förderung Risikokapital auch ersetzen kann. Was nun genau die Wechselwirkung zwischen FFG-Finanzierung und Risikokapital ist, kann an dieser Stelle nicht abschließend geklärt werden. Tabelle 8 zeigt jedoch, dass eine solche Wechselwirkung ganz offensichtlich besteht.

\subsection{F\&E-Ausgaben, FFG-Förderung und Unternehmenserfolg}

Die Matching-Analyse zeigt bereits, dass FFG-geförderte Unternehmen sich nach Umsatz und Beschäftigung besser entwickeln als andere, sonst vergleichbare Unternehmen. Nun vertiefen wir die Analyse und untersuchen mit anderen ökonometrischen Methoden, ob sich Unternehmen, die mehr Forschung betreiben, nach den Kriterien Wachstum, Beschäftigung, Exporte und Überlebenschancen besser entwickeln. Dazu verknüpfen wir die zusätzlichen Daten, wie sie von der FFG zur Verfügung gestellt wurden, mit den Unternehmen in der Datenbank der WPZ Research (die auf der AureliaDatenbank basiert). Diese zusätzlichen Daten sind der Exportanteil (in \%), der F\&E-Aufwand (in Euro) und die Gesamtförderung durch die FFG (in Euro). Der Vorteil liegt darin, dass nun der F\&EAufwand und das Fördervolumen auf Unternehmensebene vorliegen. Der Nachteil ist, dass sich der Datensatz auf jene Unternehmen reduziert, die eine FFG-Förderung erhalten haben, und noch einmal auf jene, für die im FFG-Datensatz die entsprechenden Daten tatsächlich verfügbar sind. Um einen verzerrenden Einfluss auszuschalten, bereinigen wir das Sample noch um Ausreißer, konkret um die jeweils fünf Unternehmen mit dem höchsten bzw. niedrigsten Umsatzwachstum. Das Sample ist nach diesen Reduzierungen zwar immer noch groß genug, um zu statistisch aussagekräftigen Ergebnissen zu kommen. Es sei allerdings betont, dass sich die folgenden Analysen nicht auf alle Unternehmen in Österreich beziehen, sondern nur auf jene Gruppe, die durch die FFG gefördert wurden. Eine Berücksichtigung der unternehmensspezifischen F\&E-Quote für alle Unternehmen Österreichs ist aufgrund der bestehenden Datenlage zurzeit nicht möglich.

Wir untersuchen die Unternehmen, die 2016 eine FFG-Förderung erhalten haben, und interessieren uns für ihre Entwicklung seit 2016. Die abhängige Variable entspricht somit stets der Entwicklung im Zeitraum 2016-2020. Die erklärenden Variablen entstammen den Daten der Aurelia-Datenbank 2017 sowie den Angaben der FFG-Datenbank im Jahr der Förderung, also 2016. Da die Aurelia-Datenbank 2017 zur Mitte des Jahres abgerufen wurde, stammen die entsprechenden Unternehmensdaten aus den Jahren 2016 oder 2017. Unsere Vorgehensweise soll erstens den Beobachtungszeitraum maximieren, da wir davon ausgehen, dass sich die erklärenden Variablen nicht unmittelbar, sondern erst mit Verzögerung auswirken. Zweitens soll sie möglichst viele Unternehmen inkludieren, um zu statistisch aussagekräftigen Ergebnissen zu kommen. 


\section{Beschäftigung}

Die erste Regression untersucht die Auswirkung verschiedener Variablen auf das prozentuale Beschäftigungswachstum. Da die abhängige Variable nicht binär, sondern metrisch ist, kommt die Methode der Kleinsten-Quadrate-Schätzer (KQS) zur Anwendung.

Wovon hängt das Beschäftigungswachstum der Unternehmen ab? In Tabelle 9 sind nur wenige Variable statistisch signifikant. Je größer das Unternehmen nach Anzahl der Beschäftigten ist, desto größer ist das anschließende Wachstum. Jüngere Unternehmen wachsen kräftiger (das Alter hat einen negativen Koeffizienten). Wichtig sind die Effekte des FFG-Fördervolumens und der unternehmensspezifischen F\&E-Quote. Der Korrelationskoeffizient der beiden (logarithmierten) Variablen beträgt 0,26568 und ist statistisch signifikant von null verschieden. Demnach ist das Fördervolumen je Beschäftigten umso höher, je mehr F\&E ein Unternehmen betreibt. Die Ergebnisse zeigen, dass das Fördervolumen je Beschäftigten positiv auf das Beschäftigungswachstum wirkt. Ein statistisch signifikanter Effekt der F\&E-Ausgaben je Mitarbeiterin bzw. Mitarbeiter auf das Beschäftigungswachstum ist jedoch nicht festzustellen.

Tabelle 9: Wachstum der Beschäftigten 2016-2020

\begin{tabular}{|l|l|l|}
\hline & Koeffizient & Standardfehler \\
\hline $\log ($ Beschäftigte) & 0,6724 & $0,3332^{* *}$ \\
\hline Kapital & $-0,0114$ & 0,1851 \\
\hline $\log$ (Alter) & $-1,4778$ & $0,4655^{* * *}$ \\
\hline Schweizer Geschäftsführer/-in & 0,6942 & 9,7410 \\
\hline Exportquote & 0,0130 & 0,0115 \\
\hline Branche C & $-1,8437$ & $0,8354^{* *}$ \\
\hline Branche F & $-0,4771$ & 1,9038 \\
\hline Branche H & $-0,9873$ & 2,4323 \\
\hline Branche N & 2,5142 & 4,0578 \\
\hline Wien & $-0,5620$ & 1,1111 \\
\hline Oberösterreich & 1,0291 & 0,7646 \\
\hline $\log$ (F\&E je Beschäftigten) & $-0,0892$ & 0,0992 \\
\hline $\log$ (FFG-Volumen je Beschäftigten) & 0,3275 & $0,1374^{* *}$ \\
\hline$A I C$ & 1824,117 & \\
\hline$B P$-Test & $23,636(0,0228)$ & \\
\hline$R^{2}$ & 0,09712 & \\
\hline$N$ & 289 & \\
\hline
\end{tabular}

Quelle: Daten wie im Text dokumentiert; Berechnung: WPZ Research

Anmerkung: KQS-Regression. Die Standardfehler geben Auskunft über die statistische Irrtumswahrscheinlichkeit, wobei * eine Wahrscheinlichkeit von $<10 \%,{ }^{* *}$ von $<5 \%$ und ${ }^{* * *}$ von $<1 \%$ bezeichnet; „log“ zeigt an, dass die Variable für die Regression logarithmiert wurde; AIC bezeichnet den Wert des Akaike-Informationskriteriums, welches die Modellgüte anzeigt (je niedriger der Wert, desto besser), BP-Test den Breusch-Pagan-test für Homoskedastizität, $n$ die Samplegröße; Branchenbezeichnungen: C: Herstellung von Waren; F: Bau; H: Verkehr und Lagerei; N: Erbringung von sonstigen wirtschaftlichen Dienstleistungen 


\section{Umsatz}

Die nächste Regression in Tabelle 10 beleuchtet die Einflüsse auf das Umsatzwachstum.

Das F\&E-Volumen in Prozent des Umsatzes hat einen sehr starken Einfluss auf das nachfolgende Umsatzwachstum. Je mehr Umsatzprozente die Unternehmen für F\&E einsetzen, desto stärker wachsen sie nachher. Zusätzlich wirkt sich die branchenspezifische F\&E-Quote (,F\&E-Quote" in der Tabelle) positiv aus. Abbildung 17 deutet darauf hin, dass Unternehmen in forschungsintensiven Branchen tendenziell schneller wachsen. Die negativen Effekte der F\&E je Beschäftigten und des FFGFördervolumens sind hier nur in Verbindung mit den anderen Variablen $\mathrm{zu}$ interpretieren. Tatsächlich ist der bloße Korrelationskoeffizient dieser beiden Variablen mit dem Umsatzwachstum jeweils statistisch nicht signifikant von null unterschiedlich.

Tabelle 10: Umsatzwachstum 2016-2020

\begin{tabular}{|l|l|l|}
\hline & Koeffizient & Standardfehler \\
\hline Umsatz je Beschäftigten & 0,0005 & $0,0001^{* * *}$ \\
\hline F\&E-Quote & 0,8163 & $0,2035^{* * *}$ \\
\hline Branche N & $-0,6474$ & $0,3054^{* *}$ \\
\hline F\&E je Beschäftigten & $-0,0024$ & $0,0008^{* * *}$ \\
\hline F\&E pro Umsatz & 0,5021 & $0,1014^{* * *}$ \\
\hline F\&E pro Kapitalstock & 0,5397 & $0,2016^{* * *}$ \\
\hline FFG-Volumen pro Umsatz & $-0,0004$ & $0,0002^{* *}$ \\
\hline$A I C$ & 311,6221 & \\
\hline$B P-T e s t$ & $6,8307(0,3368)$ & \\
\hline$R^{2}$ & 0,3771 & \\
\hline$n$ & 289 & \\
\hline
\end{tabular}

Quelle: Daten wie im Text dokumentiert; Berechnung: WPZ Research

Anmerkung: KQS-Regression. Die Standardfehler geben Auskunft über die statistische Irrtumswahrscheinlichkeit, wobei * eine Wahrscheinlichkeit von $<10 \%,{ }^{* *}$ von $<5 \%$ und ${ }^{* * *}$ von $<1 \%$ bezeichnet; AIC bezeichnet den Wert des AkaikeInformationskriteriums, welches die Modellgüte anzeigt (je niedriger der Wert, desto besser), BP-Test den Breusch-Pagantest für Homoskedastizität, $n$ die Samplegröße; Branchenbezeichnung: N: Erbringung von sonstigen wirtschaftlichen Dienstleistungen 


\section{Exportquote}

Wie beeinflusst die F\&E-Intensität die Exportneigung? Da in den FFG Daten zu wenig geeignete Beobachtungen vorhanden sind, greifen wir für diese Regression auf die Angaben zur Exportquote in der Aurelia-Datenbank zurück. ${ }^{15}$ Die Ergebnisse in Tabelle 11 zeigen einen negativen Einfluss der Exportquote zu Beginn des Beobachtungszeitraums. Je weniger ein Unternehmen 2016 exportiert hat, umso größer war das anschließende Wachstum. Positive und statistisch signifikante Effekte üben alle anderen Variablen aus. Neben der Zugehörigkeit zur Branche C (Herstellung von Waren) und einem Standort in Vorarlberg wirken die F\&E-Ausgaben je Beschäftigten positiv, und ebenso das FFGFördervolumen je Beschäftigten bzw. je Kapitalstockeinheit.

Der Breusch-Pagan-Test deutet auf Probleme mit Heteroskedastizität an, weshalb zusätzlich in Tabelle 11 gegen dieses Problem robuste Standardfehler angegeben werden, die statistischen Signifikanzniveaus ändern sich dadurch allerdings nicht. Offen bleibt allerdings, wie auskunftsfreudig Unternehmen hinsichtlich ihrer Exportquote sind: Nicht wenige geben hier im Ausgangsjahr null an, was die Ergebnisse womöglich extremer erscheinen lässt, als sie tatsächlich sind. ${ }^{16}$

Tabelle 11: Wachstum der Exportquote 2016-2020

\begin{tabular}{|l|l|l|l|}
\hline & Koeffizient & Standardfehler & „robuste Standardfehler“ \\
\hline Umsatz je Beschäftigten & 0,1615 & $0,0367^{* * *}$ & $0,0333^{* * *}$ \\
\hline Exportquote 2016 & $-4,3800$ & $0,4816^{* * *}$ & $0,5129^{* * *}$ \\
\hline Branche C & 248,6000 & $37,6900^{* * *}$ & $45,2510^{* * *}$ \\
\hline Vorarlberg & 245,1000 & $76,0300^{* * *}$ & $71,0040^{* * *}$ \\
\hline F\&E je Beschäftigten & 2,4070 & $0,4268^{* * *}$ & $0,7195^{* * *}$ \\
\hline FFG-Volumen pro Beschäftigten & 0,0013 & $0,0007^{*}$ & $0,0007^{*}$ \\
\hline FFG-Volumen pro Kapitalstock & 13,8400 & $2,9210^{* * *}$ & $3,8445^{* * *}$ \\
\hline$A I C$ & 4067,962 & & \\
\hline$B P$-Test & $53,936(0,0000)$ & & \\
\hline$R^{2}$ & 0,5135 & & \\
\hline$N$ & 289 & & \\
\hline
\end{tabular}

Quelle: Daten wie im Text dokumentiert; Berechnung: WPZ Research

Anmerkung: KQS-Regression. Die Standardfehler geben Auskunft über die statistische Irrtumswahrscheinlichkeit, wobei * eine Wahrscheinlichkeit von $<10 \%,{ }^{* *}$ von $<5 \%$ und ${ }^{* * *}$ von $<1 \%$ bezeichnet; "robuste Standardfehler" nach White; AIC bezeichnet den Wert des Akaike-Informationskriteriums, welches die Modellgüte anzeigt (je niedriger der Wert, desto besser), BP-Test den Breusch-Pagan-test für Homoskedastizität, $n$ die Samplegröße; Branchenbezeichnung: C: Herstellung von Waren

15 Die Daten hierfür wurden zwar auch von der FFG übermittelt, beziehen sich jedoch stets auf das Jahr, in dem das Unternehmen eine Förderung erhalten hat. Ein Wachstum könnte daher nur für jene Unternehmen berechnet werden, die sowohl 2016 als auch 2019 oder 2020 eine Förderung erhalten haben. Das würde das Sample nicht nur erheblich verkleinern, sondern obendrein auf jene Unternehmen beschränken, die von der FFG auch am Ende des Beobachtungszeitraums als förderungswürdig ausgewählt wurden.

16 Das eine Wachstumsrate ausgehend von null nicht definiert ist, wird hier jeweils eine Exportquote von 0,1 \% unterstellt. 


\section{Überlebensrate}

Um die Einflüsse auf die Überlebenswahrscheinlichkeit festzumachen, kommt wieder eine logistische Regression mit einer binären abhängigen Variablen zur Anwendung. Die abhängige Variable nimmt den Wert eins an, wenn das geförderte Unternehmen im Jahr 2020 in der Aurelia-Datenbank nicht mehr aufscheint, sonst null. Daher sind die Koeffizienten der erklärenden Variablen so zu lesen, dass sie die Sterbewahrscheinlichkeit erhöhen. Die Samplegröße erhöht sich, da die Entwicklung bis 2020 abgesehen von der bloßen Existenz irrelevant ist. Daher sind Unternehmen, die für 2020 keine Umsatz- oder Beschäftigtenzahlen ausweisen oder nicht mehr existieren, in das Sample wieder aufgenommen worden, ebenso die Ausreißer beim Umsatzwachstum.

Welche Unternehmenseigenschaften erhöhen die Überlebenschancen eines Unternehmens? Nach Tabelle 12 ist die Zugehörigkeit zu Branchen E (Wasserversorgung) und H (Verkehr) für die Überlebenswahrscheinlichkeiten der Unternehmen signifikant nachteilig, eine hohe Zahl an Mitarbeiterinnen und Mitarbeitern ist hingegen vorteilhaft. Die forschungsbezogenen Variablen sind statistisch nicht signifikant. Allerdings schrammt das FFG-Fördervolumen je Beschäftigten knapp am 10\%-Niveau vorbei (Irrtumswahrscheinlichkeit 12,16\%). Das Vorzeichen ist hier negativ, d.h. die Überlebenschance wird durch die Höhe des Fördervolumens tendenziell begünstigt. Bei allen statistischen Vorbehalten wäre davon auszugehen, dass eine FFG-Förderung tatsächlich eher die Unternehmen mit den besseren Erfolgschancen erreicht.

\section{Tabelle 12: Überlebenswahrscheinlichkeiten}

\begin{tabular}{|l|l|l|}
\hline & Koeffizient & Standardfehler \\
\hline Kapitalstock & $>0.0000$ & 0.0000 \\
\hline Branche E & 2.4690 & $1.4560^{*}$ \\
\hline Branche H & 2.1100 & $1.0120^{* *}$ \\
\hline Branche K & 15.5500 & 882.7000 \\
\hline Branche M & 0.3065 & 0.4230 \\
\hline log(Anzahl Beschäftigte) & -0.6635 & $0.0796^{* * *}$ \\
\hline F\&E je Beschäftigten & -0.0071 & 0.0071 \\
\hline FFG-Volumen pro Beschäftigten & $<0.0000$ & 0.0000 \\
\hline$A I C$ & 193.381 & \\
\hline$n$ & 353 & \\
\hline
\end{tabular}

Quelle: Daten wie im Text dokumentiert; Berechnung: WPZ Research

Anmerkung: Logit-Regression. Abhängige Variable ist „Unternehmen existiert 2020 nicht mehr“ (=1 wenn ja). Die Standardfehler geben Auskunft über die statistische Irrtumswahrscheinlichkeit, wobei * eine Wahrscheinlichkeit von $<10 \%$, ** von $<5 \%$ und ${ }^{* * *}$ von $<1 \%$ bezeichnet; "log“ zeigt an, dass die Variable für die Regression logarithmiert wurde; AIC bezeichnet den Wert des Akaike-Informationskriteriums, welches die Modellgüte anzeigt (je niedriger der Wert, desto besser), BP-Test den Breusch-Pagan-test für Homoskedastizität, $n$ die Samplegröße; Branchenbezeichnungen: E: Wasserversorgung; Abwasser- und Abfallentsorgung und Beseitigung von Umweltverschmutzungen; H: Verkehr und Lagerei; K: Erbringung von Finanz- und Versicherungsdienstleistungen; M: Erbringung von freiberufl., wissenschaftl. und techn. Dienstleistungen 


\section{Zwischenfazit}

Die Ergebnisse für das Sample der im Jahr 2016 geförderter Unternehmen zeigen insgesamt deutlich positive Effekte der unternehmensspezifischen F\&E-Ausgaben sowie des Fördervolumens durch die FFG. Die Einflüsse auf die Wachstumsraten der Beschäftigung, des Umsatzes und der Exportquote fallen jedoch recht unterschiedlich aus. Beim Beschäftigungswachstum ist das FFG-Fördervolumen pro Beschäftigten am aussagekräftigsten. Beim Umsatzwachstum sind es die F\&E-Quoten in Prozenten des Umsatzes und des Kapitalstocks. Die Zunahme der Exportquoten hängt ab von den F\&E-Ausgaben je Beschäftigten, vom FFG-Fördervolumen pro Beschäftigten und vom FFG-Volumen pro Einheiten des Kapitalstocks. Deutliche Effekte zeigen fallweise auch die Branchenzugehörigkeiten.

Bei der Interpretation der Ergebnisse ist zu berücksichtigen, dass die Analyse nur einen kleinen Ausschnitt aller durch die FFG geförderten Unternehmen berücksichtigen konnte, und dass die FFG ihrerseits besonders forschungsintensive Unternehmen fördert. Außerdem konnte die Analyse des Unternehmenswachstums nur jene erfassen, die den Beobachtungszeitraum tatsächlich überlebt haben. Die Ergebnisse stehen insgesamt mit der internationalen empirischen Evidenz im Einklang und geben zusätzliche Hinweise für Österreich, dass F\&E das Unternehmenswachstum begünstigt.

\section{Interpretation der Ergebnisse im internationalen Kontext}

Wie sind diese Ergebnisse im Kontext internationaler Forschungsarbeiten zu interpretieren? Zunächst einmal zeigen die Ergebnisse eine Präferenz der FFG für das verarbeitende Gewerbe im Allgemeinen und forschungsintensive Branchen im Besonderen. Somit kann ein positiver Effekt, der bspw. von Donselaar und Koopmans (2016) auf die Umsatzsteigerung festgestellt wird, jedenfalls nicht ausgeschlossen werden, da der Umsatz - wie in Abbildung 18 dargestellt - positiv mit einer F\&EFörderung korreliert ist.

Hinsichtlich Risikokapitals zeigt Howell (2017) anhand des Programms SBIR (Small Business Innovation Research) des US-amerikanischen Energieministeriums, dass nach einer Förderung durch SBIR die Wahrscheinlichkeit, Wagniskapital zu erhalten, sprunghaft von $10 \%$ auf $20 \%$ ansteigt. Wagniskapital ist insbesondere für junge Unternehmen wichtig, die aufgrund hoch riskanter Produkte oder Geschäftsmodelle keine Finanzierung von Banken erhalten. Wagniskapitalgeberinnen und -geber tragen außerdem wesentlich zur Professionalisierung ihrer Beteiligungsfirmen bei, denn sie wählen aus vielen Kandidaten nur wenige, besonders innovative Unternehmen aus, stellen ein Netzwerk an Geschäftskontakten zur Verfügung und bringen neue Technologien schneller auf den Markt, um das Wachstumspotential zu steigern. Insofern sind die Ergebnisse aus Tabelle 8 von besonderer Relevanz, da eine Wechselwirkung zwischen FFG-Förderung und Risikokapital (von dem Wagniskapital ein Teil ist) offenbar besteht. Nicht zuletzt aufgrund des chronischen Mangels in Österreich an Wagniskapital ist dieses Ergebnis relevant.

Görg und Strobl (2007) gehen ebenfalls der Frage nach, in welcher Form direkte Forschungsförderungen die größte Wirkung entfaltet. Dazu nutzen sie Daten zu irischen Produktionsbetrieben im Zeitraum von 1998 bis 2002 und unterscheiden zwischen niedrigen (bis 12.500 Euro), mittleren (zwischen 12.500 und 55.000 Euro) und hohen F\&E-Förderungen (über 55.000 Euro). Es zeigt sich, dass niedrige F\&E-Förderungen die unternehmerischen F\&E-Ausgaben anregen, bei den hohen Förderungen ist allerdings ein Crowding-Out-Effekt zu beobachten: Die hohen öffentlichen F\&EAusgaben verdrängen die privaten F\&E-Ausgaben. Da sich die Förderungen der FFG hinsichtlich ihres Volumens stark unterscheiden, wäre auch hier eine tiefergehende Analyse angezeigt.

Abbildung 17 zeigt einen positiven Zusammenhang zwischen FFG-Förderungen und anschließendem Wachstum der Beschäftigtenzahlen. Dass F\&E und daraus folgende Innovationen die Beschäftigung eines Unternehmens erhöhen, ist Ergebnis zahlreicher Studien, darunter Harrison et al. (2014), die mehr als 20.000 Unternehmen in Frankreich, Deutschland, Spanien und Großbritannien untersuchen. 
Dabei zeigt sich, dass Unternehmen mit Produktinnovationen in allen vier Ländern eine höhere Produktivität aufweisen und höhere Umsätze erzielen als jene Unternehmen, die keine Innovationen hervorbringen. In puncto Beschäftigung können grundsätzlich zwei Effekte identifiziert werden: Einerseits kann höhere Produktivität dazu führen, dass die Beschäftigung reduziert wird, anderseits kann eine erhöhte Produktivität zu niedrigeren Preisen führen, die die Nachfrage und schließlich die Beschäftigung erhöhen. Harrison et al. (2014) stellen fest, dass Produktinnovationen durch den Nachfrageeffekt mehr Arbeitsplätze schaffen als durch den Produktivitäts- und Substitutionseffekt zwischen dem alten und dem neuen Produkt vernichtet werden. Moretti und Wilson (2014) analysieren neben anderen Effekten die Beschäftigungseffekte von F\&E im Biotechnologiesektor in den USA und stellen im Allgemeinen und auch hier deutlich positive Wirkungen der F\&E-Förderung fest.

Die Ergebnisse zeigen auch einen positiven Zusammenhang der FFG-Förderung mit den Exportquoten der Unternehmen an. Maican et al. (2020) analysieren in einer ähnlichen Fragestellung, inwiefern Exporte und F\&E zusammenhängen und untersuchen dazu schwedische Unternehmen im Zeitraum von 2003 bis 2010. Mit einem relativ kleinen Inlandsmarkt sind schwedische Unternehmen, ebenso wie österreichische, stark auf Verkäufe auf ausländischen Märkten angewiesen. Es wird gezeigt, dass Investitionen in F\&E auf den Exportmärkten einen größeren Einfluss auf die Einnahmen und Gewinne der Unternehmen haben als auf dem Inlandsmarkt (Maican et al., 2020). Tabelle 12 zeigt, dass ein Zusammenhang zwischen F\&E und Steigerung der Exportquote in Österreich zumindest für FFG-geförderte Unternehmen besteht. 


\section{Schlussfolgerungen}

Wie groß sind die Wirkungen der öffentlichen F\&E-Ausgaben? Die Fragestellung meint sowohl die Wirkungen auf die Gesamtwirtschaft als auch auf die Entwicklung auf Unternehmensebene. Eine belastbare Antwort zu geben, ist keine triviale Angelegenheit. Mit dem Einsatz des WPZ Innovationsmodells können wir folgendes Ergebnis festhalten: Ein Euro an öffentlichen Mehrausgaben für die Forschung führt zu einem langfristigen BIP-Zuwachs von 6 Euro. In einem Wachstumsszenario nach dem Muster der vergangenen zehn Jahre sind etwa $28 \%$ des kumulativen Wachstums auf die Effekte des heimischen Innovationssystems zurückzuführen. Mit öffentlicher F\&E-Förderung, in Österreich sind es vor allem die Forschungsprämie und die direkten Subventionen der FFG, setzt der Staat wichtige Impulse für mehr private F\&E-Investitionen. Wie bei allen quantitativen Methoden können die Ergebnisse innerhalb gewisser Bandbreiten schwanken und sind nicht als punktgenaue Schätzungen zu verstehen.

Um die Auswirkungen auf Unternehmensebene festzumachen, vergleichen wir mit ökonometrischen Methoden die Entwicklung von geförderten Unternehmen relativ zu nicht-geförderten, aber sonst ganz ähnlichen Unternehmen. Das Ergebnis ist, dass die FFG-geförderten Unternehmen ihre Beschäftigung im Zeitraum 2016-2019 um 7,1\% steigern konnten, andere vergleichbare Unternehmen dagegen nur um $0,4 \%$. Das Umsatzwachstum beträgt 10,8\% statt 2,5\%. Auch in anderen Dimensionen des Unternehmenserfolgs sind F\&E intensive Unternehmen den Konkurrenten überlegen: Sie sind erfolgreicher, Wagnis- und Risikokapital anzuziehen, was die Voraussetzung für eine weitere Finanzierung schafft und meist einen Meilenstein für die Unternehmensentwicklung darstellt. Sie haben tendenziell eine höhere Überlebensrate, denn mit F\&E bauen sie ihre Wettbewerbsvorteile aus und sind auf dem Markt erfolgreicher. Dank einem Qualitätsvorsprung durch Innovation können sie trotz hoher Lohnkosten und weltweiter Konkurrenz in die internationalen Absatzmärkte vorstoßen und haben daher höhere Exportquoten. Eine hohe F\&EIntensität schlägt sich über kurz oder lang in einen höheren Innovationsgehalt der F\&E-Projekte nieder, so dass innovative Unternehmen häufiger als andere den Wettbewerb um kompetitiv vergebene FFG-Fördermittel gewinnen.

F\&E-Ausgaben haben eine außerordentlich hohe volkswirtschaftliche Rendite, welche die private Rendite der Unternehmen bei weitem übersteigt. Die Unternehmen investieren in F\&E, weil sie ihre Wettbewerbsfähigkeit ausbauen, Marktanteile gewinnen und so die künftigen Gewinne steigern wollen. Dabei zeitigen die Investitionen der einzelnen Unternehmen eine zusätzliche Rendite für die gesamte Volkswirtschaft. Die Investitionen der Unternehmen bauen auf den Erfahrungen und Erkenntnissen anderer Unternehmen auf. In der Innovation ist das Ganze eben mehr als die Summe der Teile. Um die Überschussrendite der privaten Innovation zu realisieren, sollte der Staat mit steuerlichen Anreizen und direkten Subventionen mehr private F\&E-Investitionen anstoßen (Korrektur eines Marktversagens). Die Ausgaben für F\&E sind eine zentrale Vorleistung für private Innovationen. Wie bei anderen öffentlichen Gütern muss der Staat den Löwenanteil finanzieren, um die Voraussetzungen für private Innovation zu schaffen. Ein investiver Staat muss die Budgetmittel dorthin lenken, wo sie für die Gesellschaft den höchsten Ertrag erwirtschaften. So wie sich die Situation in Österreich präsentiert, kann der Staat mit erheblichen Mehrausgaben für F\&E die Voraussetzungen für nachhaltiges Wachstum wesentlich verbessern.

Angesichts der großen gesellschaftlichen Herausforderungen wie der Klimakrise oder der Digitalisierung gilt es zudem, die Grundlagenforschung zu stärken und strategische Prioritäten in der FTI-Politik zu setzen, damit das heimische Innovationssystem zur Bewältigung und Lösung beitragen und damit Wachstum und künftigen Wohlstand sichern kann. Eine besondere Situation schafft die aktuelle COVID-19-Pandemie. Es ist absehbar, dass die F\&E-Ausgaben des Unternehmenssektors, der für knapp die Hälfte $(47,6 \%)$ der österreichischen F\&E-Ausgaben verantwortlich ist, drastisch zurückgehen werden. Umso kritischer ist es, dass der öffentliche Sektor durch langfristige öffentliche Finanzierung einen Rückfall in der Innovationsperformance verhindert. Einsparungen bei den 
öffentlichen Forschungsausgaben im Zuge des Abbaus der COVID-19-Schulden würde der Wettbewerbsfähigkeit der Wirtschaft und dem Wachstumspotential Österreichs nachhaltig schaden und das Aufholen nach der Krise wesentlich erschweren und verzögern.

Die Innovationspolitik braucht allerdings einen langen Atem. Zwar steigen die Wachstumsraten relativ rasch an, aber es braucht viel Zeit, bis die kumulativen Effekte auf den mit F\&E geschaffenen Wissensbestand und damit die möglichen Produktivitätssteigerungen vollständig eintreten. Das ist für die Wirtschaftspolitik eine Herausforderung. Die F\&E-Ausgaben müssen sofort erhöht werden, die positiven volkswirtschaftlichen Wirkungen treten dagegen nur langsam ein. Auch im Unternehmenssektor haben F\&E-Investitionen eine lange Vorlaufzeit. Es dauert, bis die höheren Gewinne fließen. In der Grundlagenforschung sind die Vorlaufzeiten noch viel länger. So gilt auch in der Innovationspolitik: Wer Technologieführerschaft erreichen will, muss zuerst investieren. 


\section{Literatur}

Acemoglu, D. (2009), Introduction to Modern Economic Growth, Princeton University Press.

Aghion, P. und Howitt, P. (1992), A Model of Growth through Creative Destruction, Econometrica, 60, 323-351.

Aghion, P. und Howitt, P. (1998), Endogenous Growth Theory, MIT Press.

Aghion, P. und Howitt, P. (2009), The Economics of Growth, MIT Press.

Akcigit, Caicedo, S., Miguelez, E., Stantcheva, S. und Sterzi, V. (2018), Dancing with the Stars: Innovation Through Interactions, NBER WP 24466.

Akcigit, U. und Stantcheva, S. (2020), Taxation and Innovation: What do We Know?, NBER WP 27109.

Akcigit, U., Hanley, D. und Serrano-Velarde, N. (2013), Back to Basics: Basic Research Spillovers, Innovation Policy and Growth, NBER WP 19473.

Anzoategui, D., Comin, D., Gertler, M. und Martinez, J. (2019), Endogenous technology adoption and R\&D as sources of business cycle persistence, American Economic Journal: Macroeconomics, 11(3), 67-110.

Aristei, D., Sterlacchini, A. und Venturini, F. (2017), Effectiveness of R\&D subsidies during the crisis: firm-level evidence across EU countries, Economics of Innovation and New Technology, 26(6), 554573.

Arrow, K.J. (1962), Economic Welfare and the Allocation of Resources to Invention, In: The Rate and Direction of Inventive Activity, Princeton University Press.

Barlevy, G., (2007), On the Cyclicality of Research and Development, The American Economic Review, 97(4), 1131-1164.

Belitz, H, Junker, S., Schiersch, A. und Podstawski, M. (2015), Wirkung von Forschung und Entwicklung auf das Wirtschaftswachstum, DIW, Berlin.

Best, H. und Wolf, C. (2010), Logistische Regression, in: Best, H. und Wolf, C. (Hrsg.): Handbuch der sozialwissenschaftlichen Datenanalyse. Wiesbaden, VS Verlag, 827-854.

Bittschi, B., Fortin, I., Grozea-Helmenstein, D., Hlouskova, J., Hofer, H., Koch, S. P., Kocher, M., Kunst, R., Reiter, M., Sellner, R., und Weyerstrass, K., (2020), Prognose der österreichischen Wirtschaft 20202021: Zögerliche Erholung von der COVID-19-Krise, IHS-Wirtschaftsprognose 114, 47.

BKA (2020), Aus Verantwortung für Österreich - Regierungsprogramm 2020-2024, Wien.

Bloom, N., Griffith, R. und Van Reenen, J. (2002), Do R\&D tax credits work? Evidence from a panel of countries 1979-1997, Journal of Public Economics, 85 (1), 1-31.

Bloom, N., Jones, C. I., Van Reenen, J. und Webb, M. (2020), Are Ideas Getting Harder to Find?, American Economic Review, 110 (4), 1104-44.

Bloom, N., Schankerman, M.A. und Van Reenen, J. (2013), Identifying technology spillovers and product market rivalry, Econometrica, 81(4), 1347-1393.

BMBWF, BMK und BMDW (2020), Österreichischer Forschungs- und Technologiebericht 2020, Wien.

BMBWF, BMVIT und BMDW (2019), Österreichischer Forschungs- und Technologiebericht 2019, Wien.

BMF (2020), Budgetbericht 2021, Wien.

Boeing, P. und Hünermund, P. (2020), A global decline in research productivity? Evidence from China and Germany, Economics Letters, 197, 109646. 
Bravo-Biosca, A., Martson, L., Mettler, A., Mulgan, G. und Westlake S. (2013), Plan I - Innovation for Europe, Nesta and The Lisbon Council.

Czarnitzki, D. und Hussinger, K. (2018), Input and output additionality of R\&D subsidies, Applied Economics, 50(12), 1324-1341,

Donselaar, P. und Koopmans C. (2016), The Fruits of R\&D: Meta-analysis of the Effects of Research and Development on Productivity, Research Memorandum, No. 2016-1, Vrije University, Amsterdam.

Ecker, B., Brandl, B., Fink., N., Kaufmann, P., Loretz, S., Sardadvar, S., Sellner, R., Sheikh, S. und Wolf, L. (2017), Evaluierung der Forschungsprämie gem. § 108c EStG, Wien.

EFI - Expertenkommission Forschung und Innovation (2016), Gutachten zu Forschung, Innovation und technologischer Leistungsfähigkeit Deutschland, Patentboxen - Kein Ersatz für steuerliche FuEFörderung, Berlin.

Egger, P. und Keuschnigg, C. (2015), Innovation, Trade and Finance, American Economic Journal: Microeconomics 7, 121-157.

Europäische Kommission (2010), Europa 2020 - Eine Strategie für intelligentes, nachhaltiges und integratives Wachstum, Brüssel.

Europäische Kommission (2017), The Economic Rationale for Public R\&I Funding and Its Impact, Policy Brief Series, Directorate-General for Research and Innovation.

Europäische Kommission (2020a), Ein neuer EFR für Forschung und Innovation, COM(2020) 628, Brüssel.

Europäische Kommission (2020b), Investitionsplan für ein zukunftsfähiges Europa -Investitionsplan für den europäischen Grünen Deal, $\operatorname{COM(2020)~21,~Brüssel.~}$

Europäische Kommission (2020c), Science, Research and Innovation Performance of the EU 2020 - A fair, green and digital Europe, Brüssel

Europäische Kommission (2020d), Shaping Europe's digital future, Brüssel.

Falk, M. und Hake, M. (2008), Wachstumswirkungen der Forschungsausgaben, WIFO, Wien.

Farre-Mensa J., Hegde, D. und Ljungqvist, A.P. (2016), The Bright Side of Patents, CEPR DP No. 11091.

Fenz, G. und Schneider, M. (2020), Gesamtwirtschaftliche Prognose der OeNB für Österreich 2020 bis 2023 - Zweite COVID-19-Welle verzögert Konjunkturerholung, Österreichische Nationalbank, Wien.

Friesenbichler, K.S., Janger, J., Kügler, A. und Reinstaller, A. (2020), Auswirkungen der COVID-19Pandemie auf die Forschungs- und Innovationsaktivität, WIFO, Wien.

Gogola, G. (2020), Forschung und Entwicklung in der COVID-19-Pandemie - Innovation stärkt die Krisenfestigkeit, WPZ Research Policy Brief 1/20, Wien.

Görg, H. und Strobl, E. (2007), The Effect of R\&D Subsidies on Private R\&D, Economica, 74 (294), 215234

Griffith, R., Redding, S. und Van Reenen, J. (2004), Mapping the two faces of R\&D: productivity growth in a panel of OECD countries, Review of Economics and Statistics, 86(4), 883-895.

Grossman, G. M. und Helpman, E. (1991a): Quality ladders in the theory of growth, Review of Economic Studies, 68, 43-61.

Grossman, G. M. und Helpman, E. (1991b): Innovation and growth in the global economy. MIT Press

Guellec, D. und van Pottelsberghe de La Potterie, B. (2004), $R \& D$ and productivity growth: panel data analysis of 16 OECD countries, OECD Economic Studies, 33. 
Hall, B. H. (2002), The Financing of Research and Development, Oxford Review of Economic Policy, 18 (1), 35-51.

Hall, B. H. (2011), Innovation and Productivity, NBER Working Paper 17178.

Harrison, R., Jaumandreu, J., Mairesse, J. und Peters, B. (2014), Does innovation stimulate employment? A firm-level analysis using comparable micro-data from four European countries, International Journal of Industrial Organization, 35, 29-43.

Health Economics Research Group, Office of Health Economics und RAND Europe (2008), Medical Research: What's it worth? Estimating the economic benefits from medical research in the UK, UK Evaluation Forum, London.

Howell, S. T. (2017), Financing Innovation: Evidence from R\&D Grants, American Economic Review, 107(4), 1136-1164.

Keuschnigg, C. (1994), Venture Capital Backed Growth, Journal of Economic Growth 9, 239-261.

Keuschnigg, C. (2016), ed., Moving to the Innovation Frontier, Ebook, Centre for Economic Policy Research, London.

Keuschnigg, C. und Matt, J. I (2020), An Innovation Based Growth Model, Technical Model Documentation.

Keuschnigg, C. und Sardadvar, S. (2019), Wagniskapital zur Finanzierung von Innovation und Wachstum, Studie von WPZ, St. Gallen, und WPZ Research, Wien, im Auftrag des RFTE und der AVCO.

Keuschnigg, C., Ecker, B., Sardadvar, S. und Reiner, C. (2017), Innovationsland Österreich: F\&E, Unternehmensentwicklung und Standortattraktivität, Wirtschaftspolitisches Zentrum, Studie im Auftrag des Rats für Forschung und Technologieentwicklung.

Lucking, B., Bloom, N. und Van Reenen, J. (2018), Have R\&D Spillovers Changed?, NBER WP 24622.

Maican, F. G., Orth, M., Roberts, M. J. und Van Anh, V. (2020), The Dynamic Impact of Exporting on Firm R\&D Investment, NBER Working Paper 27986.

Maradana, R. P, Pradhan, R. P., Dash, S., Gaurav, K., Jayakumarm M. und Chatterjee, D. (2017), Does Innovation Promote Economic Growth? Evidence from European Countries, Journal of Innovation and Entrepreneurship, 6, 1-23.

Markman, G., Balkin, D. und Schjoedt, L. (2001), Governing the innovation process in entrepreneurial firms, The Journal of High Technology Management Research, 12 (2), 273-293.

Mazzucato, M. (2013), The Entrepreneurial State: Debunking Public vs. Private Sector Myths, Anthem Press, London.

Mazzucato, M. (2018), Mission-Oriented Research \& Innovation in the European Union - A problemsolving approach to fuel innovation-led growth, Brüssel.

Moretti, E. und Wilson, D. J. (2014), State incentives for innovation, star scientists and jobs: Evidence from biotech, Journal of Urban Economics, 79(C), 20-38.

OECD (1999), Managing National Innovation Systems, Paris.

Ortega-Argilés, R., Piva, M., Potters, L. und Vivarelli, M. (2010), Is corporate R\&D investment in hightech sectors more effective?, Contemporary Economic Policy, 28(3), 353-365.

Romer, P. (1986), Increasing Returns and Long-Run Growth, Journal of Political Economy, 94, 10021037. 
Sardadvar, S. (2018), Is a company's access to private equity and venture capital affected by location or management diversity? Some empirical evidence from Austria, International Journal of Business and Management, 13(7), 45-57.

Sardadvar, S. (2019), Das Humankapital Österreichs konzentriert sich zunehmend im Osten: Ursachen, Entwicklungen und Auswirkungen, in: Bußjäger, P., Keuschnigg, G. und Schramek, C. (Hrsg.): Raum neu denken - Von der Digitalisierung zur Dezentralisierung. Innsbruck, Institut für Föderalismus, 55-70.

Sardadvar, S. (2020), Wagniskapitalfinanzierung in Österreich: der Standort Wien als großer Profiteur, WPZ Research Policy Brief 3/2020, Wien.

Schiman, S. (2020), Zähe Konjunktur nach kräftigem Rebound - Prognose für 2020 und 2021, WIFOMonatsberichte, 93(10), 715-728.

Schumpeter, J., (1950), Capitalism, Socialism and Democracy, Harper, New York.

Solow, R. M. (1956), A Contribution to the Theory of Economic Growth, The Quarterly Journal of Economics, 70, 65-94.

Solow, R. M. (1957), Technical Change and the Aggregate Production Function, Review of Economics and Statistics, 39, 312-320.

Toole, A. (2012), The impact of public basic research on industrial innovation: Evidence from the pharmaceutical industry, Research Policy, 41 (1), 1-12.

Van Elk, R., Verspagen, B., Ter Weel, B., Van der Wiel, K., und Wouterse, B. (2015), A macroeconomic analysis of the returns to public R\&D investments, CPB Netherlands Bureau for Economic Policy Analysis, 313.

Von der Leyen, U. (2019), Eine Union, die mehr erreichen will: Meine Agenda für Europa - Politische Leitlinien für die zukünftige Europäische Kommission 2019-2024, Brüssel.

Voßkamp, R. und Schmidt-Ehmcke, J. (2006), Wachstum durch Innovationen: Herausforderungen für die Innovationspolitik, DIW Wochenbericht 22127, Berlin. 


\section{Anhang I: Ergänzungen}

Basis-, Referenz- und Kontrollgruppen, verarbeitendes Gewerbe

\begin{tabular}{|c|c|c|c|}
\hline & Basis & Referenz & Kontroll \\
\hline Beschäftigte 2017 (Tsd.) & 70,45 & 104,78 & 97,89 \\
\hline Beschäftigte 2020 (Tsd.)* & 73,87 & 109,97 & 99,58 \\
\hline Umsatz 2017 (Tsd. €) & 19935,00 & 30312,74 & 34272,09 \\
\hline Umsatz 2020 (Tsd. €)* & 23177,23 & 35144,12 & 37255,69 \\
\hline Börsennotierung (=1, wenn ja) & $0,15 \%$ & $0,00 \%$ & $0,00 \%$ \\
\hline Alter seit Gründung & 29,41 & 30,67 & 31,97 \\
\hline Alter seit Umgründung & 19,87 & 21,33 & 22,11 \\
\hline Eigenkapital (Tsd. €) & 663846,59 & 996706,96 & 894490,45 \\
\hline Umsatz je Mitarbeiter/-in 2017 (Tsd. €) & 254,00 & 249,90 & 296,10 \\
\hline Umsatz je Mitarbeiter/-in 2020 (Tsd. €)* & 363,58 & 355,87 & 459,74 \\
\hline Unternehmen in Konzerngruppe & 29,73 & 42,28 & 59,49 \\
\hline Anzahl Gesellschafter & 1,92 & 2,13 & 1,91 \\
\hline Anzahl Tochtergesellschaften & 0,61 & 0,83 & 1,09 \\
\hline Anzahl Geschäftsführer/-innen & 1,49 & 1,74 & 1,67 \\
\hline Anteil österr. Geschäftsführer/-innen & $95,56 \%$ & $95,14 \%$ & $93,99 \%$ \\
\hline Anteil dt. Geschäftsführer/-innen & $2,80 \%$ & $3,02 \%$ & $3,54 \%$ \\
\hline Anteil schweiz. Geschäftsführer/-innen & $0,48 \%$ & $0,61 \%$ & $0,72 \%$ \\
\hline Anteil and. Geschäftsführer/-innen & $1,31 \%$ & $1,80 \%$ & $1,96 \%$ \\
\hline Importquote (in \%) & $15,07 \%$ & $20,86 \%$ & $23,25 \%$ \\
\hline Exportquote (in \%) & $17,78 \%$ & $36,67 \%$ & $39,15 \%$ \\
\hline ausl. Gesellschafter (=1, wenn ja) & $12,42 \%$ & $18,07 \%$ & $19,83 \%$ \\
\hline F\&E-Quote & $5,71 \%$ & $8,11 \%$ & $7,51 \%$ \\
\hline Wien & $10,27 \%$ & $5,65 \%$ & $8,38 \%$ \\
\hline Niederösterreich & $17,26 \%$ & $17,44 \%$ & $15,23 \%$ \\
\hline Burgenland & $2,68 \%$ & $2,51 \%$ & $1,89 \%$ \\
\hline Steiermark & $13,54 \%$ & $15,68 \%$ & $15,70 \%$ \\
\hline Kärnten & $6,76 \%$ & $8,16 \%$ & $8,50 \%$ \\
\hline Tirol & $8,62 \%$ & $8,03 \%$ & $7,32 \%$ \\
\hline Oberösterreich & $26,34 \%$ & $28,48 \%$ & $31,17 \%$ \\
\hline Salzburg & $7,10 \%$ & $5,65 \%$ & $4,96 \%$ \\
\hline Vorarlberg & $7,25 \%$ & $8,28 \%$ & $6,85 \%$ \\
\hline Risikokapital & $0,46 \%$ & $1,88 \%$ & $0,83 \%$ \\
\hline FFG 2017* & $6,10 \%$ & $30,11 \%$ & $2,24 \%$ \\
\hline FFG 2018* & $6,20 \%$ & $29,61 \%$ & $2,72 \%$ \\
\hline FFG 2019* & $5,52 \%$ & $26,10 \%$ & $1,89 \%$ \\
\hline FFG 2020* & $4,49 \%$ & $18,95 \%$ & $1,89 \%$ \\
\hline$N$ & 5903 & 797 & 847 \\
\hline
\end{tabular}

Arithmetische Mittelwerte. Daten wie im Text dokumentiert; Berechnung: WPZ Research.

Anmerkungen: *nicht als Variable im PSM-Verfahren berücksichtigt, sondern analog zu Tabelle 5 die Entwicklung in den Folgejahren, $n$ bezeichnet die Anzahl der Beobachtungen 
Länderkürzel

\begin{tabular}{|c|c|c|c|c|c|}
\hline Land & Kürzel & Land & Kürzel & Land & Kürzel \\
\hline Albanien & ALB & Hongkong & HKG & Norwegen & NOR \\
\hline Argentinien & ARG & Kroatien & HRV & Neuseeland & NZL \\
\hline Österreich & AUT & Ungarn & HUN & Polen & $\mathrm{POL}$ \\
\hline Australien & AUS & Irland & IRL & Portugal & PRT \\
\hline Belgien & BEL & Indien & IND & Rumänien & ROU \\
\hline Bulgarien & $B G R$ & Israel & ISR & Serbien & SRB \\
\hline Brasilien & BRA & Island & ISL & Russland & RUS \\
\hline Kanada & CAN & Italien & ITA & Schweden & SWE \\
\hline Schweiz & $\mathrm{CHE}$ & Japan & JPN & Schweiz & $\mathrm{CHE}$ \\
\hline Chile & $\mathrm{CHL}$ & Südkorea & KOR & Singapur & SGP \\
\hline China & $\mathrm{CHN}$ & Liechtenstein & LIE & Slowenien & SVN \\
\hline Zypern & CYP & Litauen & LTU & Slowakei & SVK \\
\hline $\begin{array}{l}\text { Tschechische } \\
\text { Republik }\end{array}$ & CZE & Luxemburg & LUX & Spanien & ESP \\
\hline Deutschland & DEU & Lettland & LVA & Türkei & TUR \\
\hline Dänemark & DNK & Montenegro & MNE & Taiwan & TWN \\
\hline Estland & EST & Mazedonien & MKD & Ukraine & UKR \\
\hline Griechenland & GRC & Malta & MLT & $\begin{array}{l}\text { Vereinigtes } \\
\text { Königreich }\end{array}$ & UK \\
\hline Spanien & ESP & Mexiko & MEX & $\begin{array}{l}\text { Vereinigte Staaten } \\
\text { von Amerika }\end{array}$ & USA \\
\hline Finnland & FIN & Nigeria & NGA & Südafrika & $\mathrm{ZAF}$ \\
\hline Frankreich & FRA & Niederlande & NLD & & \\
\hline
\end{tabular}




\section{Anhang II: Verzeichnisse}

\section{Tabellenverzeichnis}

Tabelle 1: Anteil der öffentlichen F\&E-Ausgaben am BIP, 2008 bis 2019 ................................................17

Tabelle 2: Langfristige Effekte öffentlicher F\&E-Ausgaben ........................................................................ 26

Tabelle 3: Langfristige Wachstumsbeiträge und makroökonomische Auswirkungen ............................. 34

Tabelle 4: Arithmetische Mittelwerte der Basis-, Referenz- und der Kontrollgruppen nach Propensity-

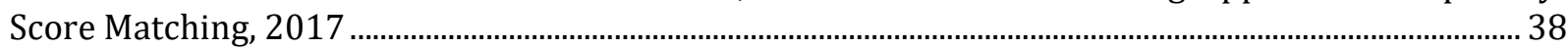

Tabelle 5: Arithmetische Mittelwerte der Basis-, Referenz- und der Kontrollgruppen in den

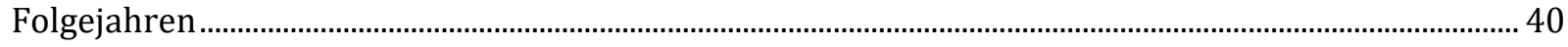

Tabelle 6: Überlebensraten der geförderten und nicht geförderten Unternehmen ................................ 44

Tabelle 7: Logit-Regression, abhängige Variable: FFG-Förderung 2010-2020 .......................................... 45

Tabelle 8: Logit-Regression, abhängige Variable: FFG-Förderung 2020 ................................................. 47

Tabelle 9: Wachstum der Beschäftigten 2016-2020 …………..................................................................... 49

Tabelle 10: Umsatzwachstum 2016-2020 …………..................................................................................... 50

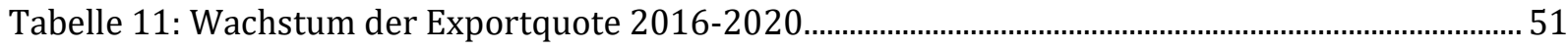

Tabelle 12: Überlebenswahrscheinlichkeiten................................................................................................. 52

\section{Abbildungsverzeichnis}

Abbildung 1: Akteure und Interaktionen im Innovationssystem ................................................................ 5

Abbildung 2: Beitrag von F\&E zum Wirtschaftswachstum in Europa, 2010-2016 .................................... 7

Abbildung 3: Beitrag von F\&E zum Arbeitsproduktivitätswachstum in Europa, 2010-2016 .................. 8

Abbildung 4: F\&E-Quote in der EU und ausgewählten Vergleichsländern, 2009 und 2019 .................. 10

Abbildung 5: F\&E-Personal und ForscherInnen (VZÄ) an der Erwerbsbevölkerung, 2008

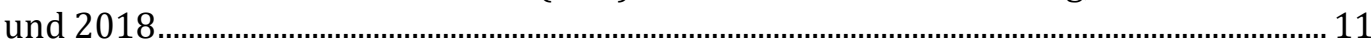

Abbildung 6: Anteil der öffentlichen F\&E-Ausgaben am BIP, 2008 und 2018 …..................................... 13

Abbildung 7: Direkte und indirekte F\&E-Förderung als Anteil des BIP, 2017 ......................................... 15

Abbildung 8: Entwicklung F\&E-Ausgaben und F\&E-Quote in Österreich, 2009-2019........................... 16

Abbildung 9: F\&E-Ausgaben nach Sektoren und Forschungsarten, Mio. Euro, 2017 .............................. 17

Abbildung 10: Steuergutschrift an Unternehmen via Forschungsprämie, 2010-2019 .......................... 20

Abbildung 11: Langfristiger BIP-Zuwachs pro Euro öffentlicher Forschungsausgaben.......................... 24

Abbildung 12: Dynamische Wirkungen öffentlicher F\&E-Ausgaben ......................................................... 28

Abbildung 13: Differentielle, jährliche Wachstumsraten des BIP, in Prozentpunkten ............................ 29

Abbildung 14: Langfristige Wachstumsbeiträge der Innovation .................................................................. 32 
Abbildung 15: Dynamische Wachstumsbeiträge der Innovation 35

Abbildung 16: Wachstum von Beschäftigung und Umsatz, 2016-2019.. 41

Abbildung 17: Wachstum der Beschäftigten nach Branchen, 2016-2019 42

Abbildung 18: Wachstumsraten der Umsätze nach Branchen, 2016-2019. 43 


\section{Wirtschaftspolitisches Zentrum WPZ}

Forschung und Kommunikation auf Spitzenniveau für Politik, Wirtschaft und Gesellschaft

Das Wirtschaftspolitische Zentrum (WPZ) ist eine Initiative der Forschungsgemeinschaft für Nationalökonomie (FGN-HSG) an der Universität St. Gallen und ist folgenden Aufgaben gewidmet:

- Spitzenforschung mit Anwendungsbezug

- Wissenstransfer in die wirtschaftspolitische Praxis

- Förderung der wissenschaftlichen Nachwuchstalente

- Information der Öffentlichkeit

Unsere Aktivitäten in der Forschung reichen von wegweisenden Studien in Kooperation mit international führenden Wissenschaftlern bis hin $\mathrm{zu}$ fortlaufenden wirtschaftspolitischen Kommentaren. Damit wollen wir die wirtschaftspolitische Diskussion mit grundlegenden Denkanstößen beleben und eine konsequente Reformagenda für Österreich entwickeln, um die großen Herausforderungen besser zu lösen. Die Erkenntnisse und Ergebnisse der modernen Theorie und empirischen Forschung sollen zugänglich aufbereitet und kommuniziert werden, damit sie von Entscheidungsträgern und der Öffentlichkeit wahrgenommen und genutzt werden können und für die politische Entscheidungsfindung Relevanz entwickeln.

Wirtschaftspolitisches Zentrum | www.wpz-fgn.com | office@wpz-fgn.com 